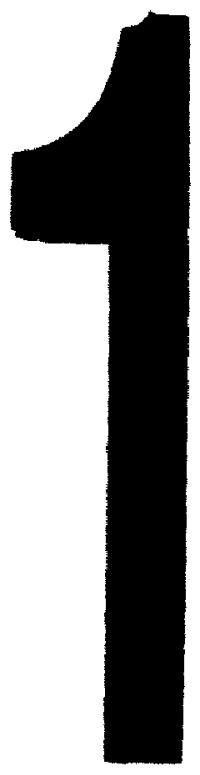

PM-1 3' $\times 4$ PHOTOGAAPHIC MICROCOPY TARGET NBS $1010 a$ ANSI/ISO \#2 EOUIVALENT

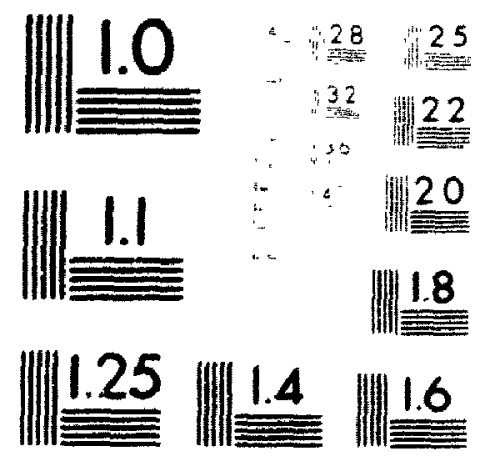

PRECISIO: SM RESOLUTION TARGETS 
The quality of this microform is heavily dependent upon the quality of the original thesis submitted for microfilming. Every effort has been made to ensure the highest quality of reproduction possible.

If pages are missing, contact the university which granted the degree.

Some pages may have indistinct print especially if the original pages were typed with a poor typewriter ribbon or if the university sent us an inferior photocopy.

Reproduction in full or in part of this microform is governed by the Canadian Copyright Act, R.S.C. 1970, c. C-30, and subsequent amendments.
La qualité de cette microforme dépend grandement de la qualité de la thèse soumise au microfilmage. Nous avons tout fait pour assurer une qualité supérieure de reproduction.

S'il manque des pages, veuillez communiquer avec l'université qui a conféré le grade.

La qualité d'impression de certaines pages peut laisser à désirer, surtout si les pages originales ont été dactylographićes à l'aide d'un ruban usé ou si l'université nous a fait parvenir une photocopie de qualité inférieure.

La reproduction, même partielle, de cet w microforme est soumise à la Loi canadienne sur le droit d'auteur, SRC 1970, c. C-30, et ses amendements subséquents. 


\title{
Characterization of Classes of Parallel Programs and Their Use in Performance Evaluation of Scheduling Policies
}

\author{
by \\ Terrence Siu-Lun Au, B. C. S. \\ A thesis submitted to \\ the Faculty of Graduate Studies and Research \\ in parial fulfillment of the requirements for the degree of \\ Master of Computer Science
Ottáwa-Carleton Insitute for Computer Science
School of Computer Scrence
Carleton University
Otawa, Ontario \\ Octoher 8, 1992 \\ copyright \\ 1992. Terrence Siu-Lun Au
}


The author has granted an irrevocable non-exclusive licence allowing the National Library of Canada to reproduce, loan, distribute or sell copies of his/her thesis by any means and in any form or format, making this thesis available to interested persons.
The author retains ownership of the copyright in his/her thesis. Neither the thesis nor substantial extracts from it may be printed or otherwise reproduced without his/her permission.
L'auteur a accordé une licence irrévocable et non exclusive permettant à la Bibliothèque nationale du Canada de reproduire, prêter, distribuer ou vendre des copies de sa thèse de quelque manière et sous quelque forme que ce soit pour mettre des exemplaires de cette thèse à la disposition des personnes intéressées.

L'auteur conserve la propriété du droit d'auteur qui protège sa thèse. Ni la thèse ni des extraits substantiels de celle-ci ne doivent être imprimés ou autrement reproduits sans son autorisation.

ISBN $\quad 0-315-84105-2$ 
The undersigned recommend to the Faculty of Graduate Studies and Research acceptance of the thesis

\section{"Characterization of Classes of Parallel Programs and Their Use in Performance Evaluation of Scheduling Policies"}

submitted by

Terrence S. L. Au, B.C.S.

in partial fulfillment of the requirements for the degree of Master of Computer Science

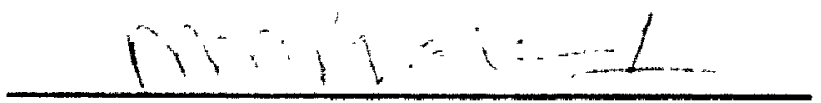

Thesis Supervisor

Chairman, School of Computer Science

Carleton University

October 8, 1992 


\begin{abstract}
Scheduling in multiprogrammed shared-memory multiprocessors has been studied for several years. Most of the previous studies modelled the workload by using a simple fork-and-join type of job structure. With this workload, the synchronization structure of parallel programs, which refers to the syrichronization constraints among the tasks of the programs, is not considered. However, the performance of scheduling policies could be affected by the presence of synchronization.
\end{abstract}

An abstract model is introduced tised on four different types of parallel programs: 1) fork-and-join, 2) divide-and-conquer, 3) Gaussian elimination and 4) state-space search with the possibility of premature task abortion. Each of these classes represents a different form of synchronization structure. Example programs from different application areas that belong to these classes are given to support the representativeness of the abstract model.

The performance of three scheduling policies, namely, coscheduling, round-robin job and dynamic partitioning are then studied with the new workload model to investigate how synchronization among tasks affects their performance. 


\section{ACKNOWLEDGEMENTS}

I would like to expiess my deepest appreciation to my thesis supervisor, Dr. S. Dandamudi, for his continued interest and guidance provided during this thesis and throughout my study at Carleton University. I would also like to thank Dr. G. White, University of Ottawa and Dr. S. Majumda, Dept. of System and Computer Engineering, for their comments in the final draft of the thesis.

In addition, I thank for the School of Computer Science for equipments and financial supports.

Finally, I would like to present this thesis to my beloved parents. 


\section{TABLE OF CONTENTS}

ACCEPTANCE SHEET

ABSTRACT

ACKNOWLEDGEMENTS iv

TABLE OF CONTENTS v v v

LIST OF FIGURES $\quad$ vii

LIST OF TABLES $\quad$ xi

CHAPTER I:

INTRODUCTION 3

1.1 Characterizing Parallel Programs 3

1.1.1 Sevcik's Models 3

1.1.2 Leutenegger's Models 4

2.1.3 Majumdar et al.'s Models 4

1.1.4 Ghosal et al.'s Models 5

1.1.5 Zahorjan and McCann's Models $\quad 6$

$\begin{array}{lll}1.2 & \text { Overview of The Thesis } & 8\end{array}$

\section{CHAPTER II:}

A SURVEY OF SCHEDULING POLICIES

2.1 Previous Works on Performance Evaluation of Scheduling $\begin{array}{ll}\text { Policies } & 10\end{array}$

$\begin{array}{lll}2.2 & \text { Motivation of The Thesis } & 17\end{array}$

CHAPTER III:

AN ABSTRACT MODEL OF PARALLEL PROGRAMS 18

3.1 Parallel Fork-and-Join Progrwass $\quad 18$

3.2 Parallel Divide-and-Conquer Programs 22

3.2.1 Implementing Divide-and-Conquer Algorithms in Parallel 23

3.2.2 Examples of the Parallel Implementation of Divide-and-

Conquer Algorithms 24

3.2.3 The Structure of Parallel Divide-and-Conquer 30

3.3 Parallel Matrix Factorization 31

3.3.1 Examples of Matrix Factorization Algorithm and Their Parallel Implementations $\quad 32$

3.3.2 The Structure of Parallel Matrix Factorization 43 
$\begin{array}{lll}3.4 & \text { Parallel Search } & 45\end{array}$

3.4.1 Breadth-first on A State-Space Graph 45

3.4.2 Implementing The Breadth-First Search in Parallel 50

3.5 Parameters of The Abstract Model 51

CHAPTER IV:

SCHEDULING POLICIES $\quad 55$

4.1 The CoScheduling Policy 56

4.2 The Preemptive Smallest Cumulative Demand First Policy 58

4.3 The Round Robin Job Policy 59

4.4 The Dynamic Policy 62

CHAPTER V:

SIMULATION EXPERIMENTS

5.1 The Impact of Job Models on the Performance of Scheduling Policies

5.1.1 Results of the Fork-and-Join Job Model 67

5.1.2 Results of the Divide-and-Conquer Job Model 70

5.1.3 Results of the Gaussian Elimination Job Model 72

5.1.4 Results of the Breadth First Search Job Model 73

5.1.5 Summary of the Results 75

5.2 Sensitivity of Performance to $C V_{d}$

5.3 Sensitivity of Performance to $n \quad 82$

5.3.1 Results of the Uncorrelated Workload 82

5.3.2 Results of the Correlated Workload 86

5.4 Impact of $C V_{n}$ on the Performance of Scheduling Policies 90

5.4.1 Discussion of Results for $\mathrm{CV}_{\mathrm{n}}=0.0$

5.4.2 Discussion of Results for $\mathrm{CV}_{\mathrm{n}}=5.0$

$\begin{array}{lll}5.5 & \text { Conclusions } & 106\end{array}$

CHAPTER VI:

CONCLUSION 107

$\begin{array}{lll}6.1 & \text { Summary } & 107\end{array}$

6.2 Contribution of the Thesis 107

$\begin{array}{lll}6.3 & \text { Further Research } & 108\end{array}$

$\begin{array}{lr}\text { REFERENCES } & 109\end{array}$ 


\section{LIST OF FIGURES}

Figure No.

1.1

1.2

1.3

2.1

3.1

3.2

3.3

3.4

3.5

3.6

3.7

3.8

3.9

3.10

3.11

3.12

3.13

3.14

3.15

3.16

3.17

3.18
Description

Page

The structure of parallel MVA programs

7

The structure of canonical fork-and-join programs

8

The structure of variable fork-and-join programs

8

Performance of the scheduling policies

14

A parallel root-search (or zero-search) program

19

A parallel n-body algorithm

The task graph of the parailel n-body

22

A schema for implementing divide-and-conquer in parallel

23

A paraliel implementation the Quicksort algorithm

25

A parallel implementation of the mergeson algorithm

26

An illustration of the quadrature problem

27

A parallel implementation of the adaptive quadrature algorithm

28

A parallel implementation of the convex hull algorithm

29

Illustration of the planar merge procedure

29

The task system of divide-and-conquer a jrithm

31

The LU Decomposition algorithm

An illustration of the reduction where $k=2$

34

The algorithm for $L U$ decomposition with partial pivoting

36

A parallel implementation of the $\mathrm{LU}$ decomposition algorithm

38

The Guass-Jordan algorithm

39

A parallel implementation of the Gauss-Jordan algorithm

40

The Cholesky factorization algorithm 
The configuration of the problem of changing three blocks

Performarce of scheduling policies (Cor,FJ)

Performance of scheduling policies (Cor,DC)

Sensitivity of performance to $C V_{d}$ (Cor, DC) 
Performance of scheduling policies when $C V_{n}=0.0$ (UnCor,FJ)

Performance of scheduling policies when $C V_{n}=0.0$ (Cor,DC)

Performance of scheduling policies when $C V_{n}=0.0$ (UnCor, GE) 96

Performance of scheduling policies when $C V_{n}=0.0$ (UnCor,SSS) 98

Performance of scheduling policies when $C V_{n}=5.0(\mathrm{Cor}, \mathrm{FJ})$

Performance of scheduling policies when $C V_{n}=5.0$ (Cor,DC)

Performance of scheduling policies when $C V_{n}=5.0$ (UnCor,DC)

Performance of scheduling policies when $C V_{n}=5.0(\mathrm{Cor}, \mathrm{GE})$

Performance of scheduling policies when $C V_{n}=5.0$ (UnCor,GE) 103 


\section{LIST OF TABLES}

Table No.

2.1

5.1

5.2

5.4

5.5

5.6

5.8

5.9
Description

Page

Performance of Scheduling Policies

17

The output parameters and their descriptions

64

Default values of the input parameters (uncorrelated workload)

(Impact of Job models on the performance of scheduling policies)

Default values of the input parameters (corelated workload)

(Impact of Job models on the performance of scheduling policies)

Default values of the input parameters (uncorrelated workload) (Sensitivity of the Performance $C V_{d}$ )

Default values of the input parameters (comelated workload)

(Sensitivity of the Performance $C V_{d}$ )

Default values of the input parameters (uncorrelated workload) (Sensitivity of Performance to $\mathrm{n}$ )

Default values of the input parameters (correlated workload)

(Sensitivity of Performance to $\mathrm{n}$ )

Default values of the input parameters (uncorrelated workload) (Impact of $C V_{n}$ on the performance of the scheduling policies)

Default values of the input parameters (correlated workload)

(Impact of $C V_{n}$ on the performance of the scheduling policies) 


\section{CHAPTER I}

\section{INTRODUC TION}

Advances in computing technology and the demands for faster computing systems have led to the development of different parallel processing systems. In these parallei systems, multiple processors work cooperatively on a parallel solution to a problem. The processors communicate through an interconnection network. The computation of a parallel job (or program) is divided into small chunks, each of which may potentially be executed in parallel. These chunks of a parallel computation are called tasks. Task granularity is an important issue in determining the performance of parallel systems At one extreme, the entire job can be considered as a single task and at the other extreme we can consider each executable instruction as a task. Fine task granularity allows maximum exploitation of the parallelism that is inherently present in a program. The dataflow architecture is an example that supports task granularity at the instruction level. However, the overheads associated with the management of a large number of small tasks may cause implementation and performance problems. This thesis concenurates on systems that support medium grain size. These systems include ihe shared-memory parallel systems like the Sequent Symmetry [Lovett \& Thakker 88], Deconler HEP [Simth 78] and the Stanford Dash [Lenoski et al. 92].

In a parallel system, perfect speed-up is not always achievable. A poor operating system design and synchronization among tasks are some of the major factors affecting the performance. In particular, the scheduling of tasks to run on multiple processors, an important mechanism of the operating system, directly affects the performance. As a 
consequence, scheduling in multiprocessor systems has received considerable attention in the last decade.

Many approaches can be used to study the performance of scheduling algorithms. If the hardware and software are available at hand, we can implement the scheduling algorithms and use benchmarks to evaluate their performance. $A$ benchmark is a complete program that is written in a high level language and is considered to be the representative of a given class of application programs [Kobayashi 81]. The major problem with this approach is that the results obtained are too ppecific and they can only be applied to that particular type of parallel machine. Annther method is to use an abstract model to represent both the hardware and software. The abstract models can purely be mathematical where they can be formulated by using queuing theory. Such abstract model is called an analytical model. The values of the performance measures of an analytical model can be computed directly. However, the systems to be studied are of ten too complicated and their corresponding anaiytical models are not mathematically tractable [Kobayashi 81]. Highlevel simulation is the best alternative to the above techniques. There are two types of simulation: trace-driven and self-driven. Trace-driven simulation, as indicated by the name, refers to the use of traces as the inputs of the simulation program. A trace is a stream of events observed in an operational system. The problem of trace-driven simulation is that the traces of a computer system may not be available. In self-driven simulations, the inputs of the simulation programs are represented by a set of parameters and their values are generated from probability functions with appropriate statistical distributions. Identifying the set of parameters that represents the actual input sequences is important in performing the self-driven simulation. 


\subsection{Characterizing Parallel Programs}

In this section, the methods to represent the purallel programs are discussed. Parallel programs can be characterized at a number of different levels of details [Sevcik 89]. At a higher level, they can be characterized by a single parameter. For example, the maximum parallelism or the maximum number of tasks that can be run in parallel can be used. At a lower level, the data dependency graphs can be used to characterize the parallel programs. High level characterization is not usefui for performance evaluation, as a lot of information on parallel programs will be overlooked. For example, if the abstract model consists of a single parameter, i.e., the maximum number of tasks, it cannot be used to describe the synchronization among tasks. However, low level characterization may also be inappropriatc because of the high cost of managing huge amounts of information. In the following sub-sections, a survey different approaches to characterize parallel programs is given.

\subsubsection{Sevcik's Models}

A number of parameters for characterizing parallel programs is defined and their use in decision making for processor allocation is discussed in [Sevcik 89]. The first parameter is the average parallelism $(A)$, defined as the average number of busy processors during the execution of the application when an unlimited number of processors are available. It can also be defined as the ratio of the time to execute on a single processor to the time on an unlimited number of processors. The minimum parallelism $(m)$ and maximum parallelism $(M)$ of an application, are defined as the minimum and maximum number of busy processors during the execution of the application. The last parameter is the variance of parallelism ( $V$ ) of an application, which represents the variance of the number of busy processors when the application is being executed, when there is no limitation on the 
processing resources. It should be noted that Sevcik made use of these parameters to improve the performance of static scheduling in multiprocessor systems.

\subsubsection{Leutenegger's Models}

A relatively high level characterization of fork-and-join parallel programs ${ }^{1}$ is presented in [Leutenegger 90]. In their studies, parallel programs are characterized by two parameters, the number of processes and service demand of parallel jobs. The service demand is the execution time of all the processes that a parallel program has. In addition. they assume that the service demand is distributed uniformly among all the processes of the job. Various statistical distributions are used to compute values for these two parameters.

\subsubsection{Majumda: et al.'s Models}

Another high level characterization of parallel programs is given in [Majumdar et al. 91]. Two parameters are introduced for the characterization. The first one is the average parallelism $(A)$ of a job, defined as the average number of processors that are busy during its execution given an unbounded number of available processors. Another parameter is the execution time of jobs with A processors, $\omega(A)$, defined as the execution time attained by a job with A processors when the processors allocated to the job are shared equally by the currently active tasks in the job. The $\omega(\mathrm{A})$ is a measure of the variation in the job's parallelism. A high value of $\omega(A)$ represents a large variation in the parallelism. These two parameters together with the arrival rate form a characterization of parallel programs.

For a given value of A, four structures of parallel programs are used. Each of these structures is represented by a value of $\omega(A)$. The values of $\omega(A)$ of these structures and their descriptions are given in Table 1.1. Different workload models are derived by varying

\footnotetext{
1 The meaning of fork-and-join parallel programs will be given in chapter.
} 
the values of these parameters. The workload models are empioyed to study the performance of static and semi-static scheduling policies.

\begin{tabular}{|c|c|}
\hline$\omega(A)$ & Description \\
\hline 1 & A single phase with parallelism A \\
\hline $2-\frac{1}{A}$ & $\begin{array}{l}\text { A sequential phase followed by a phase with unbounded } \\
\text { parallelism }\end{array}$ \\
\hline $1.5-\frac{1}{2 A}$ & $\begin{array}{l}\text { A sequential phase followed by a phase with parallelism } \\
2 \mathrm{~A}-1\end{array}$ \\
\hline 1.5 & $\begin{array}{l}\text { A phase with parallelism of } \frac{A}{2} \text { and a phase of unbounded } \\
\text { parallelism }\end{array}$ \\
\hline
\end{tabular}

Table 1.1 The values of $\omega(A)$ and their descriptions [Majumdar et al. 91]

\subsubsection{Ghosal et al.'s Models}

A single parameter for characterizing the behaviour of parallel programs is given in [Ghosal et al. 91]. This parameter is called the processor working set (pws).

A number of parameters are defined first, in order give a definition for $p$ w's. The first parameter is the aggregate execution time of a program $T(p)$ which is the sum of $T_{\text {comp }}(p)$ and $T_{\text {comm }}(p) . T_{\text {comp }}(p)$ represents the time for performing actual computation of the program and $T_{\text {comm }}(p)$ is the overhead in the program. Then the efficacy of a parallel program is given by $\eta(p)=\frac{S^{2}(p)}{p}$, where $S(p)=\frac{T(1)}{T(p)}$ is the speedup of the program given p processors.

Then, the parameter $p w s$ is defined as the minimum number of processors that achieves the absolute maximum of $\eta(p)$.

In [Ghosal et al. 91], the parameter pws is used to study the performance of four programs. Test runs of the programs on a transputer network are conducted to obtain 
values for the parameters $T_{\text {comp }}(p)$ and $T_{\text {comm }}(p)$. The values for the efficacy is derived to study the performance of scheduling for parallel systems.

\subsubsection{Zahorjan and McCann's Models}

A method that makes use of the concept of benchmarking and synthetic programs are used in [Zahorjan \& McCann 90]. Three classes of benchmark parallel programs are first selected. The task graph and execution time distribution are then derived for each class of parallel programs. Finally, a special parameter is derived for each class and a statistical distribution is used to generate values for the parameter so that different instances of the parallel programs can be generated. Zahorjan and McCann's approach is considered to be a low-level characterization of parallel programs.

An example of the first class is the parallel Mean Value Analysis solution package (MVA) for product form queuing networks containing two classes of $N$ customers each. This is a parallel implementation of the dynamic programming [Horowitz \& Sahni 78], commonly used to solve problems where their solutions may be viewed as the result of a sequence of decisions ${ }^{2}$. This parallel program exhibits a wave-front computational structure, in a way that the number of tasks in the program changes according to the sequence $1,2,3, \ldots, N, N+1, N, \ldots, 3,2,1$ during its execution. The computational structure of this program where $N$ is equal to 5 is depicted in Figure 1.1. The parameter associated with this class of abstract models is the number of customers $N$.

The second class of parallel programs is called the canonical fork-and-join. These parallel programs cycle through between sequential phases and phases with $K$ runnable tasks. The parameter associated with this class of abstract models is the number of nunnable

\footnotetext{
2 The problem of finding a shortest path from vertex $i$ to vertex $j$ in a directed graph $G$ is another example of problems of this type. The problem is to decide the sequence of verices that are to be traveled from $i$ to $j$. where the path from $i$ to $j$ has minimum length.
} 
tasks $\boldsymbol{K}$. An example program of this type is a parallel implementation of the matrix multiplication given in [McCann et al. 90]. The matrices to be multiplied are divided into fixed-size blocks, and partial results are computed for the corresponding blocks in the result matrix. The parallel computation is done by creating tasks for each block in the result matrix. The structure of these programs is shown in Figure 1.2.

The third class of parallel programs is the variable fork-and-join. The task graph of this algorithm is similar to the canonical fork-and-join, that is, repeated cycles of sequential and parallel phases. The only difference is that the number of tasks is varie $\lrcorner$ in each parallel phase. The number of tasks to be created at each parallel phase is decided based on the number of processors available. The structure of these programs is shown in Figure 1.3.

These three classes of parallel program models are employed to study the performance of static and dynamic scheduling policies.

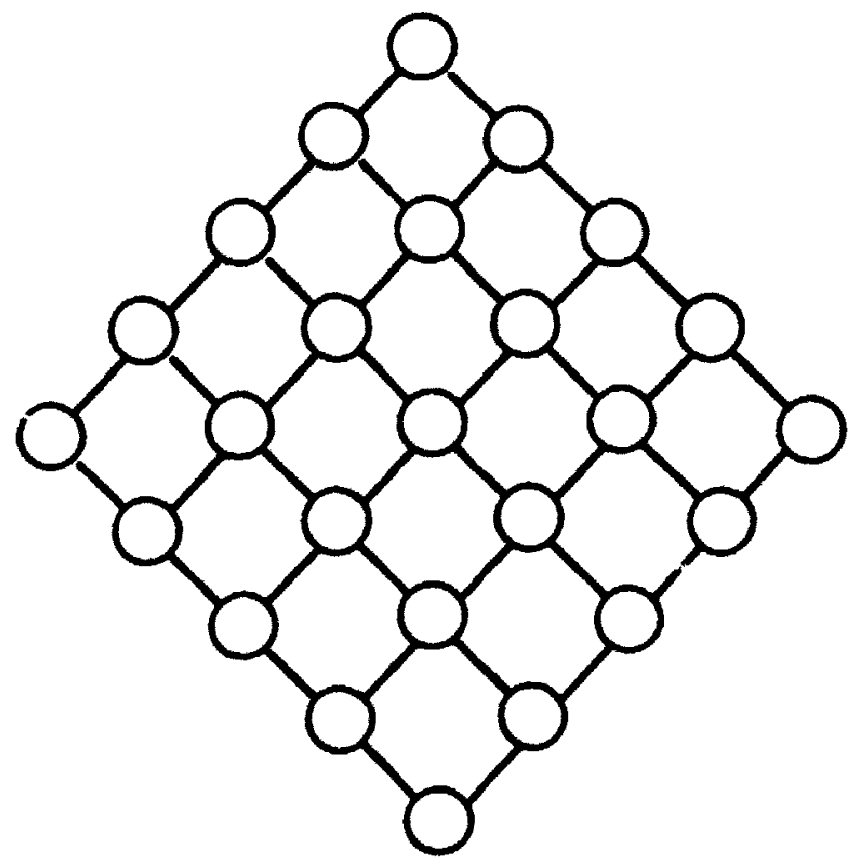

Figure 1.1 The structure of parallel MVA programs [Zahorjan \& McCann 90] 


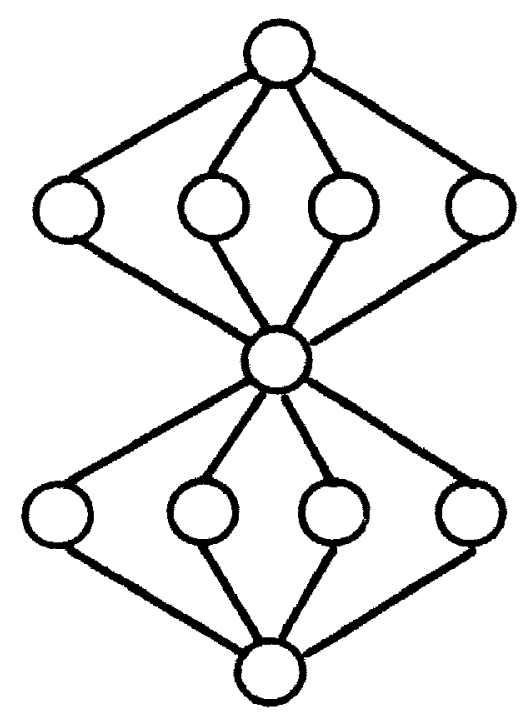

Figure 1.2 The structure of canonical fork-and-join programs [Zahorjan \& McCann 90]

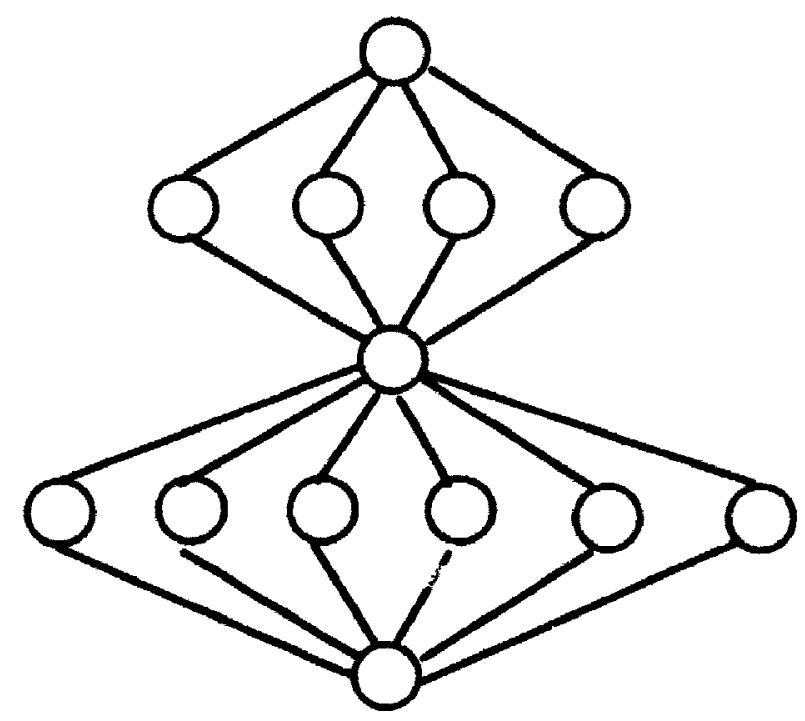

Figure 1.3 The structure of variable fork-and-join programs [Zahorjan \& McCann 90]

\subsection{Overview of The Thesis}

The remainder of the thesis is organized as follows.

Chapter 2 gives a survey of scheduling policies for multiprogrammed shared memory multiprocessors. At the end of this chapter, we review the problems associated with the workload models in the previous studies to provide motivation for this thesis. 
Chapter 3 gives a detailed description of the parallel implementation of four classes of algorithms. We then derive a parallel job model based on these parallel programs. This job model will be used to evaluate the performance of four scheduling policies.

Chapter 4 presents a detailed description of the selected scheduling policies. We also discuss the rationale for choosing these scheduling policies.

Chapter 5 presents the simulation results together with sensitivity analysis of the parameters of the parallel job model. We compare our results to the previous ones to see whether our new job model affects the performance of the scheduling policies.

Finally, Chapter 6 provides a summary of the thesis. The contributions of the thesis and directions for further research are also given. 


\section{CHAPTER II}

\section{A SURVEY OF SCHEDULING POLICIES}

In this chapter, we revien previous work in the area of processor scheduling for multiprogrammed shared-memory multiprocessors.

\subsection{Previous Work on Performance Evaluation of Scheduling Policies}

In this section, we give a brief introduction to the scheduling policies that have been studied in the literature. The scheduling policies are 1) the first-come-first-served policy $F C F S, 2)$ the smallest number of tasks first policy SNTF, 3) the smallest cumulative demand first policy $S C D F, 4)$ the task-based round robin policy RRtask, 5) the job-based round robin policy $R R j o b, 6$ ) the dynamic partitioning policy $D P$ and 7) the coscheduling policy Cosched. The scheduling policies can be divided into three categories, 1) Run-toCompletion, 2) Preemptive and 3) Dynamic.

\section{Run-to-Completion Scheduling}

Three run-to-completion scheduling policies have been considered extensively. By run-to-completion, we mean that when a processor is assigned to execute a task, it continues the execution uninterrupted until the termination. Each of the three policies has its own queuing discipline, the logical ordering of tasks in a queve and determines which task will be chosen for service when a server becomes free. One policy orders the tasks queue on a first come first served basis. The priority depends on some properties of the parallel jobs. These policies are described as follows. 
- First Come First Served $(F C F S)$ : When a parallel job arrives, its tasks are stored at the end of the queue. If there is a free processor available, a task at the head of the queue is scheduled.

- Smallest Number of Tasks First (SNTF) : The queue is ordered on a priority basis with the highest priority given to the tasks of the paraliel job that has the smallest number of tasks. When two jobs have the same number of tasks, the one that arrives earlier gets a higher priority. When a processor becomes idle, the first task from the queue is scheduled.

- Smallest Cumulative Demand First (SCDF) : The queue is ordered on a priority basis with the highest priority given to the tasks of the parallel job which has the smallest cumulative demand. The cumulative demand is the sum of the service times of a job's tasks. When two jobs have the same cumulative demand, the one that arrives earlier gets a higher priority. When a processor becomes idle, the first task from the queve is scheduled.

\section{Preemplive Scheduling}

The run-to-completion scheduling policies have the problem that when a task is assigned to a processor, it must be executed until its termination. Consider the SCDF policy. At time 0 , there is one job on the queue with 4 units of service demand. We assume that all processors are used by this job. Later on, say at time 1 , a new job comes with 1 unit of service demand. The new job will only be served after the first job terminates, even though the new job has smaller service demand than the old job.

To solve this problem, the system can deschedule some tasks of the running jobs so that other jobs can be served. This is called the preemption of tasks from processors. There are two types of preemptive scheduling policies: variable and fixed. 
For variable preemptive scheduling, the processor reallccation only occurs when a new joh arrives or an old job terminates. We consider the variable preemptive scheduling policies with SNTF and SCDF queuing discipline.

- Preemptive Smallest Number of Tasks First (PSNTF): This is a variation of the SNTF. A priority is assigned to each job on the queue. The priority value depends on the number of tasks in the parallel jobs. The decision of preempting tasks from the processors is done by comparing the priority (number of tasks) of the new job to the jobs that are currently being executed.

- Preemptive Smallest Cumulative Demand First $(P S C D F)$ : This is a variation of the $S C D F$. The task queue is organized on a priority basis. The priority value of the jobs depends on the remaining cumulative demand associated with them. A newly arrived job can preempt processors from the tasks that have lower priority (smaller cumulative service demand).

\section{Round-Robin Scheduling}

In a round-robin policy, services occur in increments of some parameter $\alpha$, say $\alpha=$ 2 time units. When a task gets to the head of the queue, the task receives $\alpha=2$ time units of service. After being served for $\alpha=2$ time units, the task is descheduled from the processor and placed back in the queue. The parameter $\alpha$ is called a quantum. Three variations of the round-robin scheduling have been studied intensively. They are described as follows.

- Task-based Round robin (RRtask) : When a job arrives, it is divided into a set of tasks that are placed at the end of the shared task queue. The round robin scheduling is applied to the task queue. 
- Job-based Round robin (RRjob) : This policy is similar to the Task-based Round robin policy but instead of sharing a task queve, there is a shared job queve. Each entry in the job queue has a queue maintaining its own tasks. Scheduling is done round robin on the parallel jobs. The job at the front of a queue receives $P$ quanta of size $q$. where $P$ equals to the number of processors in the system.

- Coscheduling (Cosched) : The queuing discipline is like the one for the task-based round robin policy. Scheduling is done by moving a window of length equal to the number of processors in the sys'em along the task queue. Each task in the window gets one quantum of service on a processor. At the end of the quantum, the window is moved down the queue until the first slot of the window is over the first task of a job that was not scheduled in the previous quantum. The quantum completes for each processor at the same time.

\section{Dynamic Scheduling}

Under the dynamic scheduling policies, processors are realiocated among jobs in response to changes in the jobs' parallelism, arrival or termination of parallel jobs. The goal of this scheduling policy is to allocate processors to parallel jobs as evenly as possible. Processors may be preemptor from one job to another, if it is required. The policy is called dynamic partitioning as it partitions the processors among the jobs in the system.

- Dynamic Partitioning (DP) : When a job arrives, the following actions are taken: 1 ) if there are idle processors allocate them to the job. If the job needs fewer processors than the free processors, just allocate as many as are needed. 2) If there is no idle processor and there is a job that holds more than one processors, a processor is preempted from this jeb and is allocated to the new job, 3) If no processor is idle and no job has two or more processors, the new job must wait until a schedulcd job departs the system. 
When a task finishes, the following actions are taken: 1) if the job has other tasks still not scheduled, schedule one of them on the processor; 2) if the job has no unscheduled tasks allocate the processor to the first job that has unallocated tasks. based on their arrival time.

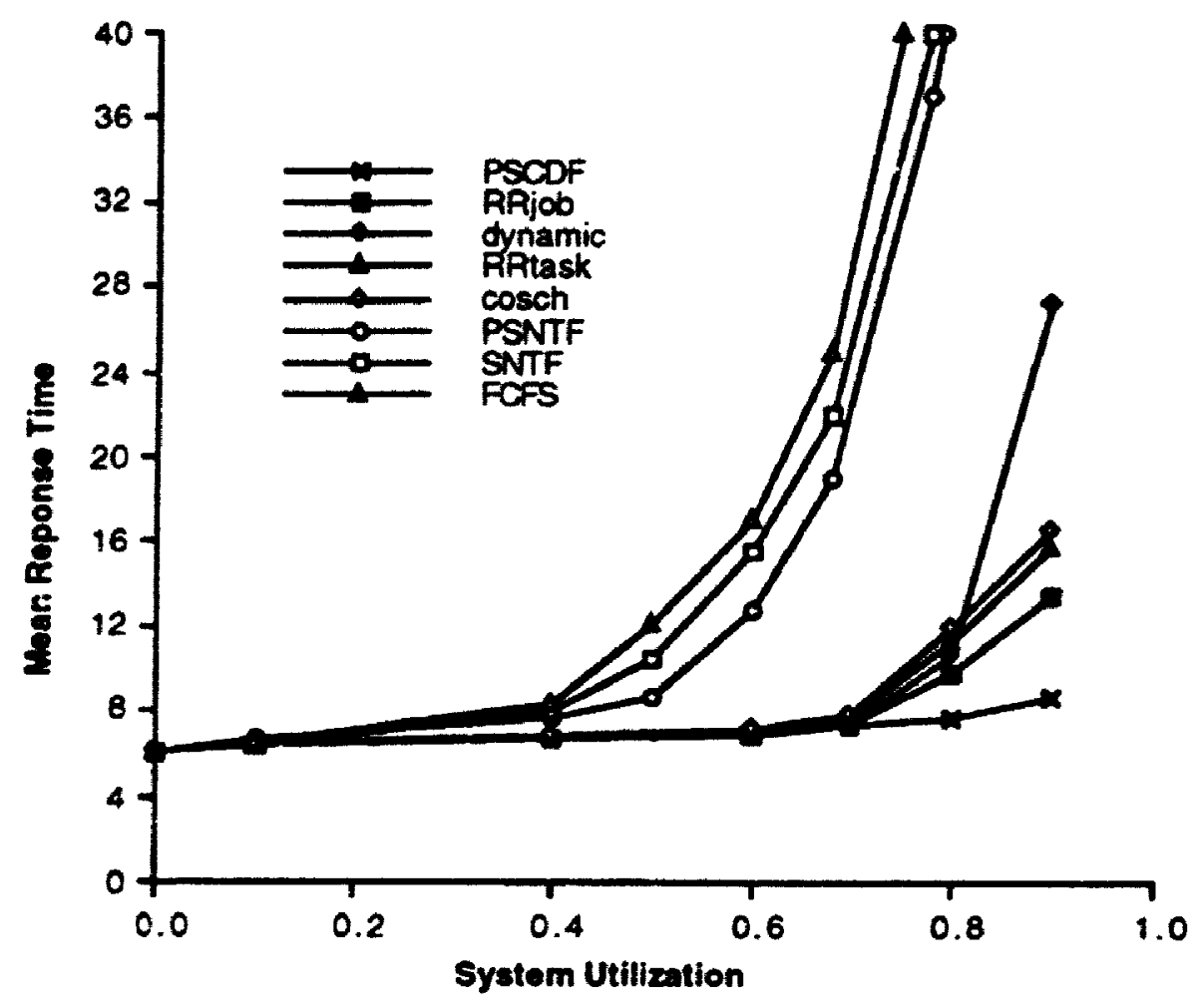

Figure 2.1 Performance of the scheduling policies [Leutenegger and Vernon 90]

Figure 2.1 shows the result of an experiment conducted by Leutenegger and Vernon that illustrates the performance of the policies. In Leutenegger and Vernon's paper. processes are assumed to be the smallest schedulabie unit and a process may execute several tasks serially. In our case, we assume that tasks are the smallest schedulable units and there is a single queue of runnable tasks in the system. We will discuss their results based on some assumptions: 1) a parallel job is a collection of independent tasks and there 
is no communication among tasks. 2) parallel jobs are modeled by two parameters, number of tasks and service demand and 3) there is a positive correlation between these parameters. Therefore, jobs with large service demand always have large number of tasks.

\section{Resulis of the nu-to-completion scheduling}

- First Come First Served (FCFS) : FCFS is the worst policy as shown in Figure 2.1. The major problem of FCFS is that it allows jobs with large service demand to monopolize the processing power, making the smaller jobs wait unnecessarily. A longer waiting time results in a longer response time.

- Smallest Number of Tasks First (SNTF) : SNTF performs poorly and it is the second worst policy. SNTF suffers from the same problem as the $F C F S$, by permitting large jobs to dominate over the smaller ones.

\section{Results of variable preemntive scheduling}

- Preemptive Smallest Number of Tasks First (PSNTF) : PSNTF performs poorly, even though preemption is used. This is because the number of tasks in a parallel job does not give accurate information on the size of a parallel job. That is a large job can have small number of tasks with large service demand for each task of the job. Hence, this policy performs similar to the FCFS and SNTF policies.

- Preemptive Smallest Cumulative Service Demand First $(P S C D F)$ : it is the best scheduling policy. Since the cumulative service demand is used for determining which tasks are to be scheduled, the processing power of the system can be distributed equally among the parallel jobs. 


\section{Results of the round robin policies}

In general, the round robin policies perform hetter than the staiic ânu the preemptive static policies, except for the PSCDF policy. In a round robin policy. if both the task-based and joh-based, is applied, the effect of large jobs dominating over the smaller ones can be reduced. The result of each policies is discussed below.

- Tasks-based Round Robin (RRtask) and Coscheduling (Cosched) : Since RRtask and Cosched perform similarly. we will discuss the result of these policies together. Note that Cosched performs slightly worse than RRtask which implies that it is not worth to have coscheduling without process communication. Due to the ability to time-slice the processing power among the jobs in the system, the effect $u$. the large jobs can be reduced. As a result, $R R t a s k$ performs better than the non-preemptive policies, like FCFS and SNTF. However, the allocation of processing power is still unfair, as large jobs can still dominate if the number of tasks is correlated to the size of the jobs. Hence, the johs with large number of tasks may monopolize the system leading to a performance degradation. This problem is overcome by the job-hased Round Robin policy.

- Job-hased Round Rohin (RRjob) : Since the policy varies the quantum size according to the number of tasks in a job. the allocation of processing power does not depend on the joh size. The improvement makes RRjob the best in the group round robin policies.

\section{Results of Dynamic Scheduling}

- Dynamic Partitioning (DP) : Since $D P$ can vary the number of processors allocated to parallel jubs according to the number of executable tasks in the job, the dynamic partitioning performs well. Its performance is comparable to that of the RRjob policy. However, this policy performs poorly when the system load is at around 90\%, hecause 
the number of parallel jobs exceeds the number of processors in the system which results in a large number of jobs waiting in the queue.

The performance results of the above algorithms is summarized in Table 2.1. We assign a rank to each policy and order the table according to their rank.

\begin{tabular}{|c|c|c|}
\hline Scheduling Policies & Categories & Rank \\
\hline PSCDF & Preemptive (fixed) & 1 \\
\hline RRjob & Preemptive (variable) & 2 \\
\hline$D P$ & Dynamic & 3 \\
\hline RRtask & Preemptive (variable) & 4 \\
\hline Cosched & Preemptive (variable) & 5 \\
\hline PSNTF & Preemptive (fixed) & 6 \\
\hline SNTF & Run-to-completion & 7 \\
\hline FCFS & Run-to-completion & 8 \\
\hline
\end{tabular}

Table 2.1 Performance of Scheduling Policies

\subsection{Motivation for The Thesis}

It can be seen from the review of the models that many of them cannot be used to specify the synchronization structures of parallel jobs. Some exceptions are [Zahorjan \& McCann 90] and [Majumdar et al. 91], where some forms of synchronization structuies are considered. Synchronization is a fundamental issue in parallel processing and it is not clear whether changing from one synchronization structure to another would affect the performance of scheduling policies. In the thesis, four classes of parallel programs are considered, each of which represents a synchronization structure. The classes of parallel programs include, the fork-and-join, divide-and-conquer, gaussian elimination and statespace search. These classes will be discussed in Chapter 3 . The objective of the thesis is to investigate the effects of changing the synchronization structures on the performance of multiprocessor scheduling. 


\section{CHAPTER III}

\section{AN ABSTRACT MODEL OF PARALLEL PROGRAMS}

In this chapter, a survey of some parallel implementations of algorithms for sharedmemory multiprocessors is presented. The structure of these parallel programs is discussed. Then an abstract model of parallel programs that is based on the structure and other characteristics of the parallel programs surveyed is proposed. The abstract model is used to drive the simulation experiments to compare performance of scheduling algorithms. The remainder of this chapter is organized as follows. The next four sections, present various parallel implementations of algorithms. The abstract model is then presented in Section 3.6.

\subsection{Parallel Fork-ard-Join Programs}

In this section, the algorithms which can be decomposed into independent subcomputations are :onsidered. A simple example is the algorithms that consist of a single loop and each instance of the loop can be executed independently. The parallel implementation of these types of algorithms can be done by creating tasks to work on the sub-computations.

\section{Examrie 1: Ront-Search}

Given a continues function $f$ having opposite signs at the end-points of an interval of length $L$, locate a zero of the function $f$ within a unit interval. The parallel implementation of this algorithm is proposed in [Akl 89] and [Kung 76]. The interval to be evaluated is divided into $N$ sub-intervals and are assigned to the tasks. Each task attempts to find a root within their assigned sub-interval by using the Newton method. The tasks are 
synchronized when all of them finish and a root is found. The parallel program is shown in Figure 3.1. Since $f(x), f^{\prime}(x)$, and $f^{\prime \prime}(x)$ are continuous and bounded on each interval ( $a$, b), at least one interval will embrace a root of the function $f$.

PROCEDURE Root_Search $(f, f, a, b, c, r$, ROOT )

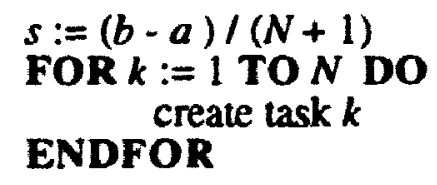

\section{TASK $k$}

Xold : $=\mathbf{a}+k s$

iteration : $=0$

WHILE (iteration $<r$ ) AND (ROOT $=\infty$ ) DO iteration := iteration +1

Xnew $:=$ Xold $-f($ Xold $) / f$ (Xold )

IF abs (Xnew - Xold ) <c THEN ROOT := Xnew

END WHILE

\section{END TASK $k$}

\section{END PROCEDURE}

Figure 3.1 A parallel root-search (or zero-search) program

\section{Example 2: The Parallel n-hodv Algorithm}

Another example algorithm with the fork-and-join structure is the parallel $n$-body algorithm given in [Almasi \& Gottlieb 89]. The basic problem can be stated as follows: given a collection interaction energy between bodies, predict the change in the interaction energy of the bodies over a period of time. These bodies are considered to be spherical with center-of-mass coordinate vectors $r_{1}, r_{2}, \ldots, r_{n}$.

We define the interaction energy between two bodies to be

$$
E_{i j}(r)=B_{i}\left(r^{*} / r\right)^{9}+z_{i j} z_{j} B_{j} / r
$$


where $z_{i}= \pm 1$ is the charge on ion $i$, the parameters $B_{1}, r^{*}$, and $B_{2}$ are special constants and $r=r_{i}-r_{j}$. And the binding energy of one of the bodies is defined as

$$
B_{i}=\sum_{j \neq i} E_{i j}
$$

It is assumed that the initial configuration and values of the initial energy are available. The bodies are moved randomly to generate a Markov chain of configurations. In this algorithm, the bodies are moved one at a time and the energy of the system atter a move is given in terms of the energy prior to the move by

$$
E_{i}^{\text {new }}=E_{i}^{\text {old }}+B_{i}^{\text {new }}-B_{i}^{\text {old }} \cdot 1
$$

The bottleneck of the algorithm involves the computations required to calculate the binding energy of the moved body, following and preceding its actual move. This computation of the binding energy involves evaluating the interaction of the moved body with each of the other bodies. However, the interactions can be evaluated in parallel with no communication between the computation. The algorithm is shown in Figure 3.2. In Figure 3.2, the function barriersync() specifies the points where parallel tasks perform their synchronization. The structure of this program is shown in Figure 3.3.

PROCEDURE n-body ()

WHILE (1)

select a body to be moved

$$
\text { FOR } \mathrm{j}=1 \text { TO } \mathrm{n} \text { DO }
$$

$1 \mathrm{i}$ is the moved body.

$E_{i}^{\text {old }}\left(E_{i}^{\text {new }}\right)$ is a vector of interactuon energy between the moved bxdy $i$ and all the other bodies before (after) the move.

$B_{1}^{\text {new }}\left(B_{1}^{\text {Old }}\right.$ ) is the binding energy of $i$ before (after) the nove. 


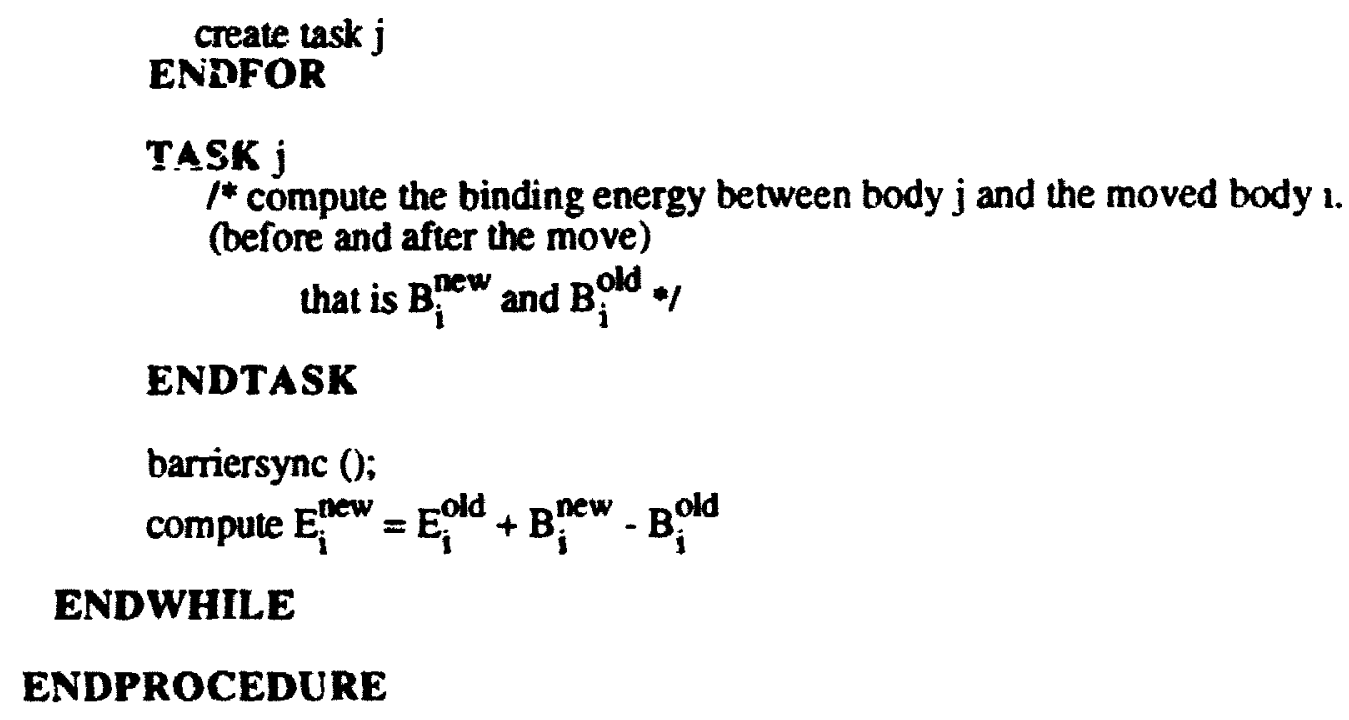

\section{ENDWHILE}

\section{ENDPROCEDURE}

Figure 3.2 A parallel n-body algorithm

\section{Example 3: A Parallel Event Driven Simulator}

Another example of the fork-and-join structure is a parallel, distributed time, event driven simulator for verifying the behaviour of a logic circuit. This simulator is based on a disuributed-time algorithm given in [Chandy \& Misra 81].

In this simulator, a logic circuit is modeled by a collection of elements, represented by its inputs, outputs and internal state. These elements are interconnected by wires. An element can be any functional block in the circuit. It can be as simple as a two input gates such as AND-gate or OR-gate or it can be as complicated as a CPU. The goal of performing logic circuit simulation is to test the circuit by feeding different inputs and see whether the circuit has the expected output behaviour. An event in the simulation corresponds to a computation of the new output behaviors of a gate by feeding in the given inputs.

Discrete event simulation is inherently sequential because of the presence of time ordering. However, it can be parallelized by having the elements in the circuit maintain their own time independently of other elements. Different elements might have different notions 
of the current simulated time. If something occurs at each of the inputs of an element, it will receive a time-stamped event on its outputs. In actual implementation, an element is activated for evaluation when it receives a new event. An activation of an element will generate a task that computes the outputs hehaviour of the element. In [Singh et al. 91], the algorithm is implemented in a way that allows the possibility of deadlocks signaled by having no task on the queue. The computation of tasks can be done independently. If a deadlock occurs, the algorithm will resolve it and continue the execution as usual. The parallelism in the algorithm reduces to 1 , when a deadlock occurs. The structure of this parallel program is the same as the one for the parallel n-body program shown in Figure

\section{3 .}

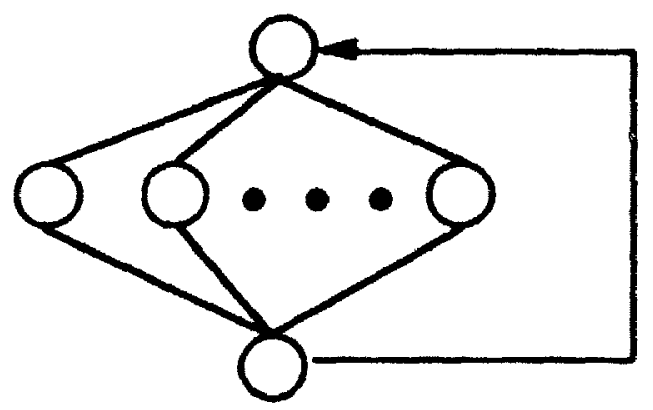

Figure 3.3 The task graph of the parallel n-body

\subsection{Parallel Divide-and-Conquer Programs}

In this section, a parallel implementation of the divide-and-conquer algorithms is considered. Large numbers of parallel programs have been developed that are based on this strategy, especially from the area of symbolic computation, where the computation works on a discrete data structure. In general, the divide-and-conquer strategy does not restrict the number of sub-problems into which a given problem is partitioned. However, we will restrict ourselves into the binary case, where a given problem will only be divided into two 
subproblems. Thus, the parallelism in these programs steps through the sequence $2^{0}, 2^{1}$, . $., 2^{n-1}, 2^{n}, 2^{n-1}, \ldots, 2^{1}, 2^{0}$ during their execution. Efficient parallel implementations of divide-and-conquer algorithms have been developed in many areas of application, such as, database management [Bitton et al. 83], [Deminet 82], [Evans \& Yousif 85], numerical integration [Grit \& Mcgraw 85], computational geometry [Knecht 90] and VLSI circuit design [Hachtel \& Moceyunas 88] and combinatorial problems [Knecht 89]. [Shekhawat \& Chakravarty 90]. We will consider the implementation of a subset of these algorithms.

\subsubsection{Implementing Divide-and-Conquer Algorithms in Parallel}

An implementation of the divide-and-conquer algorithms on parallel systems has received significant attention in the last decade. One way to parallelize the divide-andconquer algorithm is to use the COBEGIN/COEND construct. This implementation is given in [Horowitz \& Zorat 83], where a schema of the parallel implementation of divideand-conquer algorithms is presented. A reproduction of the schema is shown in Figure 3.4.

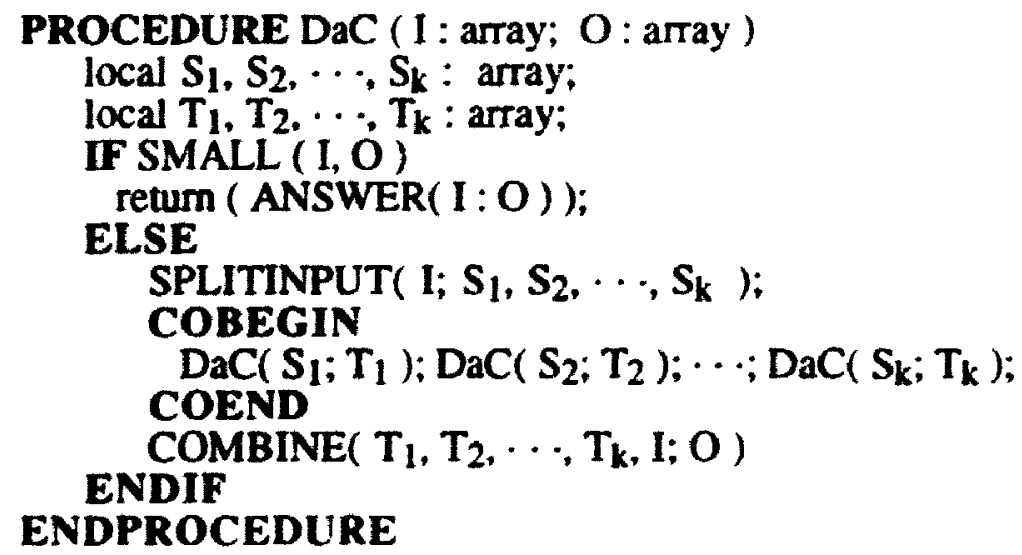

Figure 3.4 A schema for implementing divide-and-conquer in parallel [Horowitz \& Zorat 83]

The function SMALL determines whether further partition of the array I is needed. The function SPLITINPUT generates $k$ input arrays for the next recursive step based on the original input $l$. Within the COBEGIN/COEND, $k$ independent tasks are created and 
executed simultaneously. The function COMBINE groups together the outputs of the recursive calls and produces the output.

When the above program is executed, $k$ independent inputs $S_{1}, S_{2}, \cdots, S_{k}$ are generated from the original input $I$. Thus, there is no data dependence among tasks that work on the input $S_{1}, S_{2}, \cdots, S_{k}$. The data for $S_{1}, S_{2}, \cdots, S_{k}$ can either consist of one (or more) unmodified copy (copies) of the data in I, partitioned among $k$ structures, or it may consist of data that is actually computed from the data in $I$. The above program represents a general divide-and-conquer where $k$ recursive calls will be made at each level. However, we are only interested in the binary case where there are at most only two recursive calls at each level.

\subsubsection{Examples of the Parallel Implementation of Divide-and-Conquer Algorithms}

In this section, we present some example parallel implementations of divide-andconquer algorithms on multiprocessors.

\section{Example 1: Parallel OuickSort}

The first one to be discussed is the quick sort given in [Deminet 82], [Evans \& Yousif 85]. It first creates tasks to partition the input array using a median element, then generates more tasks to further divide or son the subarrays sequentially, if the size of the subarrays is less than or equal to a predefined limit. Note that the program does not require to merge any of the subarrays. The parallel implementation of the quicksort algorithm has received considerable attention. The earliest one is the implementation on the $\mathrm{Cm}^{*}$ multiprocessor. It uses a stack to store the descriptors for a repeated subset of the array that has not yet been sorted. A descriptor is a pointer to the first element of the sub-array to be sorted. In fact, a descriptor is same as a task which holds the information about the 
indivisible computation. A similar implementation of the quickson algorithm is in [Evans \& Yousif 85]. In Evans and Yousif's work, a task queue is used to store the tasks generated at each level of the recursive call. The merge tasks of the parallel quicksort program are trivial and they are not present in the program. A parallel implementation of the Quick Sort algorithm is shown in Figure 3.5.

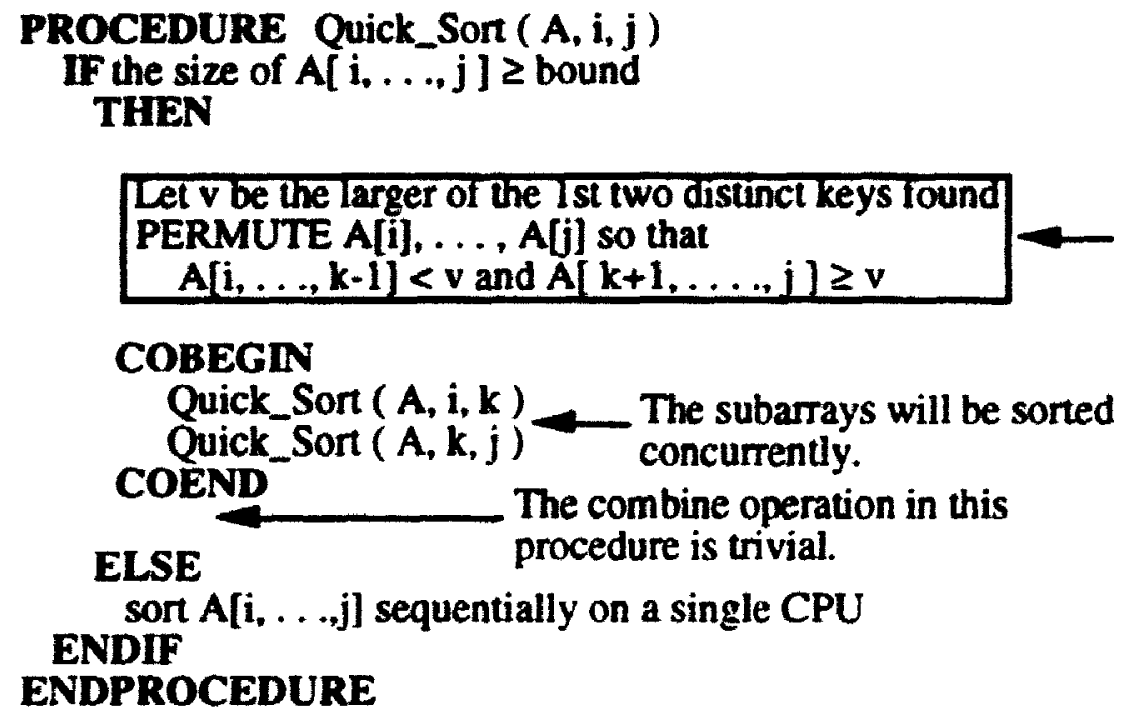

COBEGIN

Quick_Sort ( A, i, k) Quick_Sort ( A, k, j) The subarrays will be sorted COEND concurrently.

ELSE The combine operation in this procedure is trivial.

ENDIF

sort $A[i, \ldots, j]$ sequentially on a single $C P U$

\section{ENDPROCEDURE} into two subarrays. Tasks will be created to sort these subarrys.

Figure 3.5 A parallel implementation the Quicksort algorithm

\section{Example 2:Parallel Bubble-Merge Son}

Merge sort is a common sorting algorithm. In [Hagan et al. 85], a sorting algorithm called bubble-merge sort is implemented on the Denelcor HEP multiprocessur. Its parallel implementation is depicted in Figure 3.6. To accomplish the sorting, the original $n$ key population is partitioned into $d$ sets. For each of these sets, the bubble son program is used to sort these subarrays. Then, the parallel merge sort is used to merge the subarrays together until a single array is formed. 
PROCEDURE MERGESORT(low, high, p)

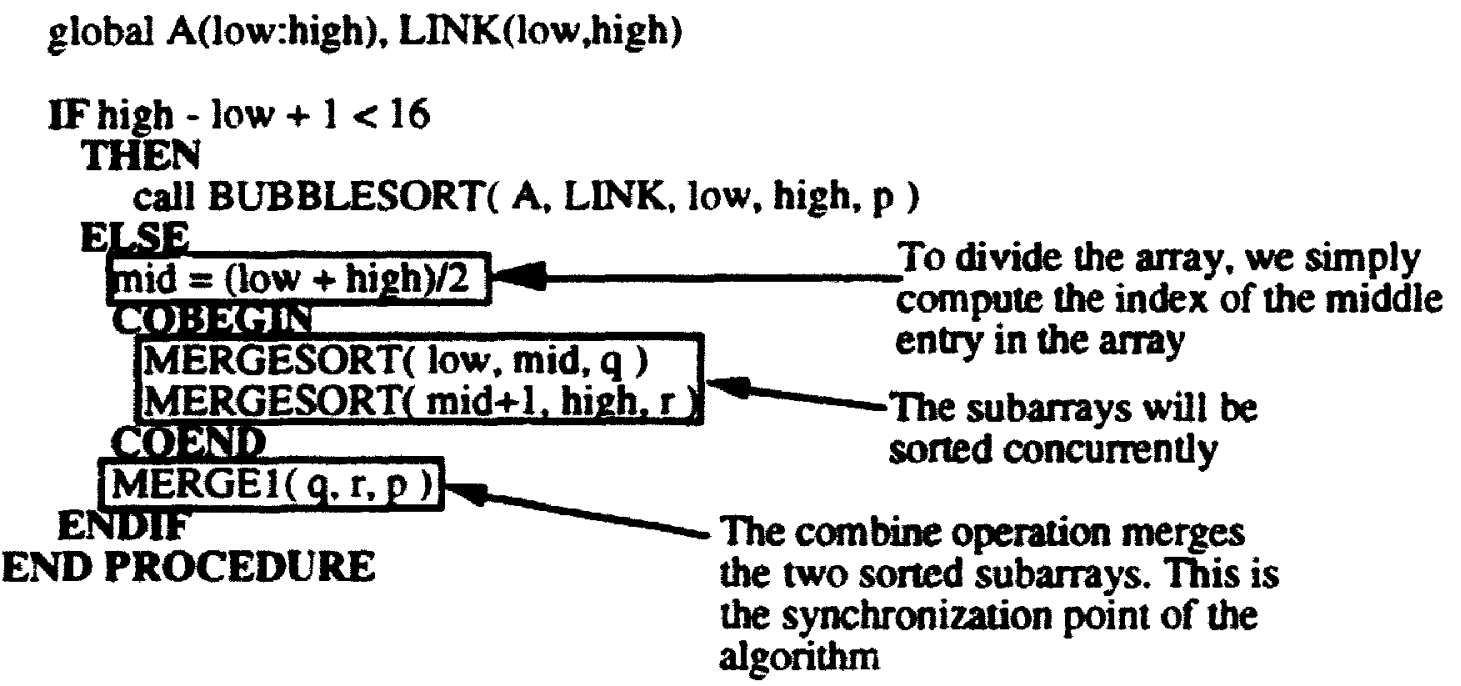

Figure 3.6 A parallel implementation of the mergeson algorithm

\section{Example 3: Parallel Adaplive Ouadrature}

In this section, we present a parallel implementation of an algorithm for solving the quadrature problem discussed in [Grit \& Mcgraw 85]. Given a continuous, nonnegative function $f(x)$, the quadrature problem is to compute the area bounded by $f(x)$, the $x$ axis, and vertical lines through $l$ and $r$. An illustration of the problem is shown in Figure 3.7. The algorithm begins with one interval from $l$ to $r$ and computes the midpoint $m$ between $l$ and $r$. It then calculates the areas of three trapezoids: (1) the large one bounded by $l, r, f(l)$ and $f(r)$ : (2) the small one bounded by $l, m, f(l)$, and $f(m)$; and (3) the small one bounded by $m, r, f(m)$, and $f(r)$. Next, the area of the larger trapezoid is compared with the sum of the areas of the two smaller ones. If these are sufficiently close, the area of the larger trapezoid is an acceptable approximation of the area under $f$. Otherwise, the process is repeated by solving the two subproblems of computing the area from $l$ to $m$ and from $m$ to $r$. The recursion continues until the approximation becomes acceptable. Figure 3.7 illustrates how the problem is subdivided. 


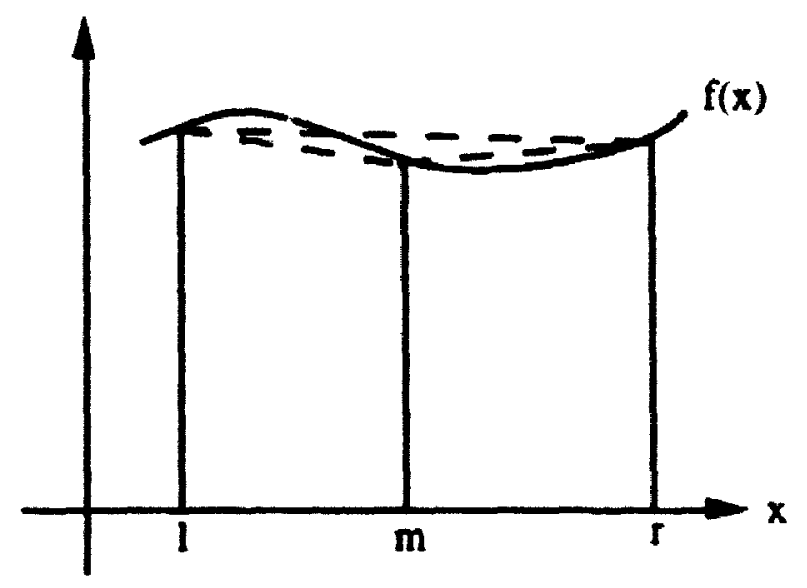

Figure 3.7 An iliustration quadrature problem

This is called adaptive quadrature since the solution adapts itself to the shape of the curve. In particular, in places where the curve is flat, a wide trapezoid will closely approximate the area. Hence new subproblems will not be generated. In places where the curve is changing shape, smaller and smaller subproblems will be generated as needed. An implementation of the parallel adaptive quadrature algorithm is given in Figure 3.8.

The procedure Adaptive_Quadrature has five input parameters. A left point $a$, a right point $b$, the values of $\mathrm{f}(\mathrm{a})$ and $\mathrm{f}(\mathrm{b})$, and the area of the trapezoid defined by these four points. (The area is included to avoid repeating the computation). It keeps on dividing the inputs until an acceptable answer is found. It is similar to the parallel Quicksort as they do not have an explicit combine operation. 
PROCEDURE Adaptive_Quadrature $(\mathrm{a}, \mathrm{b}, \mathrm{fa}, \mathrm{fb}$, area : real)

$\begin{aligned} & \text { tarea }:=(f(l)+f(r))^{*}(l+r) / 2 \\ & m:=(a+b) / 2 ; \\ & f m:=f(m)\end{aligned}$
$\begin{aligned} & \text { compute larea and rarea using trapezoids } \\ & \text { diff }:=\text { tarea - (larea }+ \text { rarea) }\end{aligned}$
$\begin{aligned} & \text { IF (diff }<=\text { epsilon) } \\ & \text { return tarea }\end{aligned}$
$\begin{aligned} & \text { ELSE } \\ & \text { computes the three } \\ & \text { different areas for the } \\ & \text { current iteration. And } \\ & \text { decides whether further } \\ & \text { division is needed }\end{aligned}$

\section{COBEGIN}

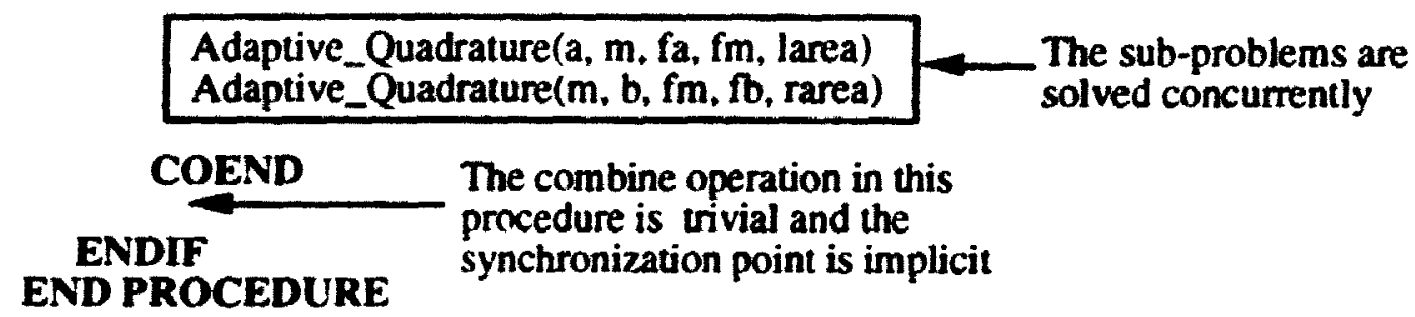

Figure 3.8 A parallel implementation of the adaptive quadrature algorithm

\section{Example 4: Parallel Convex Hull}

A parallel implementation of the convex hull algorithm is given in [Knecht 90]. The problem is stated as follows: given a point set $S$ consisting of $\boldsymbol{n}$ points in two dimensions, the convex hull is the boundary of the smallest convex domain in $\left(E^{2}\right)^{2}$ containing the point set $S$ [Knecht 90]. To compute the convex hull, the point set $\mathbf{S}$ is subdivided with respect to their $\mathrm{x}$ coordinates into two subproblems which can then be solved independently. The point set $\mathrm{S}$ is recursively divided into sub-sets until they contain at most three points. At each level of the recursive call, the convex hull of each subsets are combined together to form a convex hull. The parallel program is shown in Figure 3.9.

${ }^{2} E^{2}$ is the 2-1) Fuclidean space, that is the plane. Fach point in $E^{2}$ consists of two co-ordinates that is the $x$ and $y$ coordinates. 
PROCEDURE Parallel_CH (Input: a point set S)

Subdivide the point $S$ into $S_{1}=\left\{a_{1}, \ldots, \frac{a_{1}}{2^{n}}\right\}$ and

$S_{2}=\left(a_{1}{ }^{n+1}, \ldots, a_{n}\right)$

\section{COBEGIN}

Parallel_CH$\left(S_{1}\right)$, Parallel_CH$\left(S_{2}\right)$

COEND

Combine $\left(S_{1}, S_{2}\right)$

ENDPROCEDURE

Figure 3.9 A parallel implementation of the convex hull algorithm

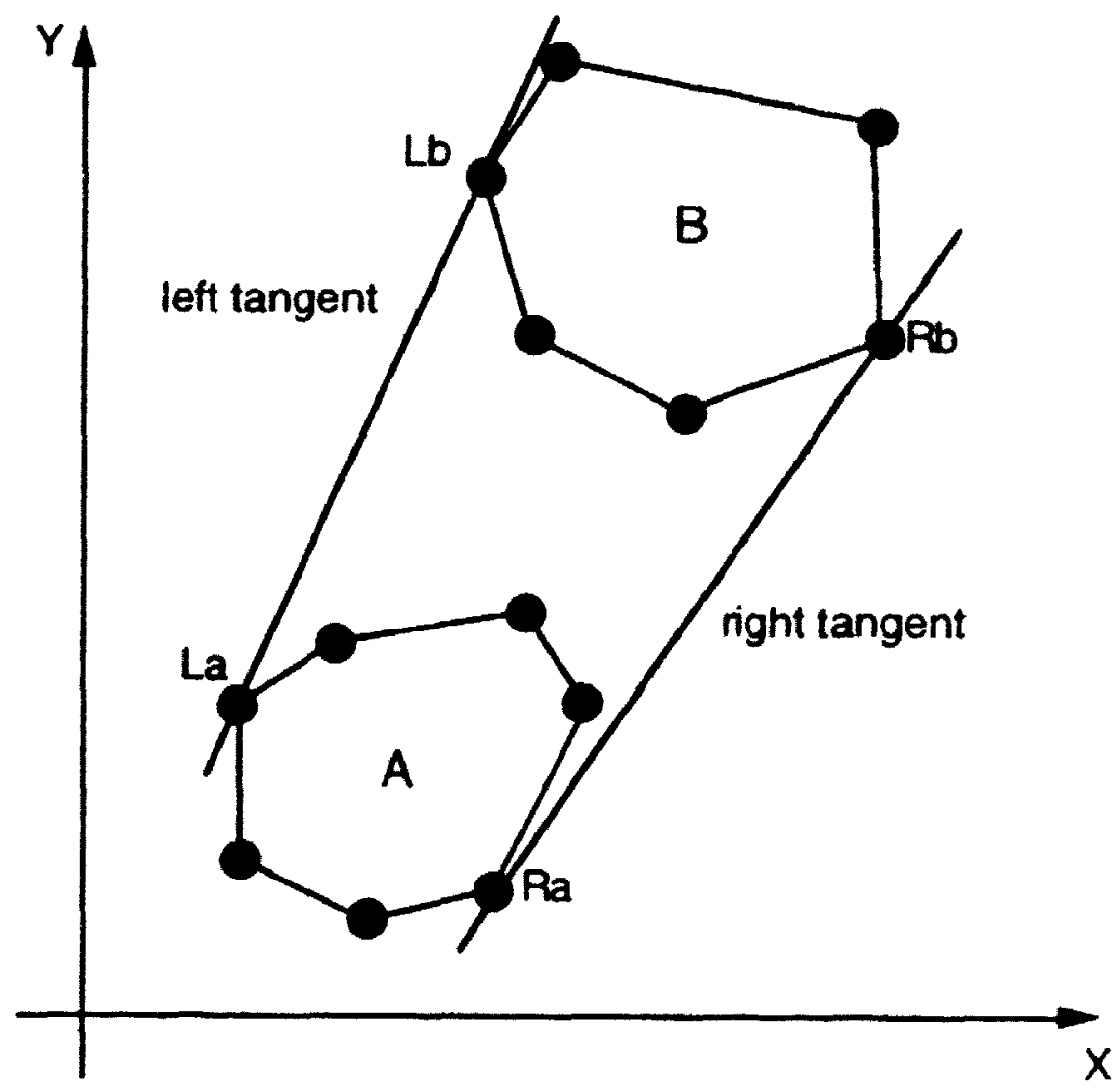

Figure 3.10 Illustration of the planar merge procedure

The combine operation proposed in [Preparata \& Hong 77] is used. It is assumed that the point set $S$ is sorted by their $x$-coordinates. Given two sub-convex hulls, the combine operation finds the ordered convex hull of a set of two-dimensional points, i.e. 
not only the set of vertices of the convex polygon representing the hull but also the sequence of the vertices on the boundary of this polygon. The left and right tangents are found by computing the $L_{b}$ and $R_{b}$ points of the convex polygon $B$, as well as the $L_{a}$ and $R_{a}$ points of the convex polygon $A . L_{b}$ is the leftmost point of the polygon $B$ and $R_{b}$ is the rightmost point of the polygon $\mathrm{B}$. Then, the resulting convex hull is computed by deleting the points that are bounded by the two tangents. Figure 3.10 uses an example to illustrate how the combine operation works.

\subsubsection{The Structure of Parallel Divide-and-Conquer}

The parallel implementation of divide-and-conquer algorithms exhibits a partitioning structure [Madala \& Sinciair 91]. The tasks created by this type of programs perform one of the following three operations: 1) splitting the inputs, 2) working on the inputs sequentially and 3) combining the outputs. Tasks that divide the input structures are called DIVIDE tasks. Tasks that work on the input structures sequentially are called WORK tasks. Tasks that merge the output are called MERGE tasks. The task system of parallel divide-and-conquer programs is depicted Figure 3.11 . It gives a high-level view of the structure of the parallel implementation of divide-and-conquer algorithms.

Corresponding to the program schome shown in Section 3.3.1, the DIVIDE rasks are those that performs the split-input operation. The WORK lask executes the code of the $A N S W E R$ procedure. The MERGE tasks execute the COMBINE operation. Generally, the execution time of the tasks decreases level by level over the upper half of the stucture, and increases level by level over the fower half of the structure. Synchronizations are done at the MERGE stage. 


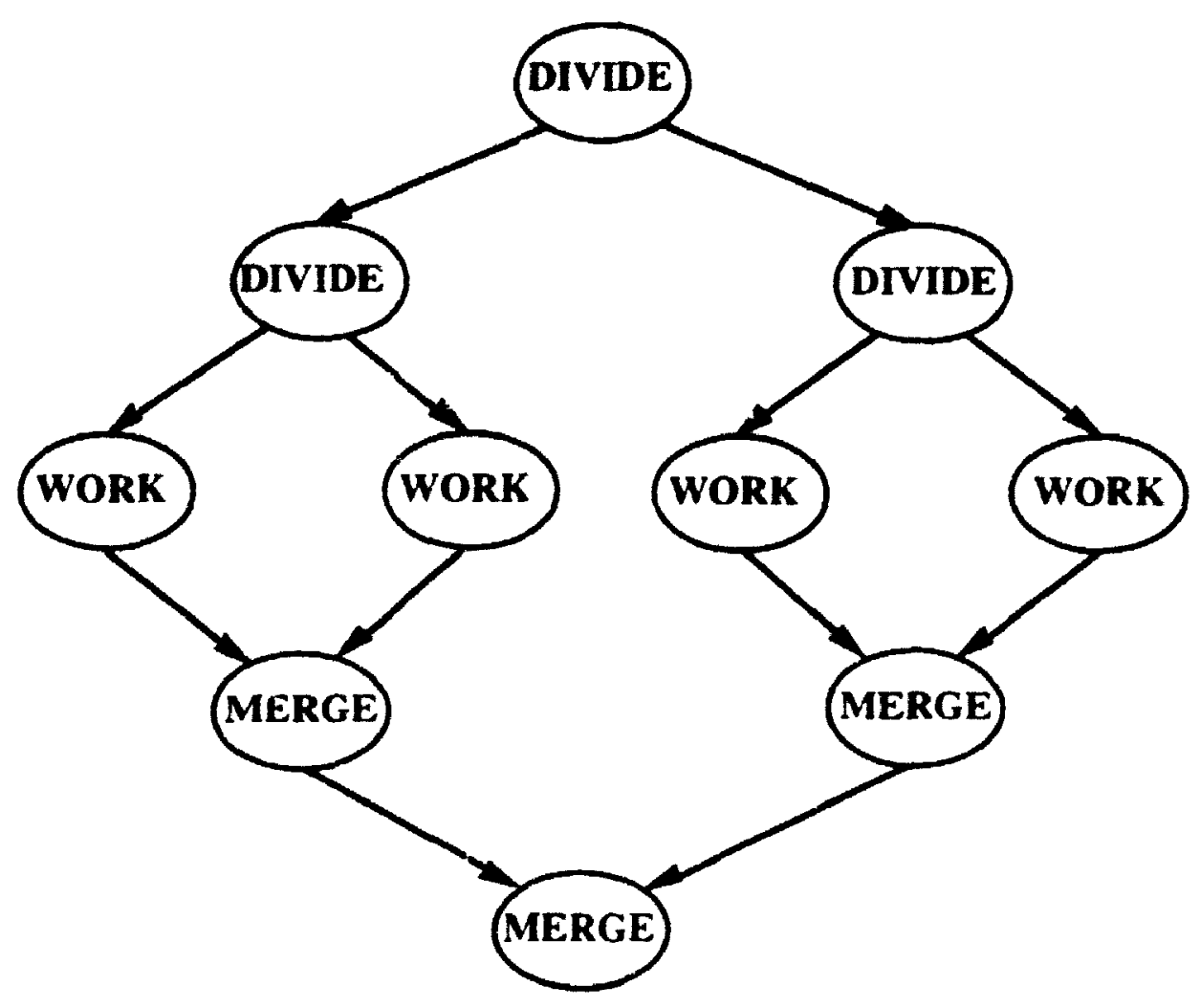

Figure 3.11 The task system of divide-and-conquer algorithm

In summary, we surveyed $\%$ number of parallel implementations of the divide-andconquer algorithms for the shared-memory multiprocessors. The examples given in this section only represent a subsel of algorithms that belong to this class.

\subsection{Parallel Matrix Factorization}

We are now going to discuss the implementation of a family of parallel algorithms for factorizing dense matrices. Factorization of dense linear matrices is an extremely important problem in the area of linear algebra. Once a matrix is factorized into some simpler form, its corresponding system of linear equations can be solved immediately. These algorithms use a method which computes the factorization directly from the matrix itself as opposed to finding an approximation to the factorization of the matrix. 


\subsubsection{Examples of Matrix Factorization Algorithm and Their Parallel Implementations}

\section{Example 1: The LU Decomposition Algorithm}

To solve a matrix equation of the form $A x=b$, one can decompose $A$ into upper $U$ and lower $L$ triangular matrices and then solve it by solving $L y=b$ and $U x=y$. The most computation intensive part of the algorithm is to find the LU decomposition. A LU decomposition algorithm, proposed by [Lord et al. 83], for the shared-memory multiprocessors will be reviewed. The process of forming the upper and lower triangular matrices relates to the direct method for solving the above matrix equation. Consider the direct methou of reducing a matrix by performing a series of elementary row operations. The only allowed operation is the addition of a multiple of one row to the rows below. We call this the basic row operation.

$$
A_{1}=\left[\begin{array}{ccc}
2 & 4 & -2 \\
1 & -1 & 5 \\
4 & 1 & -2
\end{array}\right]
$$

If $\frac{1}{2}$ times the first row is subtracted from the second and 2 times the first subtracted from the third row, the result will be the matrix

$$
A_{2}=\left[\begin{array}{ccc}
2 & 4 & -2 \\
0 & -3 & 6 \\
0 & -7 & 2
\end{array}\right]
$$

in which the first column of the second and third rows is reduced to zeros. The second column of the third row can be reduced to zero by subtracting $\frac{7}{3}$ times the second row. The result is as follow's.

$$
A_{3}=\left[\begin{array}{ccc}
2 & 4 & -2 \\
0 & -3 & 6 \\
0 & 0 & -12
\end{array}\right]
$$


From the above row reduction, we obtain an upper triangular matrix $A_{3}$. Each of the above reductions corresponds to a premultiplication of an elementary triangular matrix ${ }^{3}$, where

$$
M_{1}=\left[\begin{array}{rrr}
1 & 0 & 0 \\
-\frac{1}{2} & -1 & 0 \\
-2 & 0 & -1
\end{array}\right] \quad M_{2}=\left[\begin{array}{ccc}
1 & 0 & 0 \\
0 & -1 & 0 \\
0 & -\frac{7}{3} & -1
\end{array}\right]
$$

We can see that $A_{3}=M_{2} M_{1} A_{1}$. As $M_{1}$ and $M_{2}$ are non-singular matrices and their inverse $M_{1}^{-1}, M_{2}^{-1}$ exist. Thus, from the above equation, we have

$$
A_{1}=M_{1}^{-1} M_{2}^{-1} A_{3}
$$

Therefore, a matrix can be reduced to an upper triangular matrix $A_{3}$ by premultiplying a series of elementary matrices. Since $\mathbf{M}_{1}$ anc $\mathbf{M}_{2}$ are lower triangular matrices, the product of $M_{1}$ and $M_{2}$ must be a lower triangular matix. If we let, $L=M_{1}^{-1}$ $M_{2}^{1}=M_{1} M_{2}$ and $U=A_{3}$, we have $A_{1}=L U$, which is the $L U$ decomposition of the matrix $A_{1}$.

In general, given a matrix $A=M_{1}^{-1} M_{2}^{-1} \ldots M_{k}^{-1} \ldots M_{n}^{-1} A_{n}$ where the $M_{i}$ 's are elementary lower triangular matrix and $A_{n}$ is an upper triangular matrix, then $L=M_{1}^{-1} M_{2}^{-1}$. $\ldots M_{k}^{-1} \ldots M_{v}^{-1}$ and $U=A_{n}$ is the $L U$ decomposition of $A$.

The LU decomposition algorithm is shown in Figure 3.12. The main body of the algorithm is the $\mathrm{k}$ loop. In its $k^{\text {th }}$ iteration, the $k^{\text {th }}$ pivor column is first computed. The pivot column consists of the multipliers for performing the basic row operations ${ }^{4}$ in the $k^{\text {th }}$ reduction of the matrix $A$. And, it is equal to $\left[a_{k k}, \frac{a_{(k+1) k}}{a_{k k}} \ldots, \frac{a_{n k}}{a_{k k}}\right]$. The multipliers are

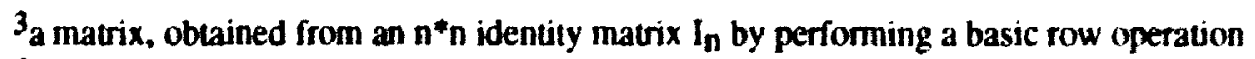
${ }^{4}$ for the definition of basic row operation, see page 32 . 
then used to perform the basic row operations on the submatrix to its right. An illustration of the reduction is shown in Figure 3.13.

PROCEDURELU_Decomp ( A(n,n) )

FOR $k:=1$ TO $n-1$ DO
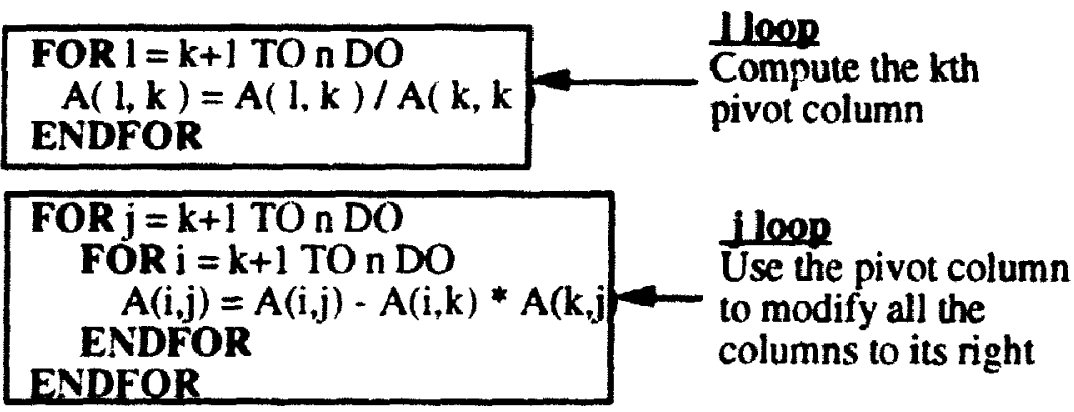

ENDFOR

\section{ENDPROCEDURE}

Figure 3.12 The LU Decomposition algorithm

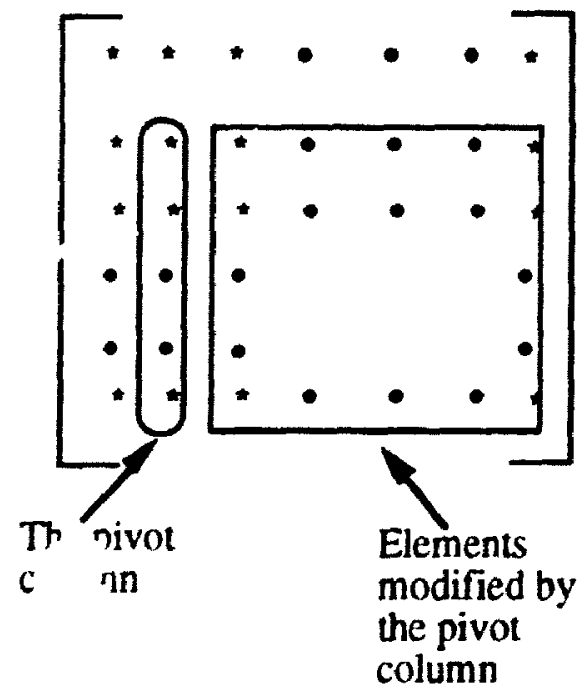

Figure 3.13 An illustration of the reduction where $k=2$

This algorithm works fine as long as there is no small pivot ${ }^{5}$ during the reduction of the matrix A. Division of a small pivot results in a large rounding error that propagates through the execution. The problem will be very severe if the algorithm is implemented on

\footnotetext{
5 A pivot: the first clement in the pivot colurnn
} 
computers that perform finite digit arithmetic. Consider the following example that illustrate the problem of having a small pivot. A small pivot is equal to 0.001 , located in the upperleftmost comer of the matrix $A_{1}$.

$$
A_{1}=\left[\begin{array}{ccc}
0.001 & 2.000 & 3.000 \\
-1.000 & 3.712 & 4.632 \\
-2.000 & 1.072 & 5.643
\end{array}\right]
$$

Then a four-digit realization of the reduction of $A$ to upper triangular form produces the following matrices.

$$
\begin{aligned}
& \mathrm{M}_{1}=\left[\begin{array}{ccc}
1.000 & 0.000 & 0.000 \\
1000.0 & 1.000 & 0.000 \\
2000.0 & 0.000 & 1.000
\end{array}\right] \\
& \mathrm{A}_{2}=\left[\begin{array}{ccc}
0.001 & 2.000 & 3.000 \\
0.000 & 2004.0 & 3005.0 \\
0.000 & 4001.0 & 6006.0
\end{array}\right] \\
& \mathrm{M}_{2}=\left[\begin{array}{ccc}
1.000 & 0.000 & 0.000 \\
0.000 & 1.000 & 0.000 \\
0.000 & -1.997 & 1.000
\end{array}\right] \\
& \mathrm{A}_{3}=\left[\begin{array}{ccc}
0.001 & 2.000 & 3.000 \\
0.000 & 2004.0 & 2005.0 \\
0.000 & 0.000 & 5.000
\end{array}\right]
\end{aligned}
$$

The (3,3)-element of the resultung matrix $A_{3}$ has very large round off error, that is the resulting value is 5.000 compared to the real value of 5.922 . However, if the first and second row of the matrix are interchanged, the matrix can be reduced with yery little numerical error. This new matrix is stated as follows. It is equivalent to $A_{1}$ and can be reduced without any difficulty.

$$
A_{3}=\left[\begin{array}{ccc}
-1.000 & 3.712 & 4.623 \\
0.001 & 2.000 & 3.000 \\
-2.000 & 1.072 & 5.643
\end{array}\right]
$$


From this observation, we see that the algorithm should be modified in such a way that it must search the pivot column for a maximum element and perform row interchange to make the maximum element to become the pivot. The algorithm for computing $L U$ decomposition with partial pivoting is given in Figure 3.14.

PROCEDURE LU_Decomp $(A(n, n))$

FOR $k:=1-O n-1$ DO

Find 1 such that $\quad$ Finds the maximum $|A(1, k)|=\max (|A(k, k)|, \ldots,|A(n, k)|) \longrightarrow$ element in the pivot PIV(k) $<-1$

EXCHANGE ( $A(P I V(k), k), A(k, k)$ row interchange

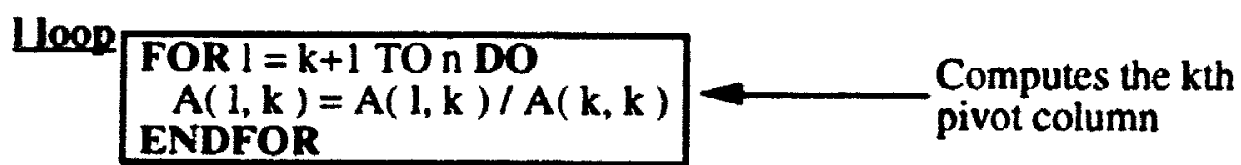

$1000 \begin{aligned} & \text { FOR } \mathrm{j}=\mathrm{k}+1 \text { TO } \mathrm{\text {DOO}} \\ & \text { EXCHANGE( A(PIV }(k), \mathrm{j}), \mathrm{A}(\mathrm{k}, \mathrm{j}) \\ & \text { FOR } \mathrm{i}=\mathrm{k}+1 \text { TO } \mathrm{\text {DO }} \\ & \mathrm{A}(\mathrm{i}, \mathrm{j})=A(\mathrm{i}, \mathrm{j})-\mathrm{A}(\mathrm{i}, \mathrm{k}) * A(k, j) \\ & \text { ENDFOR } \\ & \text { ENDFOR }\end{aligned}$

Perform row interchange and the basic row operations

\section{ENDFOR}

\section{ENDPROCEDURE}

Figure 3.14 The algorithm for LU decomposition with partial pivoting

A number of researchers have worked on parallelizing the above algorithm. The most common approach is to derive a task system for the above algorithm and perform static scheduling on this task system [Lord et al. 83].

An implementation of the LU decomposition on the DASH shared-memory multiprocessor is discussed in [Gupta et al. 91]. In this implementation, the matrix is decomposed on a columnwise basis. Columns are statically assigned to the processors in an interleaved fashion. Starting from left to right, columns are used to modify all columns to their right. Processors wait until the columns owned by them are ready to be modified. 
Then they use the pivot column to modify all columns owned by them to the right of the pivot. When a processor finishes a column, it releases all the processors that are waiting for that column.

Another parallel implementation is to have processes for each column of the matrix. At each iteration, tasks that perform the operation on the columns are created and assigned to the processes. We assume that only one task is assigned to a process, in each iteration. There are two shared variables in this program, namely, the matrix $A$ and an array of Pivot record. A pivot record contains two fields, flag and value. The flags in the pivot record are used to ensure that a pivot is computed before it is being used. We consider this implementation of the algorithm and it is shown in Figure 3.15.

The program begins its execution by creating processes for each column of the matrix. During the execution, tasks are created and assigned to the processes. Whe , a processor becomes idle, a process (or a task) is scheduled to run on it. We assume that each processor has local memory. When it starts the computation for task $i$, it copies all the shared variables that will be accessed prior to performing the computation for task $i$. The concurrence control mechanism for this algorithm is locking. A task locks the column of the matrix before it performs any modification on it. The column will be released when the computation on that column is completed.

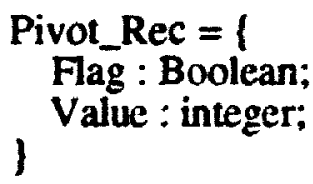

Shared Variables [A(1:n, $1: n)$ of real, Pivot(1:n) of Pivot_Rec]

Global initialize ( A, Pivot)

TASK Multiply_Column local Variables( col( $1: n)$, locPiv:Pivot_Rec]

FOR $k=1$ TO $n-1$ DO

IF $\left(k==t a s k \_I d\right)$

$\operatorname{lock}(A(1: n), k))$ $\operatorname{get}(A(1: n, k), \operatorname{col}(1: n))$ 


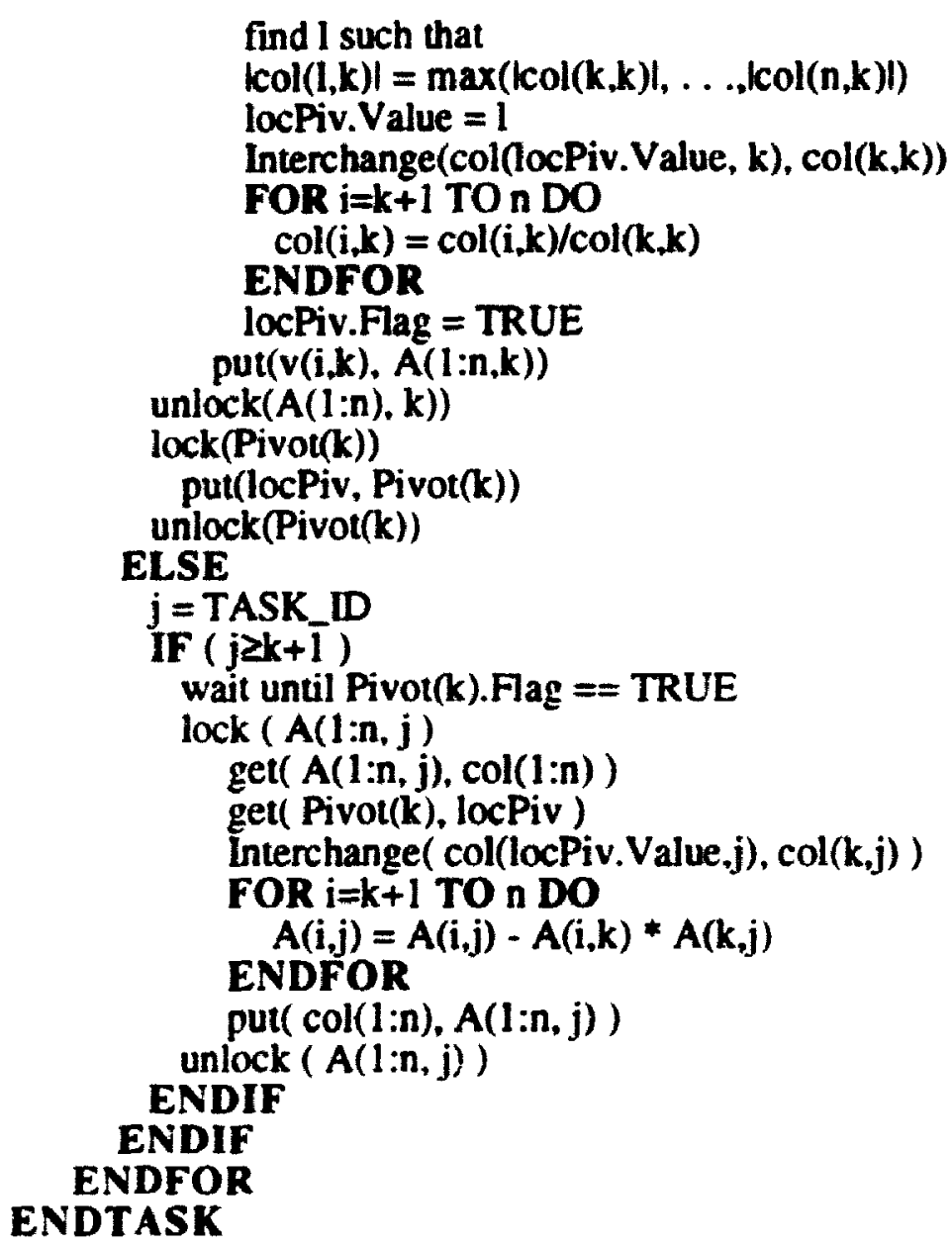

Figure 3.15 A parallel implementation of the $\mathrm{LU}$ decomposition algorithm

\section{Example 2: The Gauss-Jordan Algorithm}

The Gauss-Jordan method is used to solve a linear system of equations. It is similar to the LU decomposition algorithm except that in the $k^{\text {th }}$ pivot column modification, not only the row $i+1$ to $n$ are modified but also the 1 to $i-1$ rows. At the end, it gives a diagonal matrix which is the result of solving a system of linear equations. The algorithm for computing the Gauss-Jordan method is shown in Figure 3.16. 


\section{PROCEDURE Gauss_Jordan ( $\mathbf{A}(\mathbf{n}, \mathbf{n}))$}

FOR $\mathrm{k}:=1$ TO $\mathrm{n}-1 \mathrm{DO}$

\begin{tabular}{|c|c|c|}
\hline & $\begin{array}{l}\text { Find l such that } \\
|A(\mid, k)|=\max (|A(k, k)|, \ldots,|A(n, k)|) \\
\text { PIV(k)<-1 } \\
\text { EXCHANGE ( A(PIV(k), k ), A(k,k) }\end{array}$ & $\begin{array}{l}\text { Find maximum } \\
\text { element of the pivot } \\
\text { column and perform } \\
\text { row interchange }\end{array}$ \\
\hline Lloope & $\begin{array}{l}\text { FOR } 1=k+1 \text { TO n DO } \\
A(1, k)=A(1, k) / A(k, k) \\
\text { ENDFOR }\end{array}$ & $\begin{array}{l}\text { Compute the pivot } \\
\text { column }\end{array}$ \\
\hline Jloon & $\begin{array}{l}\text { FOR } j=k+1 \text { TO n DO } \\
\text { EXCHANGE (A(PIV }(k), k) . A(k, j) \\
A(k, j)=A(k, j) / A(k, k) \\
\text { FOR } i=1 \text { TO } n \text { DO } \\
\text { IF }(i \neq k) \\
A(i, j)=A(i, j)-A(i, k) * A(k, j) \\
\text { ENDFOR }\end{array}$ & $\begin{array}{l}\text { Add multiple of the } \\
\mathrm{kth} \text { row to the } 1, \ldots, \\
\mathrm{k}-1, \mathrm{k}+1, \ldots, \mathrm{n} \text { th } \\
\text { rows. }\end{array}$ \\
\hline
\end{tabular}

ENDFOR

\section{ENDPROCEDURE}

Figure 3.16 The Guass-Jordan algorithm

Since the Gauss-Jordan algorithm is more or less the same as the LU algorithm, its parallel version is very similar to the previous one. A parallel implementution of this algorithm is shown in Figure 3.17. The synchronization methods as well as the method for ensuring mutually exclusive access of a shared variable are the same as those LU decomposition.

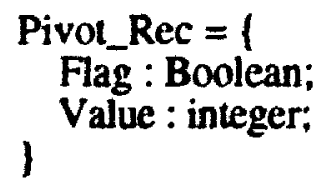

Shared Variables [A(1:n, 1:n) of real, Pivot(1:n) of Pivot_Rec] Global initialize ( $A$, Pivot)

TASK Multiply_Column local Variables[ col(1:n), locPiv:Pivot_Rec]

FOR $k=1$ TO $n-1$ DO

IF ( $k==$ task_Id) $\operatorname{lock}(A(1: n), k))$ 


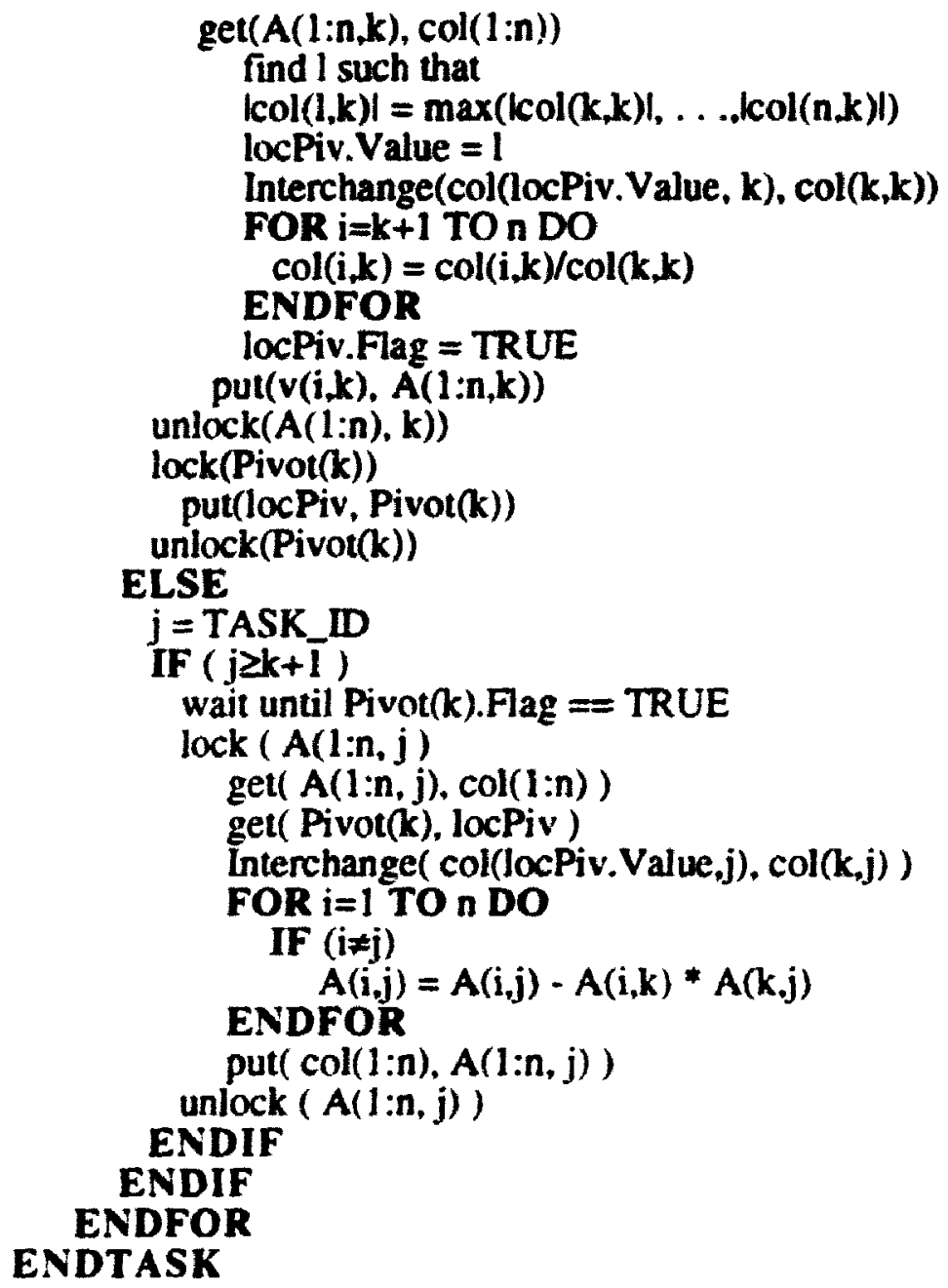

Figure 3.17 A parallel implementation of the Gauss-Jordan algorithm

\section{Example 3: The Cholesky Decomposition Algorithm}

A symmetric matrix $A$ is positive definite, if $x \neq 0 \Rightarrow x A x>0$. Let $A$ be a positive definite matrix. The Cholesky decomposition of $A$ is $L$ and $L T$, such that $A=L L T$. Consider the following positive definite matrix, $\left[\begin{array}{cc}6 & 11 \\ 11 & 34\end{array}\right]$.

Its Cholesky decomposition is given by 


$$
L=\left[\begin{array}{cc}
\sqrt{6} & 0 \\
\frac{11}{\sqrt{6}} & \sqrt{\frac{83}{6}}
\end{array}\right] \text { and } L^{T}=\left[\begin{array}{cc}
\sqrt{6} & \frac{11}{\sqrt{6}} \\
0 & \sqrt{\frac{83}{6}}
\end{array}\right] \text {. }
$$

The Cholesky factorization algorithm is derived based on the matrix equation shown below

$$
A=\left[\begin{array}{ll}
a_{11} & \sigma^{T} \\
\sigma & B
\end{array}\right]=\left[\begin{array}{cc}
\sqrt{a_{11}} & 0 \\
\frac{\sigma}{\sqrt{a_{11}}} & I_{n-1}
\end{array}\right]\left[\begin{array}{cc}
1 & 0 \\
0 & A_{1}
\end{array}\right]\left[\begin{array}{cc}
\sqrt{a_{11}} & \frac{\sigma^{T}}{\sqrt{a_{11}}} \\
0 & I_{n-1}
\end{array}\right]
$$

$\Phi$ is a column vector which is the first column of $A$ without $a_{11}$ and by equating both sides of the equation we get $A_{1}=B \frac{\omega \sigma^{T}}{a_{11}}$.

From the above equation, we see that if the Cholesky factorization of $A_{1}$ is $L_{1} L_{1}^{T}$. where $L$ is a lower triangular matrix then we can substitute $A_{i}$ by $L_{1} L_{1}^{T}$. Hence, we have

$$
A=\left[\begin{array}{cc}
\sqrt{a_{11}} & 0 \\
\frac{\sigma}{\sqrt{a_{11}}} & I_{n-1}
\end{array}\right]\left[\begin{array}{cc}
1 & 0 \\
0 & A_{1}=L_{1} L_{1}^{T}
\end{array}\right]\left[\begin{array}{cc}
\sqrt{a_{11}} & \frac{\sigma^{T}}{\sqrt{a_{11}}} \\
0 & I_{n-1}
\end{array}\right]
$$

and by some algebraic manipulations on the matrices, we have

$$
A=\left[\begin{array}{cc}
\sqrt{a_{11}} & 0 \\
\frac{\sigma}{\sqrt{a_{11}}} & L_{1}
\end{array}\right]\left[\begin{array}{cc}
\sqrt{a_{11}} & \frac{\sigma^{T}}{\sqrt{a_{11}}} \\
0 & L_{1}^{T}
\end{array}\right]
$$

Therefore, the Cholesky factorization of $\mathrm{A}$ is $\mathrm{LL}^{\mathrm{T}}$ where

$$
L=\left[\begin{array}{ll}
\sqrt{a_{11}} & 0 \\
\frac{\sigma}{\sqrt{a_{11}}} & L_{1}
\end{array}\right]
$$

Equation 3.1 
Thus, the Cholesky factorization of A can be found by repeatedly performing the reduction over the submatrices with decreasing size given in the equation 3.1. The Cholesky factorization algorithm is shown in Figure 3.18. The algorithm is very similar to the LU decomposition, that is, they both make use of a pivot column to perform the basic row operations on the submatrix to its right. The only difference is the way the pivot column is defined. In this algorithm, the $k^{\text {th }}$ pivot column is equal to $\left[\sqrt{a_{k k}} \cdot \frac{a(k+1) k}{\sqrt{a_{k k}}}, \ldots\right.$ $\left.\frac{a_{\text {nk }}}{\sqrt{a_{\text {kk }}}}\right] \mathrm{T}$. The parallel implementation of this algorithm is shown in Figure 3.19.

PROCEDURE Cholesky_Decomp( $A(n, n))$
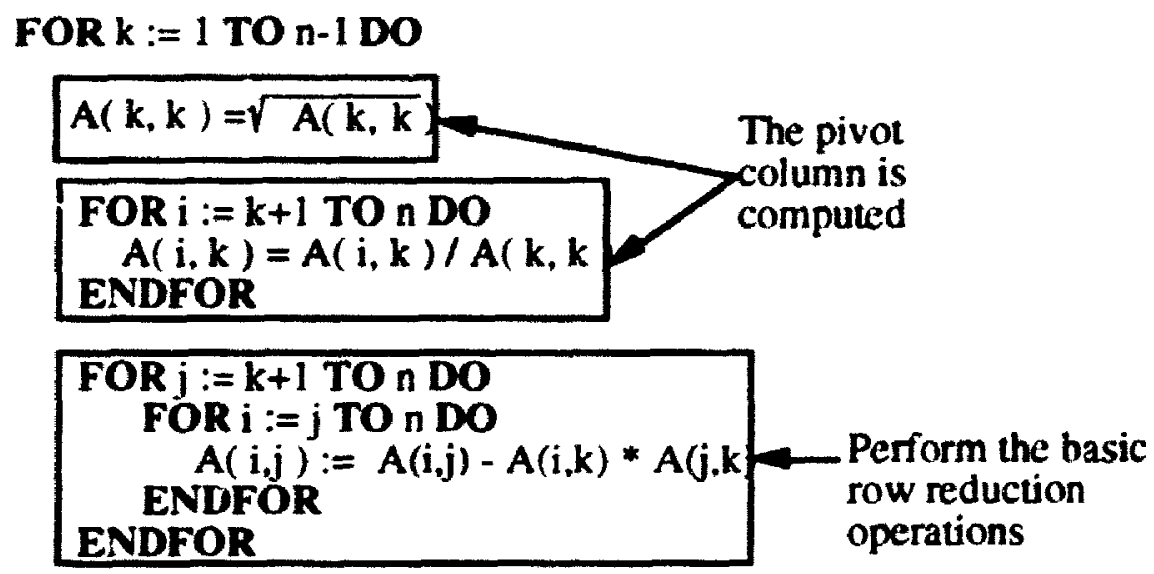

\section{ENDFOR}

\section{ENDPROCEDURE}

Figure 3.18 The Cholesky factorization algorithm

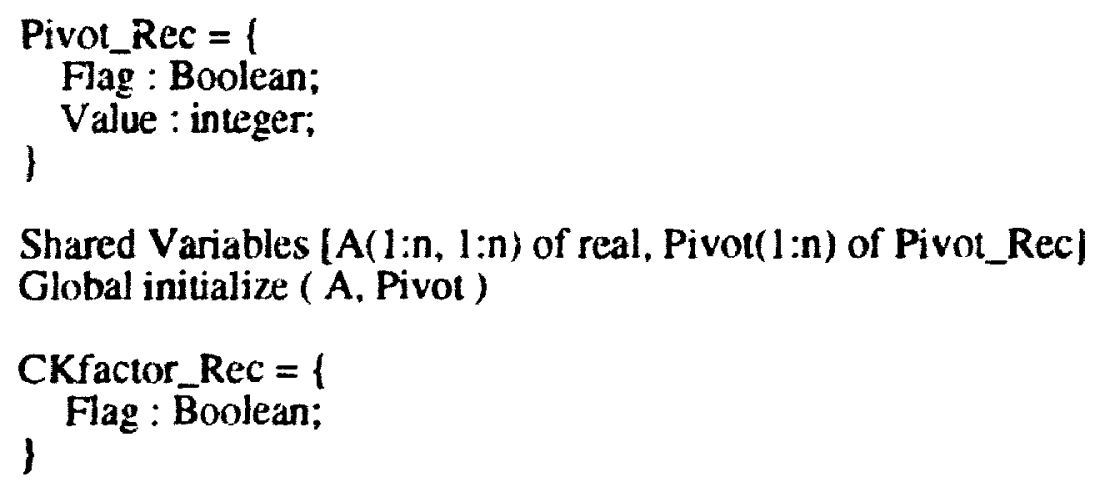




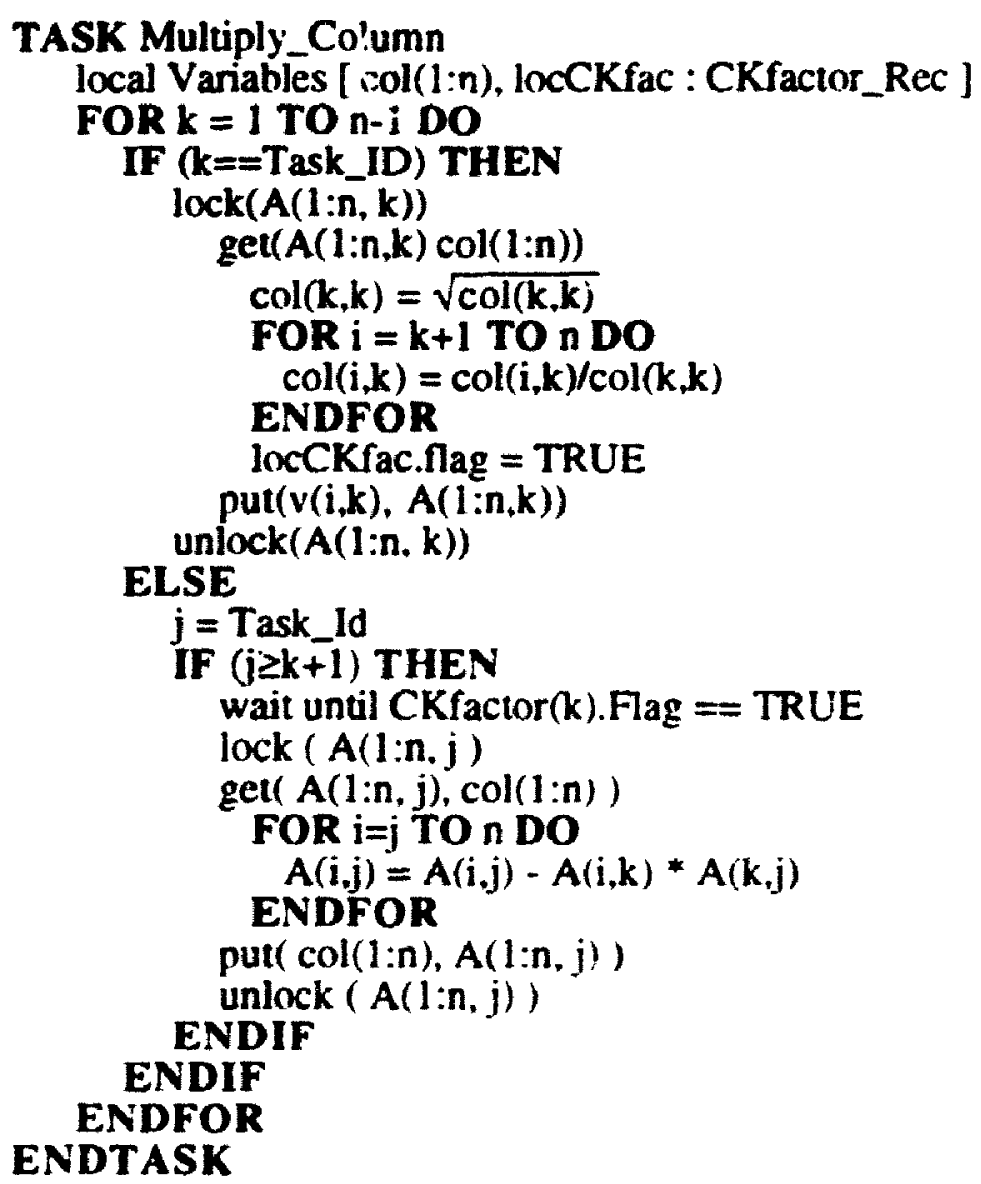

Figure 3.19 A parallel implementation of the Cholesky factorization algorithm

\subsubsection{The Structure of Parallel Matrix Factorization}

The siructure of these parallel programs is represented by a task system shown in Figure 3.20. $\mathrm{T}_{i}^{j}$ represents the task that works on column $i$ in the $j^{\text {th }}$ iteration of the outermost loop. 


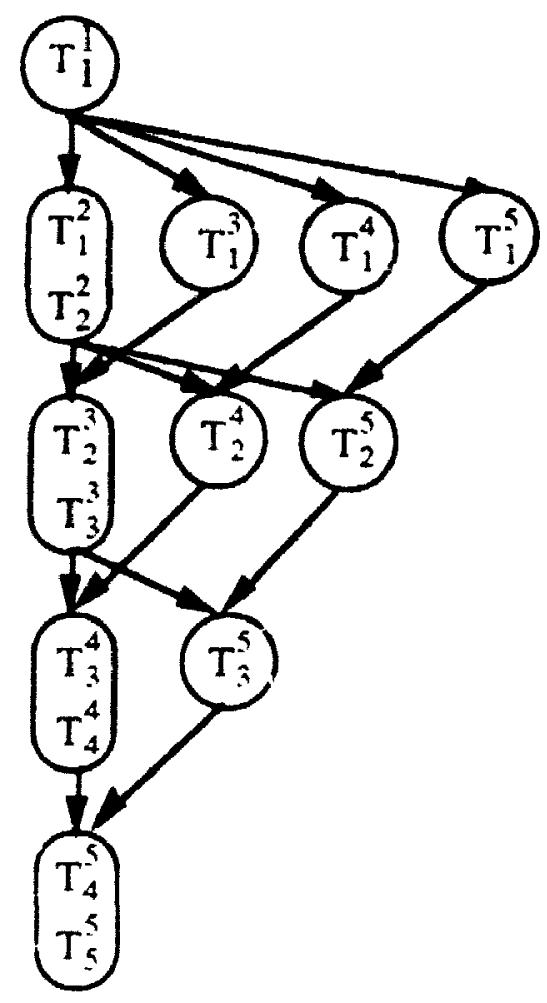

Figure 3.20 The task graph of matrix factorization

However, the actual function of the tasks are different for each of the above mentioned algorithms. In the LU decomposition and Gauss Jardon algorithms, $T_{k}^{k}$ finds the pivot element. For the Cholesky factorization algorithm, $T_{k}^{k}$ computes the square root of the diagonal element and then divides the lower par of column $k$ by that square root. In all the three algorithms, $T_{k}^{j}$ uses the elements of column $k$ to modify i'use in column $j$.

As mentioned in /Lord et al. 83], $T_{k}^{k}$ should have additional importance because they have a large number of outgoing edges. Much research has been done to find an optimal static scheduling for this type of parallei computation. However, our major concern is the performance of scheduling polecies for general-purpose parallel systems. Since matrix factorization is a way of performing Gaussian elimination on a matrix, from now on we call this class of programs as Gaussian elimination. 


\subsection{Parallel Search}

Searching is a common method for solving problems in artificial intelligence. In general, a search system consists of three main components. The first of these is a database, which describes both the current state and the goal. Consider the problem of proving a theorem. In this case, the current state consists of the assertions representing the axioms, lemma, and semantic networks. Th.e goal is an assertion representing the theorem to be proved. The second component of the search system is a set of operators that are used to manipulate the database. In the case of a theorem-proving system, operations are the rules of inference such as modus ponens or resolution. The third component is the control strategy for deciding the next action, what operator to apply and where to apply it. The choice of a control strategy affects the contents and organization of the database. The process of problem solving is to bring the problem state forward from its initial configuration to one satisfying a goal condition by repeatedly applying an operator.

\subsubsection{Breadth-first on State-Space Graphs}

The search system mentioned above advances the search by applying an operator to produ. a new state in the database. A state-space representation of a problem employs two kinds of entities: stotes, which are data structures that give "snapshots" of the problem at each stage of the problem solving, and operators applied to transiom the problem from one state to another. We use the 8-puzzle game to illustrate the basic concept of the statespace representation. An 8-puzzle is a square tray containing eight square tiles of equal size, numbered 1 to 8 . The space for the ninth tile is vacant. 


\begin{tabular}{|l|l|l|}
\hline 2 & 1 & 6 \\
\hline 4 & & 8 \\
\hline 7 & 5 & 3 \\
\hline
\end{tabular}

Figure 3.21 An example of the 8-puzzle game

The tiles can be moved by sliding them vertically or horizontaily into the em;n. square. The problem is to transform some initial tile configuration to a special final configuration. The state-space representation of the problem is giver, as follows. A state is a particular configuration or arrangement of tiles; each state might be represented by a $3 \times 3$ matrix. The operators are the possible moves that are made by the empty square. Thus, four operators are to be used.

UP
DOWN $\quad$ Move the blank up one square,
LEFT
RIGHT Move the blank left one square.

The four operators defined for the 8-puzzle form a set of partial functions on the state space. A state space problem can be interpreted as a triple $(S, O, G)$, where $O$ represents the set of operators, $S$ represents the set of initial states and $G$ the set of goal states. A solution to the problem is a finite sequence of applications of operators that changes an initial state into a goal state.

A state space can be treated as a directed graph whose nodes are states and whose ares are operators transforming from one state to another. Consider the 8-puzzle example. Its state-space graph representation has the following property. For each node, if it does 
not represent a goal state, it will in turn create four nodes corresponding to the four operators as mentioned above. In Figure 3.22, node 1 represents the initial state of the game, and four different nodes will be created depending on the four different operators. Nodes 2, 3, 4 and 5 are called the successors of node 1 . Note that the node 2, 3, 4 and 5 will in turn generate their successors and this process continues until the goal state is reached.

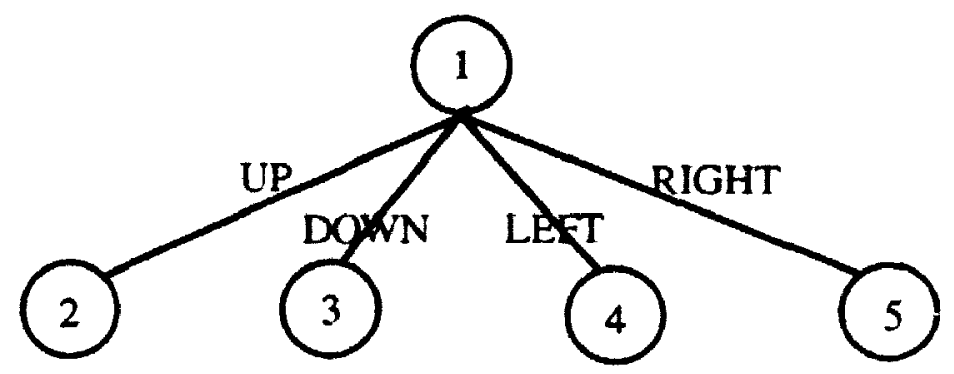

Figure 3.22 A state-space graph

The search for a solution is conducted by making explicit just enough of the statespace graph to contain a solution path. If the order in which potential solution paths are considered is arbitrary, using no domain-specific information to judge where the solution is likely to lie, the search is called blind search. Tnere are mainly two blind search methods: the breadth-first and the depth-first search. They differ from one another mainly in the order in which nodes are examined. In each case, it is assumed that there is a procedure for finding all the successors of a given node; that is, all the states that can be reached from the current state by a single operator application. Such a procedure is said to expand the given node. However, we will only consider the breadth-first search of a state-space graph. The algorithm makes the following two assumptions.

1) The state-space graph is a tree. The implication is that there is only one star state (the root) and that the path from the star node to any other node is unique. 
2) Whenever a node is expanded, creating a node for each of its successors, the successor nodes contain pointers back to the parent node. When a goal node is finally generated. this feature makes it possible to trace the solution path.

The breadth-first search method expands nodes in order of their proximity to the start node, measured by the depth of the node. The depth of a node is the number of arcs between the start node and the given node. In other words, it considers every possible operator sequence of lengtil $n$, before any sequence of length $n+l$. Thus, although the search may be an extremely long one, it is guaranteed eventually to find the right solution sequence if any solution exists.

The breadth-first search is described by the following algorithm:

1. Place the root node on a list, called OPEN, of unexpended nodes. If the start node is a goal node, a solution has been found.

2. If OPEN is empty, no solution exists.

3. Remove the first node, $n$, from OPEN and place it in a list, called CLOSED, of expanded nodes.

4. Expand node $n$. If it has no successors, go to (2).

5. Place all the successors of node $n$ at the end of the OPEN list.

6. If any of the successors of node $\mathrm{n}$ is a goal node, a solution has been found. Otherwise, it goes to (2).

We consider a simple protlem that involves changing the order of three blocks. The injtial state of the world is that blocks 2 and 3 are on the table, and block 1 is on top of block 2 . We want to reach a goal state in which the three blocks are stacked with block 1 on 
top, block 2 in the middle, and block 3 on the bottom. The problem is depicted in Figure 3.23 .

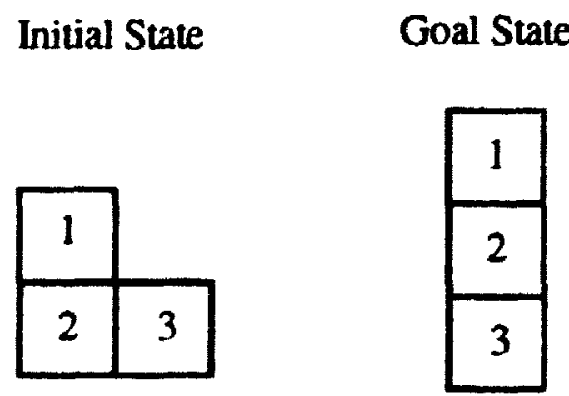

Figure 3.23 The configuration of the problem of changing three blocks

The only operator is MOVE X to $\mathrm{Y}$, which moves object $\mathrm{X}$ on top of another object, $Y$. As preconditions to applying the operator, it is required (a) that $X$, the object to be moved, be a block with nothing on top of it and (b) that if $\mathrm{Y}$ is a block, there must be nothing on Y. Finally, the operator is not to be used to generate the same state more than once. Figure 3.24 shows the search tree generated by the breadth-first algorithm.

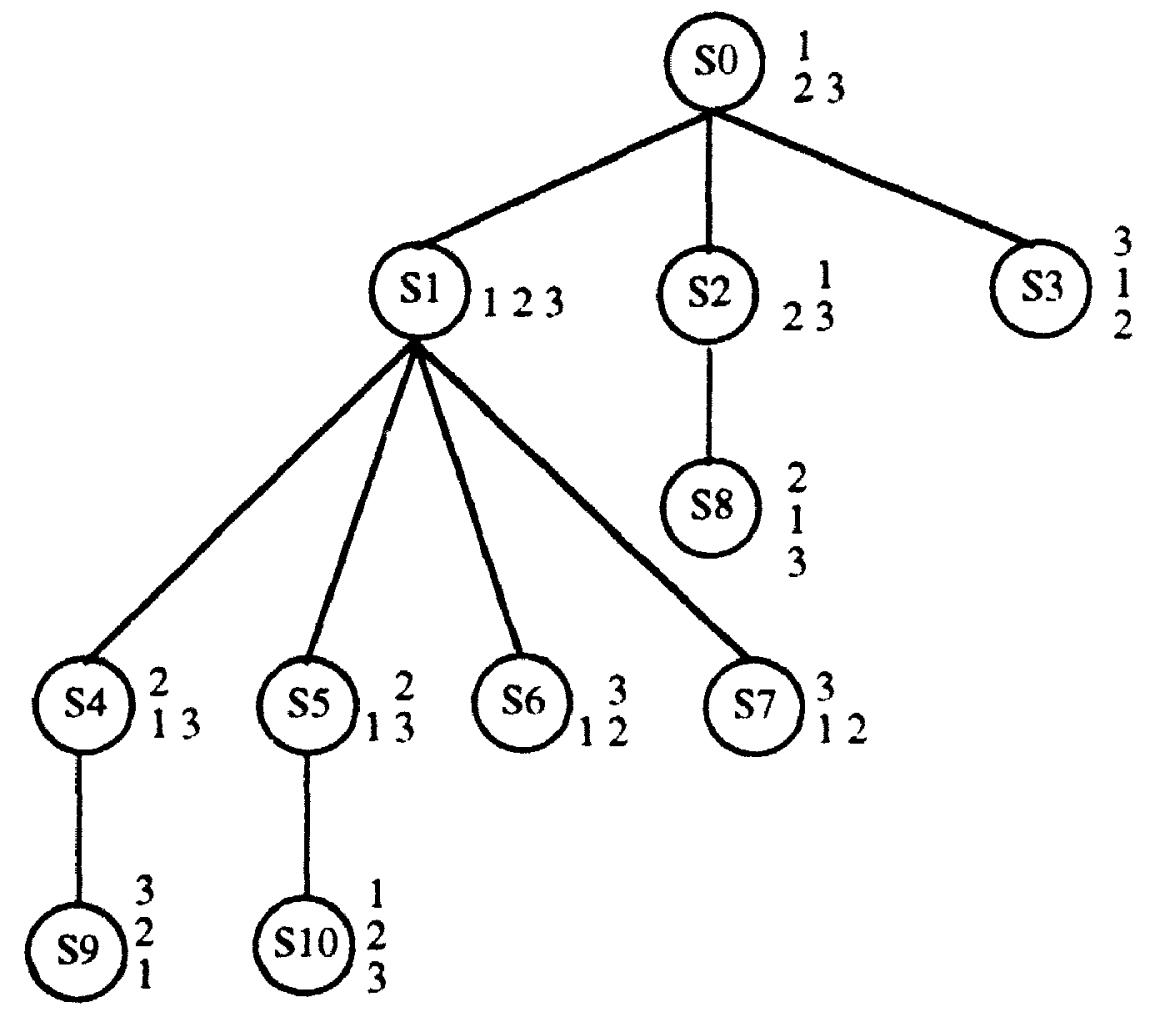

Figure 3.24 The state-space graph of the block rearrangement problem 
The nodes are labelled $S_{0}$ through $S_{10}$. Each node is obtained by the ordering of the three blocks. For example, node $S_{1}$ corresponds to the successor state of $S_{0}$ reached by "MOVE block 1 to the table". The nodes are generated and expanded in the order given by their labels. When the algorithm terminates, finding $S_{10}$ to be the goal, the list of expanded nodes contains $S_{0}$ through $S_{5}$, and the OPEN list still contains $S_{6}$ through $S_{10}$

\subsubsection{Implementing The Breadth-first Search in Parallel}

To accomplish the implementation of the breadth-first search in parallel, note that at each level of the search, tasks are created that correspond to every node of the state-space graph. We assume that these tasks can be executed independently. Consider the above example. An initial task is created in correspondence to the node $\mathbf{S}_{0}$. Then tasks that correspond to $S_{1}, S_{2}$ and $S_{3}$ are created and placed on the task queue. The search for the state-space graph continues until the final state is reached. Once the final state is reached, the algorithm enters the wait \& abort stage where the tasks that do not yield the results will be aborted. The implementation of the breadth-first search is similar to the divide-andconquer algorithms, except that the merge tasks in this case also perform task abortion when needed. A task system which gives a high-level view of the structure of the parallel breath-first search is shown in Figure 3.25. Since this class of programs are essentially performing the state-space search on a graph, from now they are referred to as state-space search. 


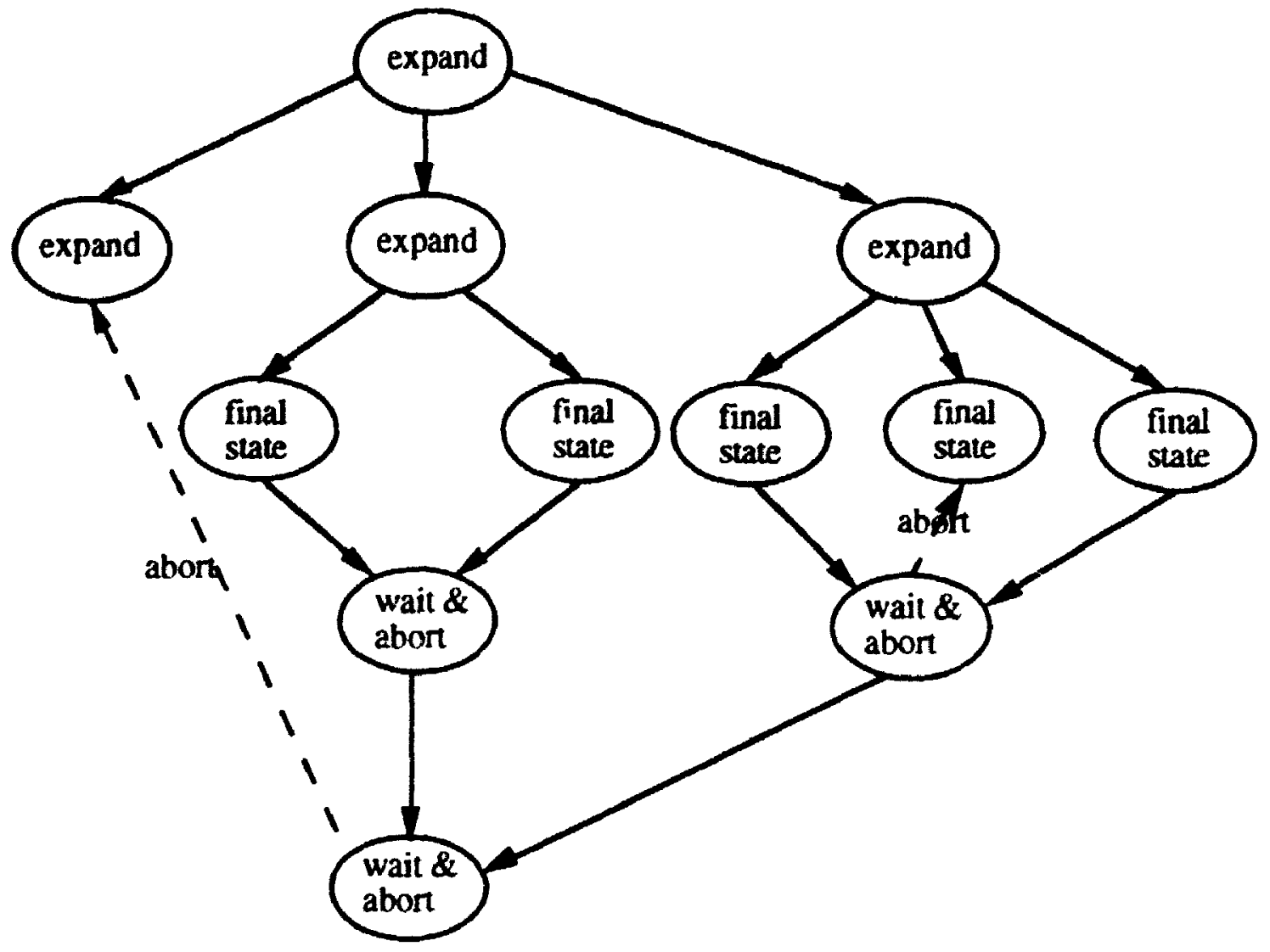

Figure 3.25 A task graph of the parallel breath-first search

\subsection{Parameters of The Abstract Model}

This section presented an abstract model for parallel programs. The abstract model is a set of input parameters for the simulation model. They are listed in Table 3.1.

\begin{tabular}{|c|c|}
\hline Parameters & Description \\
\hline$m p$ & Maximum Parallelism \\
\hline$C_{V_{m p}}$ & Coefticient Variation of Maximum Parallelism \\
\hline num phases & number of parallel phases \\
\hline$d$ & Mean Service Demand \\
\hline$C V_{d}$ & Coefficient Variation of Service Demand \\
\hline$t$ & Probability of Task abortion \\
\hline
\end{tabular}

Table 3.1 Input parameters for the simulation model (an abstract model) 


\section{Maximum Parallelism (mp)}

The maximum parallelism of a parallel program refers to its maximum number of tasks that can be run in parallel, if an unlimited number of processors is provided. Since we assume that the tasks in a fork-and-join job can be executed independently, its maximum parallelism is always equal to the number of tasks the job has. For the divide-and-conquer job model, the maximum parallelism is achieved when the job is in its work stage. For the matrix factorization, the maximum parallelism is at the second stage of the parallel program, where independent tasks are created to work on each column of the matrix. However, as the execution progress is, the number of parallel tasks decreases as the number of columns to be solved decreases. For the parallel state-space search jobs, maximum parallelism is reached when the search enters the final state.

\section{Number of parallel phases (num_phases)}

This parameter specifies the number of parallel phases in the input jobs. For the fork-and-join job model, this parameter represents the number of parallel phases which are separated by a synchronization point in the program. For the divide-andconquer job model, this parameter represents the sum of the number of divide phases, work phases and merge phases. For tie gaussian elimination job model. this parameter represents the number of iterations in the program. Finally for the state-space search job model, this parameters corresponds is the sum of the number of expand phases, final state phases and the synchronization and abortion phases.

\section{Mean Service Demand (d)}

There are two approaches to model the service demand of a parallel program. One is to compute the overall service demand of a parallel program and distribute the overall service demand among the tasks of the program. We can 
compute the service demand of each task of a parallel program and sum them up to get the overall service demand. The workload created from the first approach is called uncorrelated because the overall service demand of a parallel program is independent of the maximum parallelism. On the other hand, the model based on the second approach is called correlated because the overall service demand of a job is directly proportional to the maximum parallelism.

For the uncorrelated model, the parameter $d$ represents the mean service demand of a parallel program. For example, if it is set to 20.0, then, on average, the parallel program will impose 20.0 units of service demand onto the system.

For the correlated model, $d$ is the mean service demand of the tasks in the parallel programs. For example, if $d$ is equal to 1.0 , then on average, every job has a mean task service time equal to 1.0. The mean task service time is the sum of the service time of the tasks divided by the number of tasks in the job.

\section{Coefficient of Variation Service Demand $\left(C V_{d}\right)$}

$C V_{d}$ is the coefficient of variation of service demand of parallel programs, if the first asproach is used. It is the coefficient of variation of service demand of the tasks, if the second approach is used.

\section{Probability of Task Abortion}

The probability of task abortion is only used by the state-space search job model. It is used to decide whether a task should be aborted by the wait \& abort tasks.

These parameters along with the job structure defines the workload. The four types of job structures considered in the thesis are fork-and-join (Figure 3.3), divide-and- 
conquer (Figure 3.11), Gaussian Elimination (Figure 3.20), state-space search (Figure 3.25).

Summary

This chapter presented a survey of the parallel implementations of four classes of algorithms. Examples of the implementation were described. An abstract model, a set of input parameters for our simulation, was presented at the end of this chapter. The model was capable of specifying parallel programs studied in this chapter. 


\section{CHAPTER IV}

\section{SCHEDULING POLICIES}

This chapter discusses the scheduling policies used in the simulation experiments: These are the round robin job policy (RRjob) [Leutenegger \& Vernon 90], the dynamic partition policy $(D P)$ [Zahorjan \& McCann 90] and the coscheduling policy (CoSched) [Leutenegger \& Vernon 90]. [Ousterhout 82]. Each of these policies has its advantages and disadvantages. In addition, we will also evaluate the performance of the preemptive smallest cumulative demand first policy (PSCDF). PSCDF provides good performance and can serve as a benchmark for comparison. $R R j o b$ aims at providing equal shares of the processing power to the jobs in the system, regardless of their number of tasks. This is done by varying the quantum size for each job in the system. Since RRjob provides equal allocation per job, it results in good performance in multiprocessor systems [Leutenegger $\&$ Vernon 90 ]. $D P$ has the same objective as $R R j o b$ in that it tries to make the jobs share the processing power equally [Leutenegger \& Vernon 90]. In addition, $D P$ also aims at minimizing the number of context switches and reacting dynamically to the changes in the job's parallelism. If the tasks of the same job require coordination among them, it would be better if these tasks can be scheduled to run at the same time. The CoSched policy is designed in light of this requirement [Leutenegger \& Vernon 90], [Ousterhout 82]. Even though the Cosched policy is shown to perform worse than the DP and RRjob policies, the Cosched policies is considered in this thesis because of the presence of synchronization in the workload. 
In the subsequent sections, the four scheduling policies are described in detail and show the additional requirements of these policies in order to schedule the parallel jobs that are considered in this thesis.

\subsection{The CoScheduling Policy}

It is assumed that a linked list is used to implement the task queue. A processor window of size equal to the number of processors in the system is moved along the linked list. When a joh arrives into the system, its tasks are appended to the end of the linked list.

At each quantum, tasks located in the processor window are scheduled to run. Some tasks may have service demand less than a quantum. When these small tasks are completed, their processors remain idle until the end of this quantum. All processors do the context switch at the same time. Example runs of a system with four processors are given as follows. 1

\section{Example 1:}

1st quantum: A1 A2 A3

2nd quantum: A1 A2 A3 B1 B2 B3 B4

3rd quantum: AlA2]A3 B1 B2 B3 B4 CIC2

4th quantum A1 A2 A3 B1 B2 B3 B4 C1 C2

5th quantum: A1 A2 A3 $\mathrm{B1B}$ B B B $4 \mathrm{Cl} \mathrm{C2}$

In example 1, the system begins with only job $A$ which consists of tasks $A_{1}, A_{2}$ and $A_{3}$. In the first quantum, the scheduling window is placed on the tasks of job $A$.

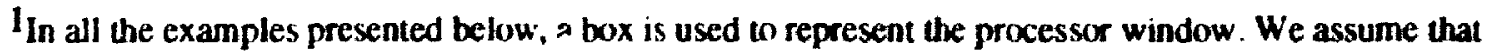
there are 4 processors in the system. Capital letters are used to represent a job (or program). The tasks $i$ of job $A$ is represented by $A i$.
} 
Hence, the tasks $A_{1}, A_{2}$ and $A_{3}$ are scheduled to run on these processors. Since all the tasks of job $A$ have been scheduled at the first quantum, in the second quantum the processor window is moved to job $B$. In the third quantum, tasks of job $C$ are scheduled. Since job $\mathrm{C}$ has only two tasks, the remaining processors in the window will be used to execute the tasks of job $A$. In the $4^{\text {th }}$ quantum, tasks of job $A$ is scheduled because not all the tasks of job A were scheduled in the previous quantum; and this shows the effect of a job that is not being completely coscheduled. A job is completely coscheduled, if all its tasks have been scheduled to run in the previous quantum. At the end of each quantum, the processor window is moved along the linked list until the head of the window is at the first task of the job that is not completely coscheduled in the previous quantum.

However, this definition cannot be applied to the fork-and-join parallel jobs when the number of tasks $P$ is greater than the number of processors $N$ in the system. For the fork-and-join parallel jobs consisting of $P>N$ tasks, it is impossible to schedule all the tasks of the job in one quantum. In this case, the job is completely coscheduled through the schedules in the previous consecutive quanta. An example scheduling sequence of forkand-join parallel jobs of this type is given below.

\section{Example 2:}

1st quantum: A1 A2 A3 A4 A5 A6 A7

2nd quantum: A1 A2 A3 A4 A5 A6 A7 B1 B2 B3

3rd quantum: A1 42 A3 A4 A5 A6 A7 B1 B2B3

4th quantum: $\mathrm{A} 1 \mathrm{~A} 2 \mathrm{~A} 3 \mathrm{~A} 4 \mathrm{~A} 5 \mathrm{~A} 6 \mathrm{~A} 7 \mathrm{~B} 1 \mathrm{~B} 2 \mathrm{~B} 3$

From the above example, we see that job $\mathrm{A}$ has more tasks than the number of processors in the system. Thus, job $A$ is completely coscheduled if and only if all its tasks 
are scheduled $i$. two or more consecutive quanta. as in the case of quantum 1 and 2 , and quantum 3 and 4.

The hasic definition of complete coscheduling $m$ : $y$ not be app'irable to the job models where new tasks can he created dynamically, as the tasks of the same job may not be grouped together in the queue. Consider the scheduling sequence of the parallel divideand-conquer jobs that is giv $\longleftarrow n$ in example 3 .

\section{Example 3:}

1st quantum $\mathrm{A} \mid \mathrm{B}[\mathrm{C}] \mathrm{D}]$

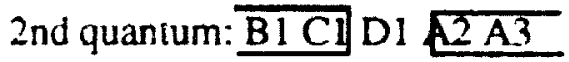

3rd quantum: $\mathrm{C} 1 \mathrm{D} 1 \mathrm{A2}$ A3 B2 B3

4th quantum: C1 D1 B3 A4 A5 B4B5 A6 A7

At the 4th quantum, the tasks of job $A$ and $B$ are interleaved and these jobs cannot he completely coscheduled, even though their number of tasks is less than the number of processors. Under these circumstances, a loose definition of complete coscheduling is adapted. A job is considered to be completely coscheduled. if a group of its tasks that have the same ancestor is completely coscheduled. Thus, in the ahove example. jobs A and B are both completely coscheduled by the fourth quantum. This definition does not violate the spirit of roscheduling, because only the processes that are created by the same task are required to syncironice with each other.

\subsection{The Preemptive Smallest Cumulative Demand First Policy}

A priority queve is used to implement this policy. When a new job arrives, the system will preempt processors that are currently running the tasks of jobs having larger cumulative demand than the new joh. Thus, jobs with small service demand have higher 
priority than those with large service demand. Since the service demand of the parallel divide-and-conquer jobs changes during their execution, due to task creation and termination, we use their total service demand as their job size. By total service demand, we mean the sum of the service demand of all the tasks of the given job. Their total service demand decreases when their tasks are completed. In reality, this policy cannot be implemented, as the total service demand of a job is not known in advance.

\subsection{The Round Robin Job Policy}

The RRjob policy was originally defined by [Leutenegger \& Vernon 90]. The goal of the RRjob policy is to provide equal allocation of processors to jobs temporally. This is done by giving jobs with a small number of tasks a longer time slice. In addition, RRjob is a dynamic scheduling policy in that the processor allocation changes dynamically.

A shared queue of parallel jobs is used to implement this policy. Each entry in the queue consists of a list of tasks of the job. Scheduling is done round robin on this queue. Tasks of the first job in the queue is scheduled to run, when there is at least one processor available. If there are $M$ processors available, where $M \leq P$ (the number of processors in the system) and the job at the front of the queue (call it job f) has $N$ tasks waiting for service, where $N \leq M$, these $N$ tasks are scheduled to run in the next quantum. If $N<M$, the remaining $M-N$ processors will be given to the next job on the queue. On the other hand, if $N \geq M$. only $M$ tasks will be scheduled and the remaining tasks will have to wait for the next quantum of job $\mathrm{f}$.

The quantum size of this policy varies from job to job and it depends on the number

of tasks the joh has. The quantum size is equal to $(P * q) / N$, with $q$ being the unit size for a quantum, if the number of tasks in the job is less than $P$; otherwise, the quantum size is equal to $g$. 
Since RRjob is a dynamic policy, scheduling is done when a new job arrives or a task finishes its execution. A formal description of this policy is shown below.

$$
\begin{aligned}
& P=\text { number of processors in the system } \\
& M=\text { number of idle processors } \\
& N_{3}=\text { number of tasks in job } i \\
& W_{1}=\text { number of waiting tasks in job } i
\end{aligned}
$$

Suppose that job $i$ is the job to be scheduled next and there are $M$ idle processors available.

\section{RRjoh}

WHILE ( processorsCount $>0) \& \&$ ( there exist a waiting task ))

Joh $\mathrm{i}$ is the joh at the head of the queue

$$
\text { IF }\left(W_{1} \geq P\right)
$$

Schedule the first $M$ tasks of job $\mathrm{i}$ with quantum size $=q$

ELSE IF $\left(\left(N_{1} \leq P\right) \& \&\left(W_{1} \leq M\right)\right)$

Schedule all the waiting tasks of Job $\mathrm{i}$

with quantum size $=\left(P^{*} q\right) / N_{1}$

$$
\text { IF }\left(W_{1} \leq M\right)
$$

Use RRjob to schedule the next job to run on the remaining

$$
M \text {-!" , processors }
$$

\section{ENDIF}

\section{ENDIF}

move the queue-head pointer to the one following joh $\mathrm{i}$

\section{END WHILE}

End RRjob

An example run of this policy is stated as follows. Suppose that the system has 4 jobs $A, B, C$ and $D$. Program $A$ has 4 lasks $A_{1}, A_{2}, A_{3}$ and $A_{4}$. Program $B$ has two tasks $B_{1}$ and $B_{2}$. Program $C$ has three tasks $C_{1}, C_{2}$ and $C_{3}$. Assuinic that the service demand of these tasks are large and $q=1.0$ time unit. There are 3 processors in the system, namely, $X, Y$ and $\mathrm{Z}$. 
The allocation of processors between time 0.0 to time 6.5 is shown in Figure 4.1. As job A has more tasks than the number of processors in the system, its first three tasks will get 1.0 time unit of the processing power. Once the quantum for job 1 is expired, processors will be switched to the next job in the queue, namely, job B. Since job B only has two tasks, its quantum size is equal to $3 * 1.0 / 2=1.5$. The remaining processor, namely, processor $Z$ is allocated to job 3 . As job 3 has 3 tasks, its quantum size is equal to 1.0. Programs in the system will be scheduled in this way as long as there is an idle processor and a task waiting for service. By varying the quantum size of the jobs, this policy tends to provide an equal share of processing power among all jobs.

If the parallel job is not fork and join, the number of tasks per job changes dynamically. In these cases, the current number of tasks in a job is used for computing the quantum size. 


\begin{tabular}{|c|c|c|c|}
\hline Time & $x$ & $Y$ & 2 \\
\hline $0.0-0.5$ & \multirow{2}{*}{ al } & \multirow{2}{*}{22} & \multirow[t]{2}{*}{$a^{3}$} \\
\hline $0.5-1.0$ & & & \\
\hline $1.0-1.5$ & \multirow{3}{*}{ bl } & \multirow{3}{*}{ b2 } & \multirow{2}{*}{ cl } \\
\hline $1.5-2.0$ & & & \\
\hline $2.0-2.5$ & & & \multirow{2}{*}{ al } \\
\hline $2.5-3.0$ & \multirow{2}{*}{ c3 } & \multirow{2}{*}{24} & \\
\hline $3.0-3.5$ & & & \multirow{2}{*}{ al } \\
\hline $3.5-4.0$ & \multirow{2}{*}{ a2 } & \multirow{3}{*}{ bl } & \\
\hline $4.0-4.5$ & & & \multirow{3}{*}{ b2 } \\
\hline $4.5-5.0$ & \multirow{2}{*}{$\mathrm{cl}$} & & \\
\hline $5.0-5.5$ & & \multirow{2}{*}{$c 2$} & \\
\hline $5.5-6.0$ & \multirow{2}{*}{ c3 } & & \multirow{2}{*}{ al } \\
\hline $60-65$ & & & \\
\hline
\end{tabular}

Figure 4.1: Processor allocation of the RRjob policy

\subsection{The Dynamic Policy}

The dynamic policy is a two level scheduling policy [Leutenegger 90] and [Zahorjan \& McCann 90]. At the upper level, processors are partitioned among the jobs in the system. At the lower level, the task dispatcher assigns processors to the parallel jobs. The task dispatcher may release processors when the parallel jobs do not have enough parallelism to utilize them. The actions of the processor allocator is described as follows.

When a job requests one or more processors, the following actions is taken

1. If there are idle processors, use them to fulfill the requests.

2. Otherwise, if the job making the requests is a new job, allocate it a single processor by preempting a processor from a joh that currently owns more than two processors. 
3. If any part of the requests for processors cannot be satisfied, it remains outstanding until either a processor becomes available for it or th: task dispatcher releases processors from other parallei jobs.

When a parallel job releases its processors, the processor allocator will search for jobs that have not yet been assigned a processor (i.e. the unsatisfied new arrivals) and assign a processor to each of these jobs. If there are more free processors after this allocations, scan the queue again, and allocate the rest of the processors on a FCFS basis.

When a processor finishes a task, the dispatcher will be invoked to either assign another task of the same job to the idle processor or hand it over to the processor allocator. The function of the dispatcher is described as follows.

4. If there is a newly arrived job that has not allocated a processor, give the processor to this job.

5. Otherwise, let $q_{j}$ be the number of job $j$ 's tasks waiting for processors. If $q_{j}=1$, the dispatcher simply dequeves the task and starts its execution.

6. If $q_{j}>1$ the task dispatcher will begin executing the first task in the queue

7. Finally, if $q_{j}=0$, the dispatcher will release its processors to the allocator. 


\section{CHAPTER V}

\section{SIMULATION EXPERIMENTS}

This chapter presents the results of the simulation experiments to evaluate the performance of scheduling policies introduced in the previous chapter. The job models given in chapter 3 are used. The output parameters are shown in Table 5.1.

\begin{tabular}{|c|c|}
\hline Parameters & Description \\
\hline$M R T$ & mean job response time \\
\hline$\rho$ & system utilization \\
\hline$n$ & mean number of tasks per job \\
\hline$C V_{n}$ & CV of number of tasks per job \\
\hline$d$ & mean job service demand \\
\hline$C V_{d}$ & CV of job service demand \\
\hline$s y n \_$delan & synchronization delay per job \\
\hline$c L x !$ & number of context switch per job \\
\hline
\end{tabular}

Table 5.1 The output parameters and their descriptions

All the simulations are parameterized for a system with $p=20$ processors. The quantum length $(Q)$ for the coscheduling and rjob policies is equal to 0.1 . The overhead for the context switch (ctxtOverhead) is assumed to be $1.5 \%$ of the quantum, that is 0.0015 . The overhead also applies to the pscdf and dynamic policies, when a processor is preempted from one task to another.

\subsection{The Impact of Job Structure on the Performance of Scheduling Policies}

The purpose of the first set of experiments is to compare the performance of the scheduling policies for different job models and utilization levels. In [Leutenegger 90], an experiment using the fork-and-inin model was conducted. In this section, the experiment is 
repeated over different job models to see whether the performance is affected by the change.

To provide a fair comparison, the output values of the average number of tasks and the average total service demand of the jobs must have the same values for all the job models. We call these the control parameters of the simulation. This is done by setting appropriate values for the input parameters. The utilization level is varied by changing the arrival rate of the parallel jobs. If $U$ is the target system utilization, $D_{T}$ is the average total service demand of the jobs in the workload, and there are $P$ processors in the system, the arrival rate $\lambda=\frac{U P}{D_{T}}$.

Since there are eight workload models in our experiments, a vector with two components is used to represent the models. The first one shows whether the job model is correlated or uncorrelated by using the abbreviations Cor or UnCor. The second component shows the type of the job models: Fork-and-Join model (FJ). Divide-andconquer model (DC), Gaussian elimination model $(\mathrm{GE})^{1}$ and Breadth first search model (SSS). For example, correlated fork-and-join workload is represented by (Cor, FJ).

Two types of workload, defined in the previous chapter, are considered (correlated and uncorrelated). The default values of the uncorrelated and correlated workload are shown if Table 5.2 and Table 5.3.

\footnotetext{
${ }^{1}$ Since matrix factorization is in basic solving the Gaussian Elimmation problem, we use GE to represent the matrix factorization algorithms.
} 


\begin{tabular}{|c|c|c|c|c|}
\hline Input & \multicolumn{4}{|c|}{ Default Values } \\
Parameters & FJ & \multicolumn{1}{c|}{ DC } & GE & SSS \\
\hline$m p$ & 20.0 & 12.444 & 5.667 & 12.444 \\
\hline$C V_{m p}$ & 1.0 & 1.0 & 1.0 & 1.0 \\
\hline$d$ & 20.0 & 20.0 & 20.0 & 20.0 \\
\hline$C V_{d}$ & 5.0 & 5.0 & 5.0 & 5.0 \\
\hline$P_{T}$ & 0.0 & 0.0 & 0.0 & 0.5 \\
\hline
\end{tabular}

Table 5.2 Default values of the input parameters (uncorrelated workload)

(Impact of job models on the performance of scheduling policies)

\begin{tabular}{|c|c|c|c|c|}
\hline Inpul & \multicolumn{4}{|c|}{ Default Values } \\
Parameters & FJ & \multicolumn{1}{c|}{ DC } & GE & SSS \\
\hline$m p$ & 20.0 & 12.444 & 5.667 & 12.444 \\
\hline$C V_{m p}$ & 1.0 & 1.0 & 1.0 & 1.0 \\
\hline$d$ & 1.0 & 1.0 & 1.0 & 1.0 \\
\hline$C V_{d}$ & 5.0 & 5.0 & 5.0 & 5.0 \\
\hline$P_{T}$ & 0.0 & 0.0 & 0.0 & 0.5 \\
\hline
\end{tabular}

Table 5.3 Default values of the input parameters (correlated workload)

(Impact of job models on the performance of scheduling policies)

The simulation experiments were run with system utilizations of $0 \%, 40 \%, 60 \%$, $70 \%, 80 \%$ and $90 \%$ for the uncorrelated and correlated workload for different job models. To reduce the effect of transients, the information is only collected after the measured throughput had reached $99.5 \%$ of the arrival rate. The batch method is then used to compute the confidence interval (in our case, 30 batch runs were used for the results reported here and each batch contains more than $50000 \mathrm{jcbs}$ ). This method gives $95 \%$ confidence intervals that were less than $1 \%$ of the mean response times when the system utilization is low to moderate and less than $20 \%$ for high utilization level (for example 90\%). This technique is also applied for all the experiments described in this chapter. 


\subsubsection{Results of the Fork-and-Join Job Model}

Figures 5.1 and 5.2 present the results of the fork-and-join model for the correlated and uncorrelated workload. The results shown here are consistent with the ones reported in [Leutenegger 90].

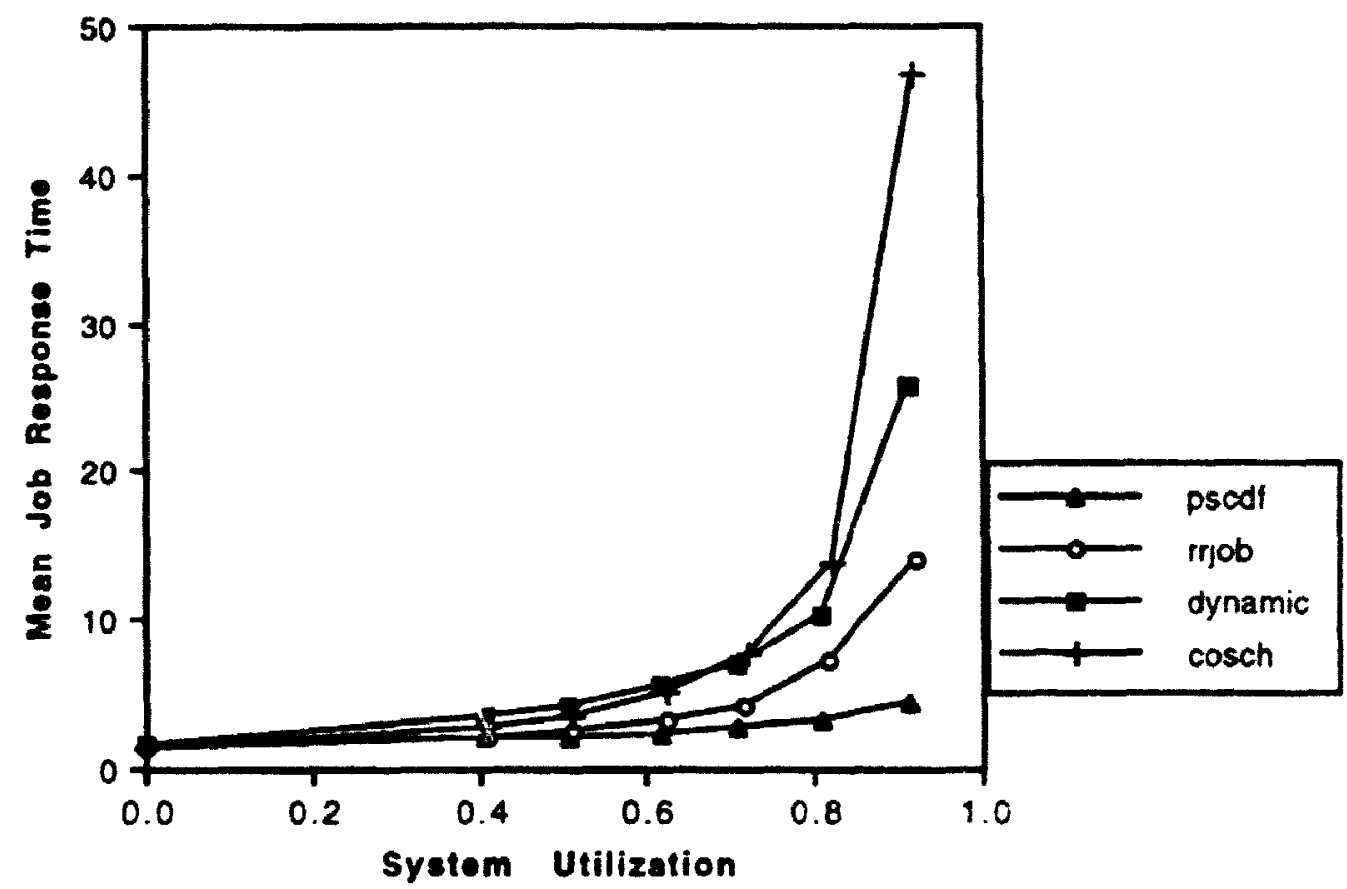

Figure 5.1 Performance of scheduling policies for the (Cor,FJ) model 


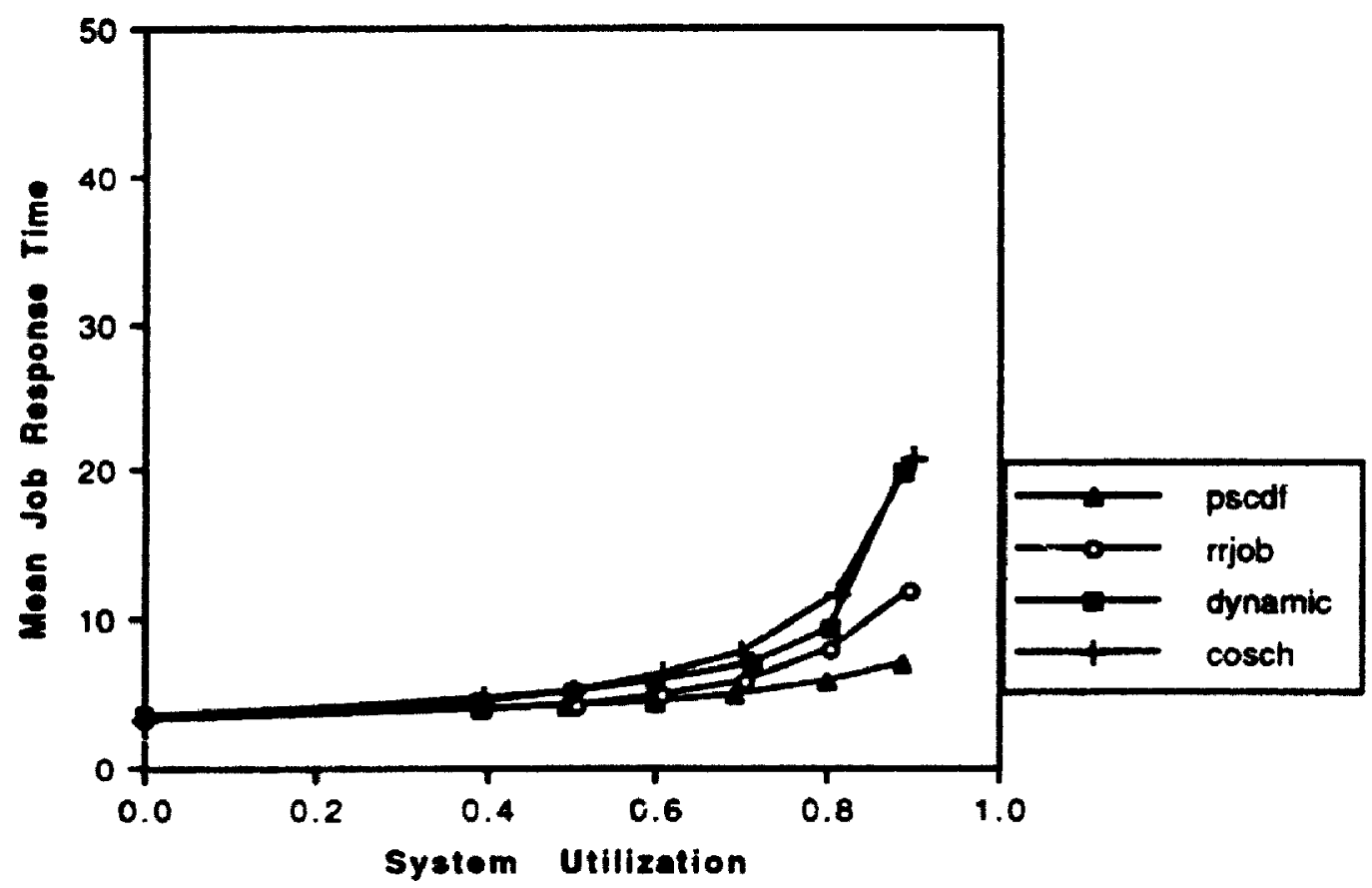

Figure 5.2 Perforn ance of scheduling policies for the (UnCor,FJ) model

Since rrjob provides the jobs with an equal share of the processing power, large jobs (in terms of total job service demand) do not dominate small jobs. This results in a shorter average response time for small jobs and therefore provides better overall job response time. On the other hand. the coscheduling policy performs poorly because it gives large jobs a larger share of processing power.

Dynamic policy allocates processors to jobs on a first-come-first-served basis. It does not adapt well to balance the processor allocation. Hence, fair allocation of processors to parallel jobs cannot be made at low utilization level. The problem becomes serious if the workload is correlated. Assume that the system has six processors. Consider the following sequence of jobs in the queue, where each task is assumed to require 2 time units of service. 
At time 0: $A_{1} A_{2}$

$\begin{array}{llllll}\text { Time } & P_{1} & P_{2} & P_{3} & P_{4} & P_{5}\end{array}$

Al time 1: $A_{1} A_{2} B_{1} B_{2} B_{3} B_{4} B_{5} B_{6} B_{7} B_{8} B_{9} B_{10}$

\begin{tabular}{lllllll} 
Time & $P_{1}$ & $P_{2}$ & $P_{3}$ & $P_{4}$ & $P_{5}$ & $P_{6}$ \\
\hline $0-1$ & $A_{1}$ & $A_{2}$ & & & & \\
$1-2$ & $A_{1}$ & $A_{2}$ & $B_{1}$ & $B_{2}$ & $B_{3}$ & $B_{4}$
\end{tabular}

Al time 2: $A_{1} A_{2} B_{1} B_{2} B_{3} B_{4} B_{5} B_{6} B_{7} B_{g} B_{9} B_{10} C_{1} C_{2}$

\begin{tabular}{|c|c|c|c|c|c|c|}
\hline Time & $P_{1}$ & $\mathbf{P}_{2}$ & $\frac{\operatorname{esso}}{P_{3}}$ & $P_{4}$ & $P_{5}$ & $P_{6}$ \\
\hline 0.1 & $A_{1}$ & $A_{2}$ & & & & \\
\hline 1.2 & $\mathbf{A}_{1}$ & $A_{2}$ & $B_{1}$ & $\mathrm{~B}_{2}$ & $B_{3}$ & \\
\hline $2-3$ & $C_{1}$ & $\mathrm{~B}_{5}$ & $\mathbf{B}_{1}$ & $\mathrm{~B}_{2}$ & $\mathbf{B}_{3}$ & \\
\hline
\end{tabular}

At ume 3: $B_{5} B_{6} B_{7} B_{8} B_{9} B_{10} C_{1} C_{2}$

\begin{tabular}{lllllll} 
Time & $\mathrm{P}_{1}$ & $\mathrm{P}_{2}$ & $\begin{array}{c}\text { Processors } \\
\mathbf{P}_{3}\end{array}$ & $\mathbf{P}_{4}$ & $\mathrm{P}_{5}$ & $\mathbf{P}_{6}$ \\
\hdashline $0-1$ & $\mathrm{~A}_{1}$ & $\mathrm{~A}_{2}$ & & & & \\
$1-2$ & $\mathrm{~A}_{1}$ & $\mathrm{~A}_{2}$ & $\mathrm{~B}_{1}$ & $\mathrm{~B}_{2}$ & $\mathrm{~B}_{3}$ & $\mathrm{~B}_{4}$ \\
$2-3$ & $\mathrm{C}_{1}$ & $\mathrm{~B}_{5}$ & $\mathrm{~B}_{1}$ & $\mathbf{2}$ & $\mathrm{B}_{3}$ & $\mathrm{~B}_{4}$ \\
$3-4$ & $\mathrm{C}_{1}$ & $\mathrm{~B}_{5}$ & $\mathrm{~B}_{6}$ & $\mathrm{~B}_{7}$ & $\mathrm{~B}_{8}$ & $\mathrm{~B}_{9}$
\end{tabular}


At time 4: $B_{5} B_{6} B_{7} B_{8} B_{9} B_{10} C_{1} C_{2} D_{1} D_{2}$

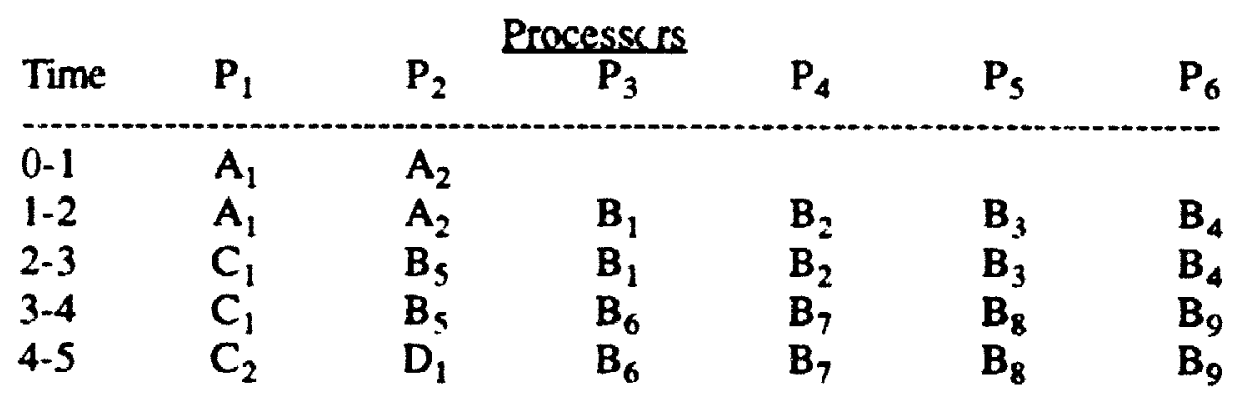

It can be seen from the above example that more processors are allocated to job $\mathbf{B}$, as it has more tasks than the other jobs. At low utilization level, where the multiprogramming level is low; the jobs with large number of tasks tend to get more processors allocated than those with smailet number of tasks. In the above example. job B tends to get more processors than the other jobs. It can be seen from Figure 5.2 that at low utilization levels, say hetween $0 \%$ to $70 \%$. the dynamic policy performs worse than coscheduling. However. as the utilization level gets higher than $70 \%$ the dynamic policy starts performing better than coscheduling. An increase in utilization means an increase in the number of jobs in the system. As a result of this, more jobs are run in parallel. This leads to a more equal distribution of processing power and a better performance of the dynamic policy.

\subsubsection{Results of the Divide-and-Conquer Job Model}

Results of the divide-and-conquer job model are presented in Figures 5.3 and 5.4. 


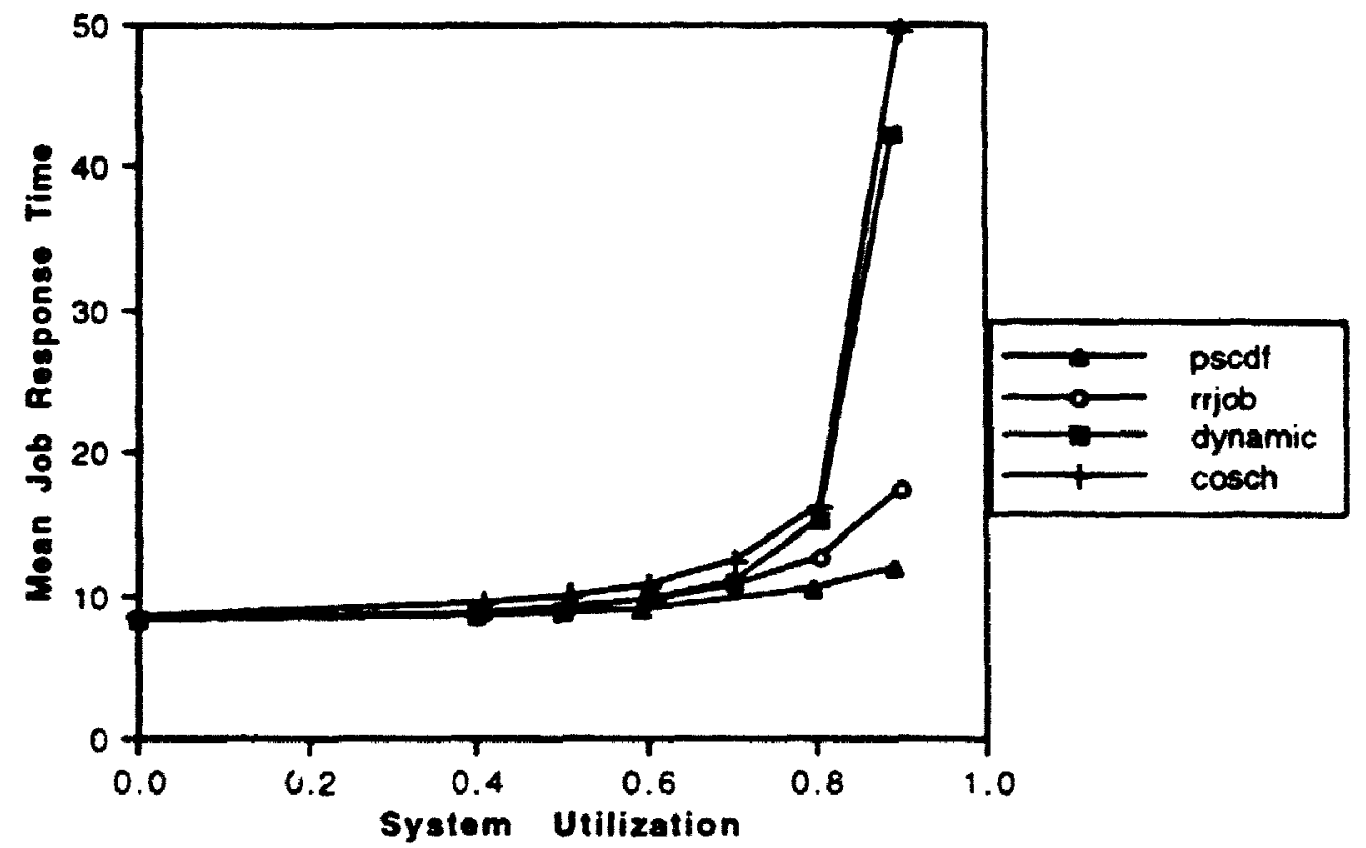

Figure 5.3 Performance of scheduling policies for the (Cor, DC) model

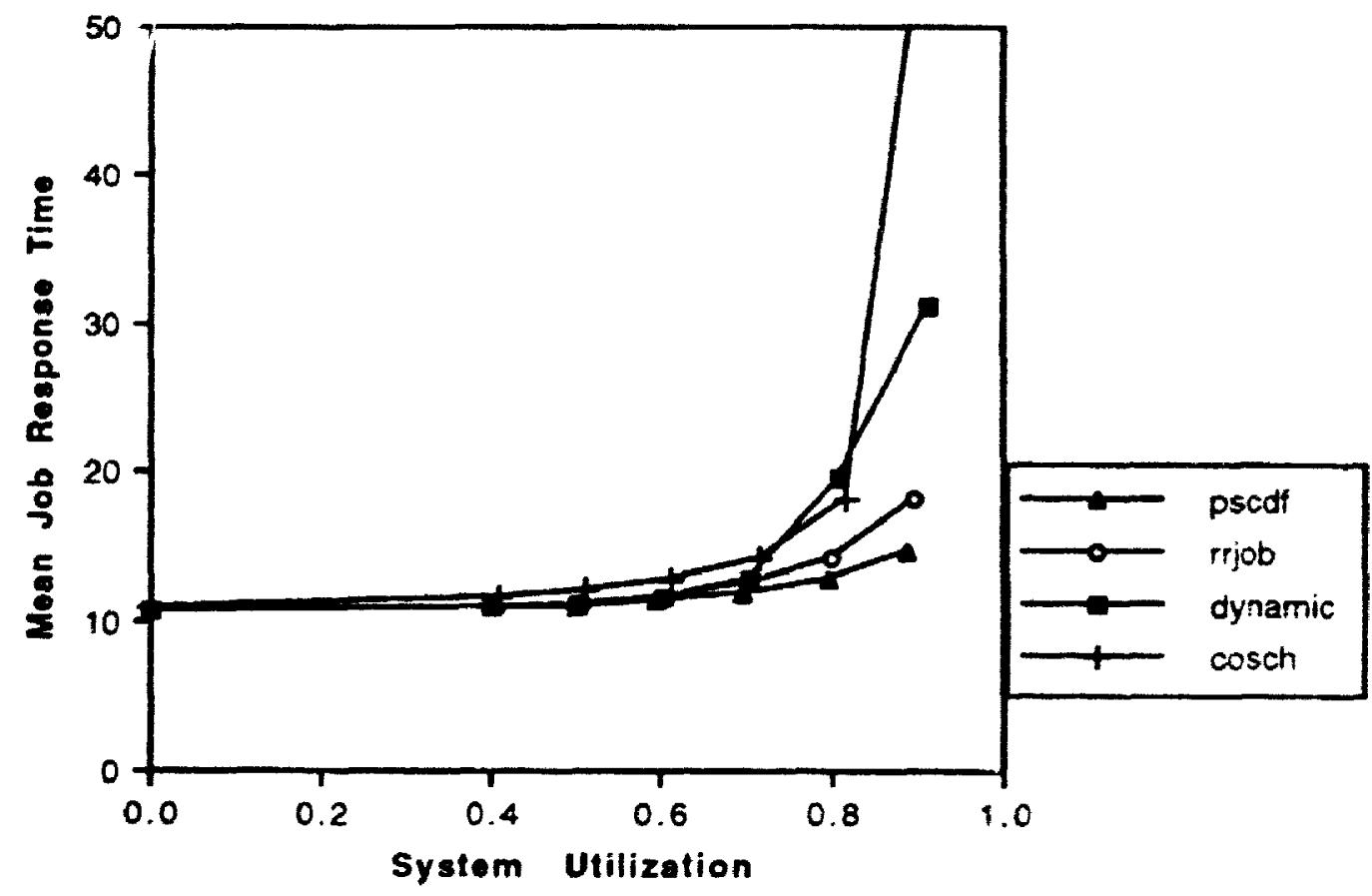

Figure 5.4 Performance of scheduling policies for the (UnCor,DC) model

Due to the dynamic nature of the job model, processors will be released hy parallel jobs during their execution. Thus, the processor allocation is balanced by the dynamic 
change of job parallelism and the performance of dynamic resembles that of the rijoh. Coscheduling performs poorly compared to the other two policies mainly because jobs with a larger number of tasks get a larger fraction of processing power than the jobs with a small number of task, thus increasing the waiting times of the small jobs.

\subsubsection{Results of the Gaussian Elimination Job Model}

Figures 5.5 and 5.6 prese it the results of the Gauscian Elimination job model.

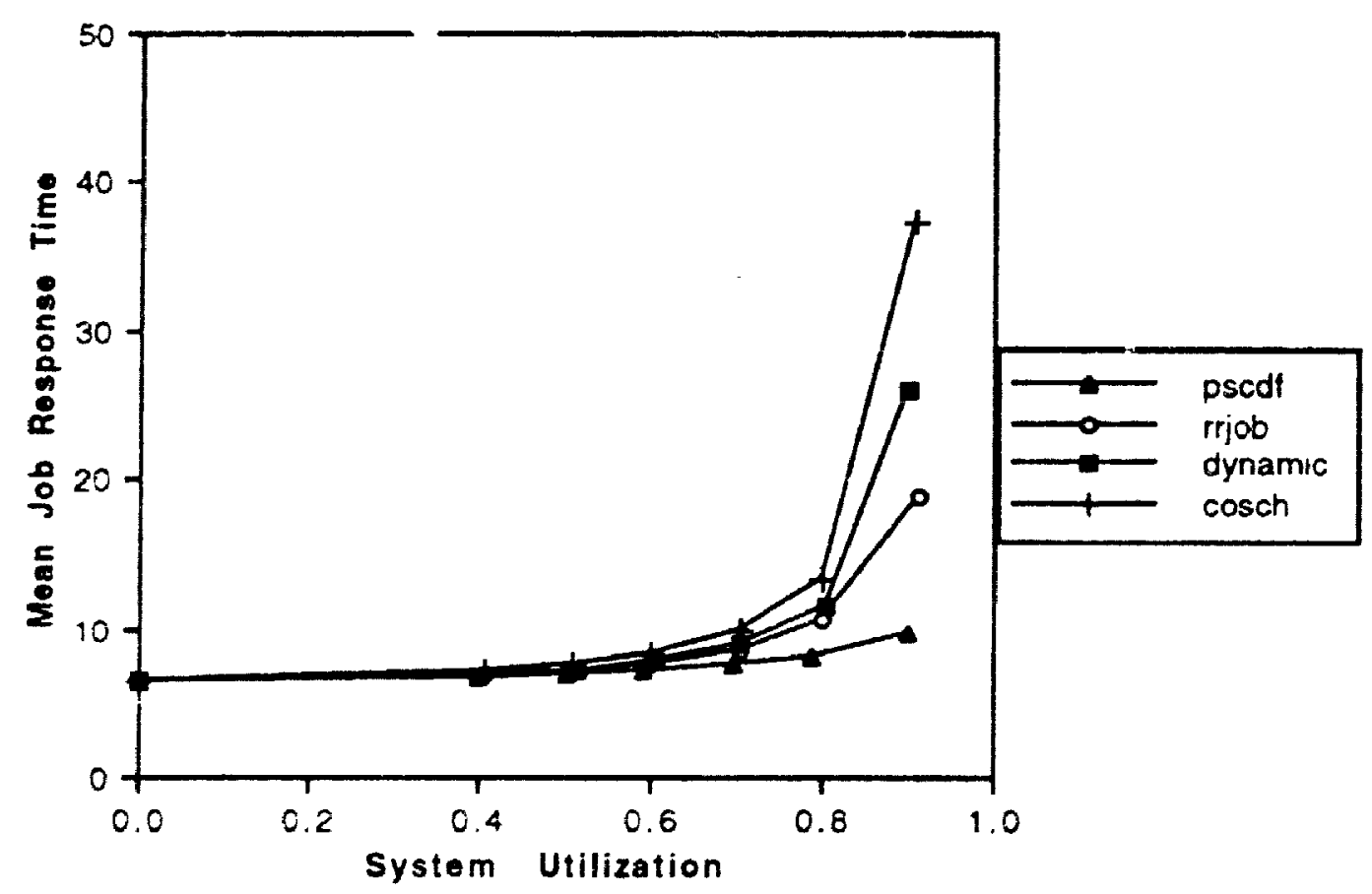

Figure 5.5 Performance of scheduling policies for the (Cor, GE) model 


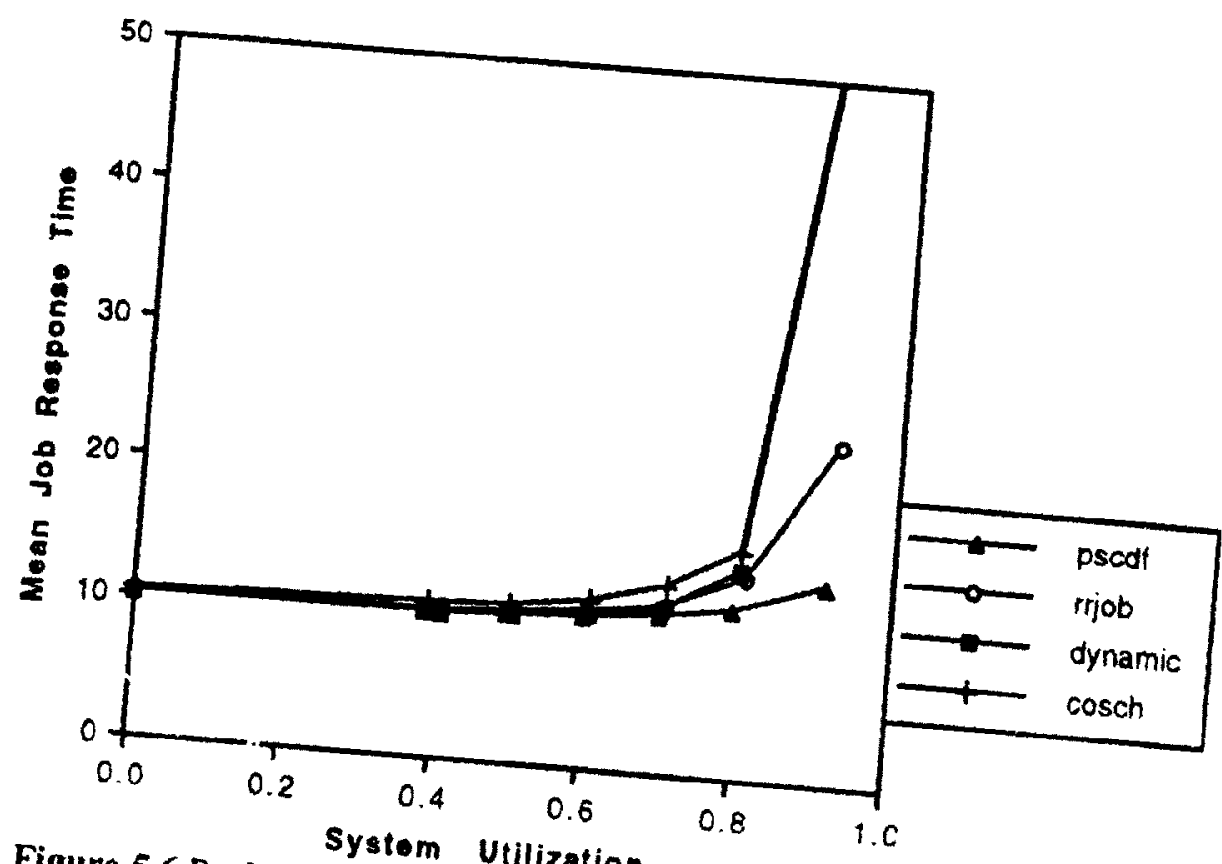

Figure 5.6 Performance sks at each parallel phase of the GE job model. it to finish in a single quantum of the rrjob. As a $s$ in longer job execution times and significantly the rrjob performs very similar to the dynamic

\subsubsection{Results of the Breadth First Search Job Model}

Figure 5.7 and 5.8 present the results for the breadth-first search job model.

\footnotetext{
${ }^{2} A$ pivot lask is the task for computing th" pivot column which is used to modify the other columns of the
matrix. Its execution is larger than the other tasks in the same parallel phase
} 


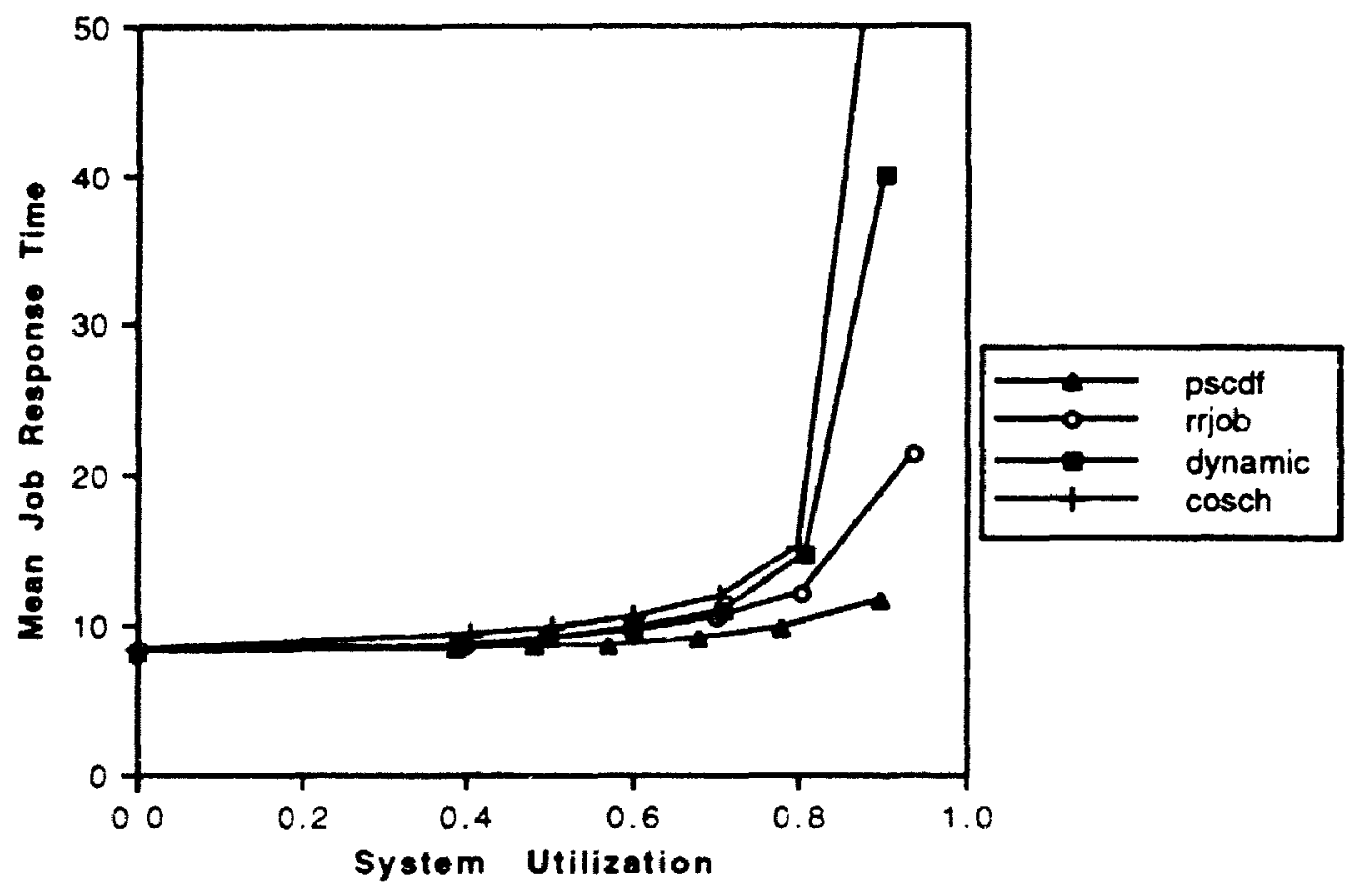

Figure 5.7 Performance of scheduling policies for the (Cor,SSS) model

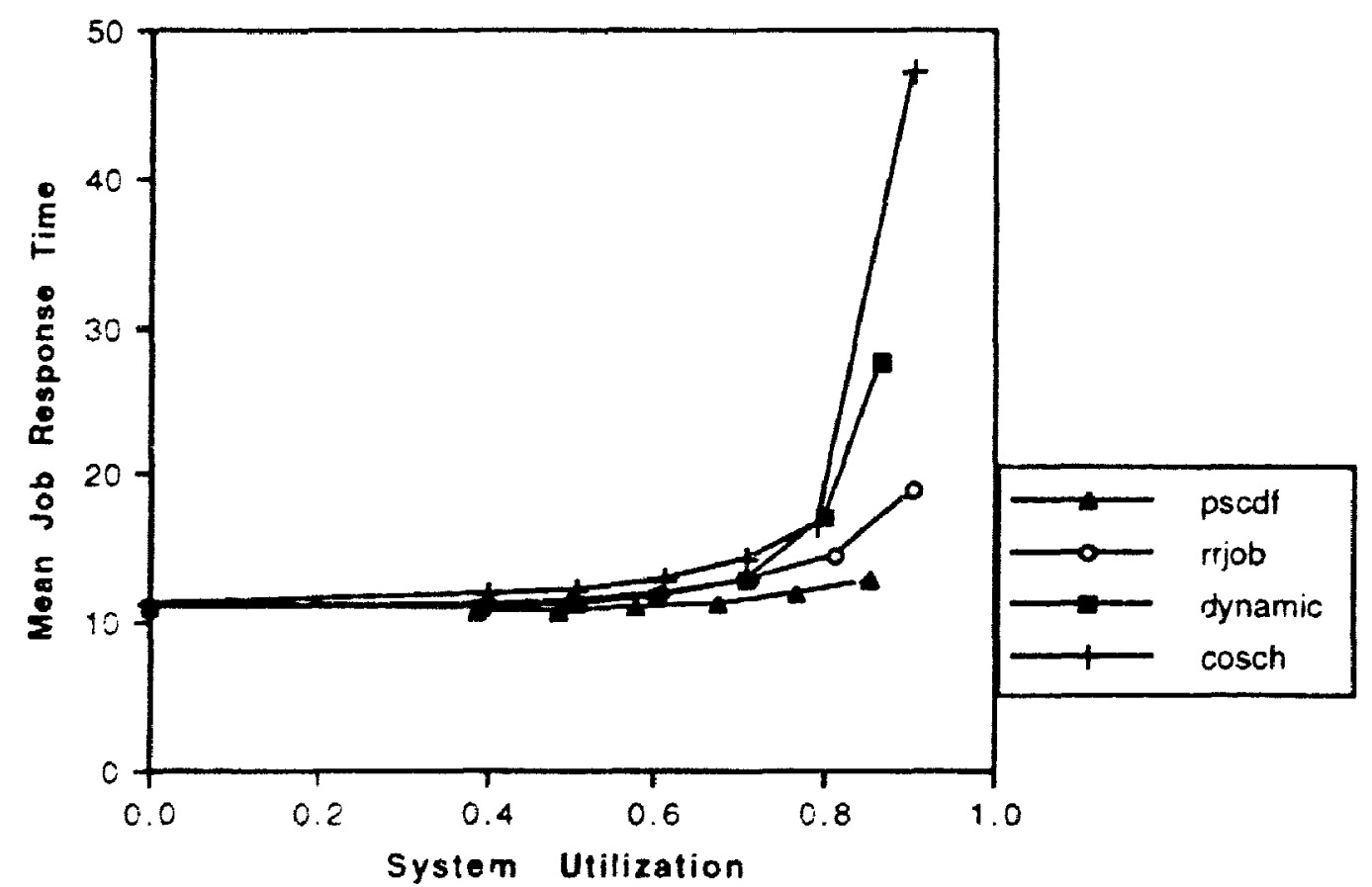

Figure 5.8 Performance of scheduling policies for the (UnCor,SSS) model

The difference hetween the fork-and-join and divide-and-conque models is the additional synchronization delay introduced by the divide and merge phases of the parallel 
jobs. For the SSS job model, the synchronization delay induced by the merge tasks are reduced by having task abortion. This is due to the termination of the aborted tasks. The divide-and-conquer job model can be treated as a SSS model without task abortion (i.e. probability of task abortion $p=0.0$ ). Note that, at the other extreme, when $p=1.0$, there is no synchronization in the SSS job model because the other task is always aborted.

Figures 5.7 and 5.8 show the results of the task abortion on the performance of scheduling policies. The value of $p$ in this experiment is set equal to 0.5 , so that the synchronization time induced by task abortion is in between 0.0 to 1.0. Then, on the average, there will be a decrease in mean job response time for all the scheduling policies by increasing the probability of task abortion. This change of the response time can be seen hy comparing Figures 5.3 and 5.4 (within $p=1.0$ ) with Figures 5.7 and 5.8 (within $p=0.5$ ).

\subsubsection{Surnmary of The Results}

Based on the results of this set of experiments, the following observations can be made.

1. Changing the job structure only affects the performance of the dynamic policy

2. Coscheduling (a fixed-preemptive) policy performs poorly for all the job models

3. For the DC, GE and SSS job models, dynamic performs as good as the rrjot. up to $70 \%$ of the utilization

4. Performance of policies is not affected by the presence of task abortion 


\subsection{Sensitivity of the Performance to $C V_{d}$}

In this section, we study the effect of $C V_{d}$ on the performance of the scheduling policies. The input parameters for different job models are set such that the output parameters are $n=20.0, C V_{n}=1.0, d=20.0$ and $C V_{d}=1.0,2.0,3.0,4.0$ and 5.0. The utilization level is fixed at around $70 \%$ by setting $\lambda=0.7$. Table 5.4 and 5.5 show the default values of the input parameters for the correlated and uncorrelated workload.

\begin{tabular}{|c|c|c|c|c|}
\hline Input & \multicolumn{4}{|c|}{ Default Values } \\
Parameters & FJ & DC & GE & SSS \\
\hline$m p$ & 20.0 & 12.444 & 5.667 & 12.444 \\
\hline$C V_{m p}$ & 1.0 & 1.0 & 1.0 & 1.0 \\
\hline$d$ & 1.0 & 1.0 & 1.0 & 1.0 \\
\hline$C V_{d}$ & vary & vary & vary & vary \\
\hline$P_{T}$ & 0.0 & 0.0 & 0.0 & 0.5 \\
\hline \hline
\end{tabular}

Table 5.4 Default values of the input parameters (correlated workload)

(Sensitivity of the Performance to $C V_{d}$ )

\begin{tabular}{|c|c|c|c|c|}
\hline Input & \multicolumn{4}{|c|}{ Default Values } \\
Parameters & $\mathrm{FJ}$ & $\mathrm{DC}$ & $\mathrm{GE}$ & SSS \\
\hline$m p$ & 20.0 & 12.444 & 5.667 & 12.444 \\
\hline$C V_{m \nu}$ & 1.0 & 1.0 & 1.0 & 1.0 \\
\hline$d$ & 20.0 & 20.0 & 20.0 & 20.0 \\
\hline$C V_{d}$ & vary & vary & vary & vary \\
\hline$P_{T}$ & 0.0 & 0.0 & 0.0 & 0.5 \\
\hline
\end{tabular}

Table 5.5 Default values of the input parameters (correlated workload)

(Sensitivity of the Performance to $C V_{d}$ ) 


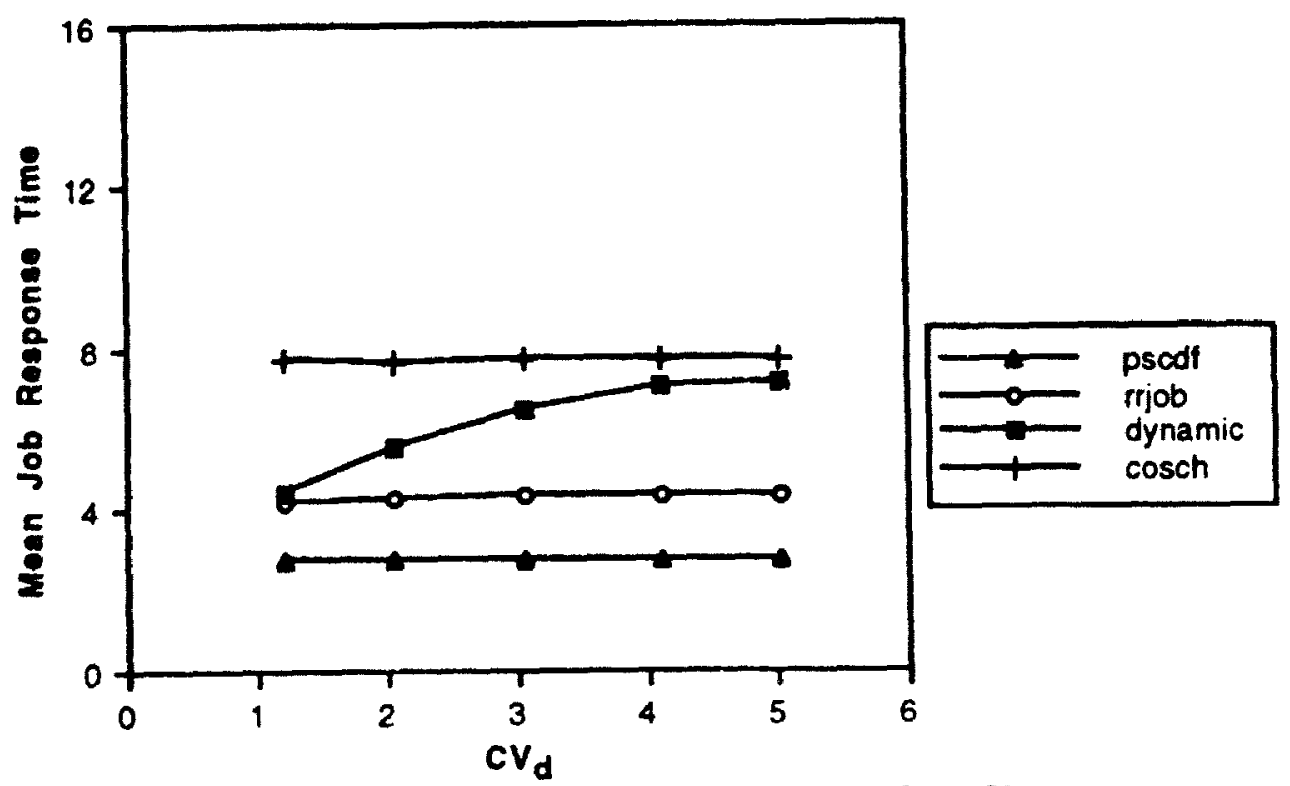

Figure 5.9 Sensitivity of performance to $C V_{d}$ (Cor, $\mathrm{FJ}$ )

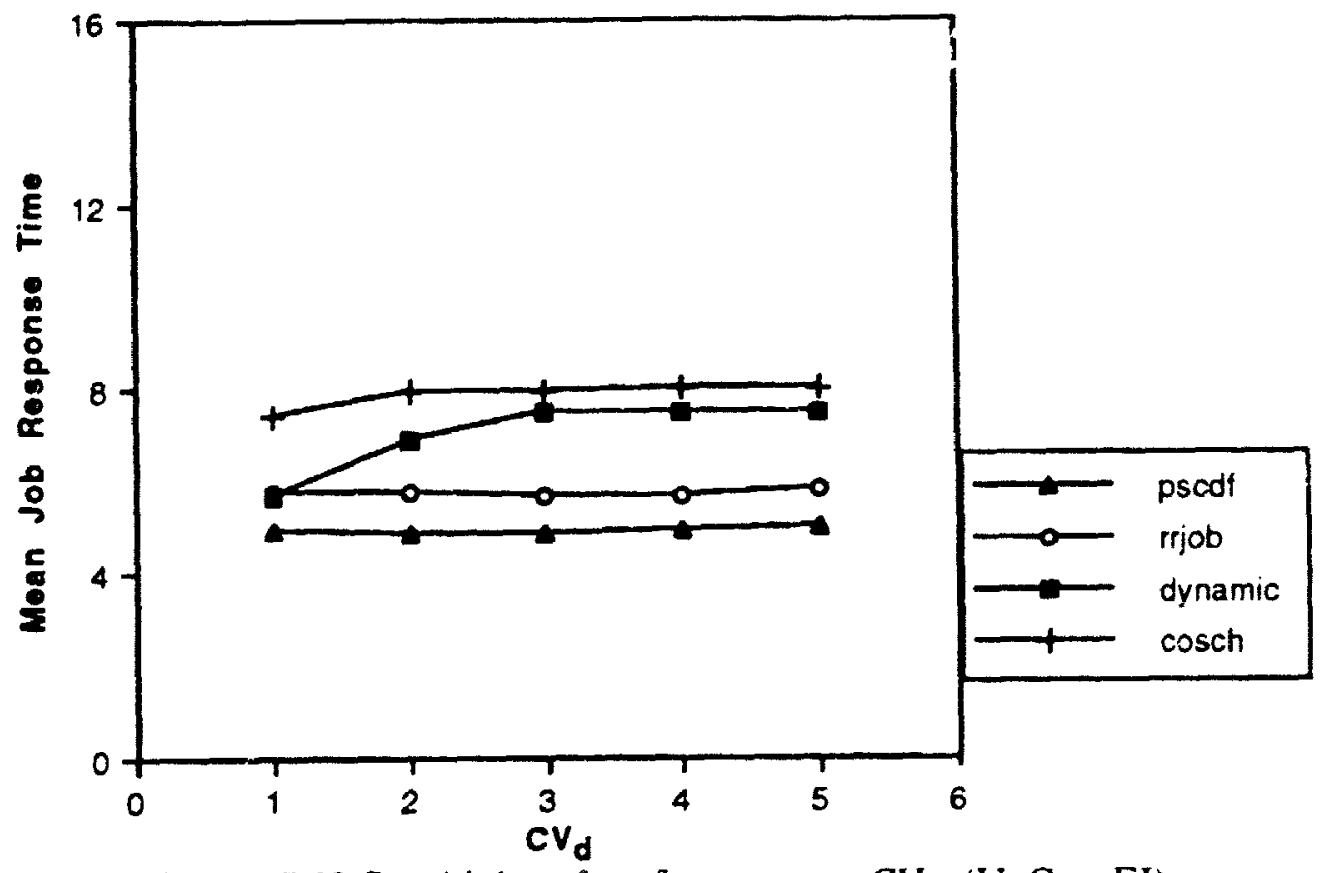

Figure 5.10 Sensitivity of performance to $C V_{d}$ (UnCor, $\mathrm{FJ}$ )

Figure 5.9 and 5.10 presents the results of the fork-and-join model. As $C V_{d}$ increases, the ratio between the service demand of large jobs to that of small johs increases. Dynanic policy is sensitive to the change of $C V_{d}$. An increase in $C V_{d}$ results in an increase 
in the variation of service demand among the jobs. Since the dynamic policy does not provide time-sharing of the processors to the jobs in the system, small jobs can be held up by some large jobs that had arrived earlier. This problem gets more serious as $C V_{d}$ increases. The other policies are insensitive to $C V_{d}$. rjob allocates the processing power based on job parallelism and time-sharing of the processors. An increase in $C V_{d}$ only increases the variation of job service demand and does not affect the processor allocation decision. For a similar reason, the cosched aling policy is also insensitive to $C V_{d}$. Since the pscdf policy always gives priority to the jobs that have smallest cumulative demand, varying the coefficient of variation of the service demand does not affect the performance of this policy.

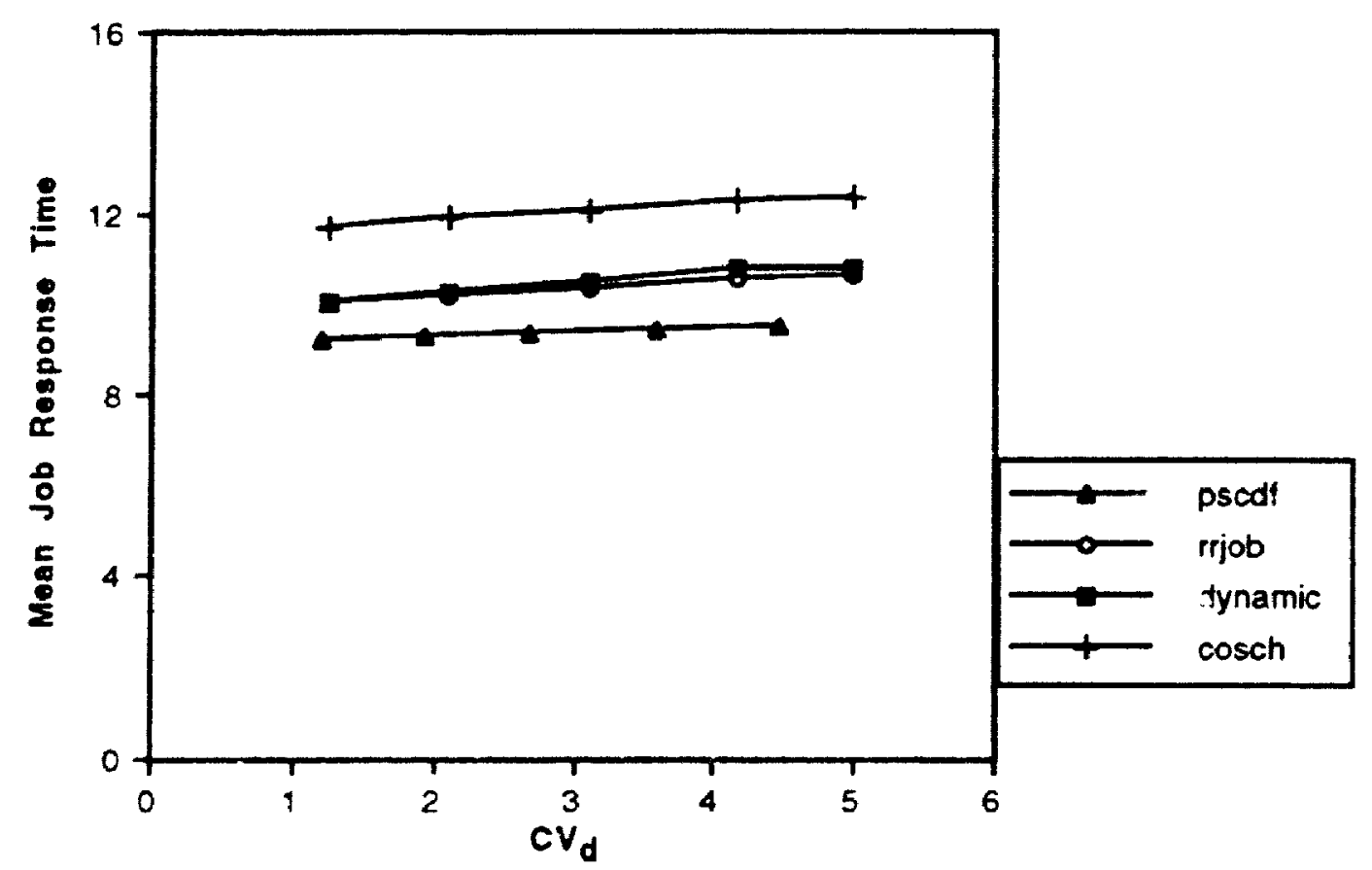

Figure 5.11 Sensitivity of performance to $C V_{d}$ (Cor, DC) 


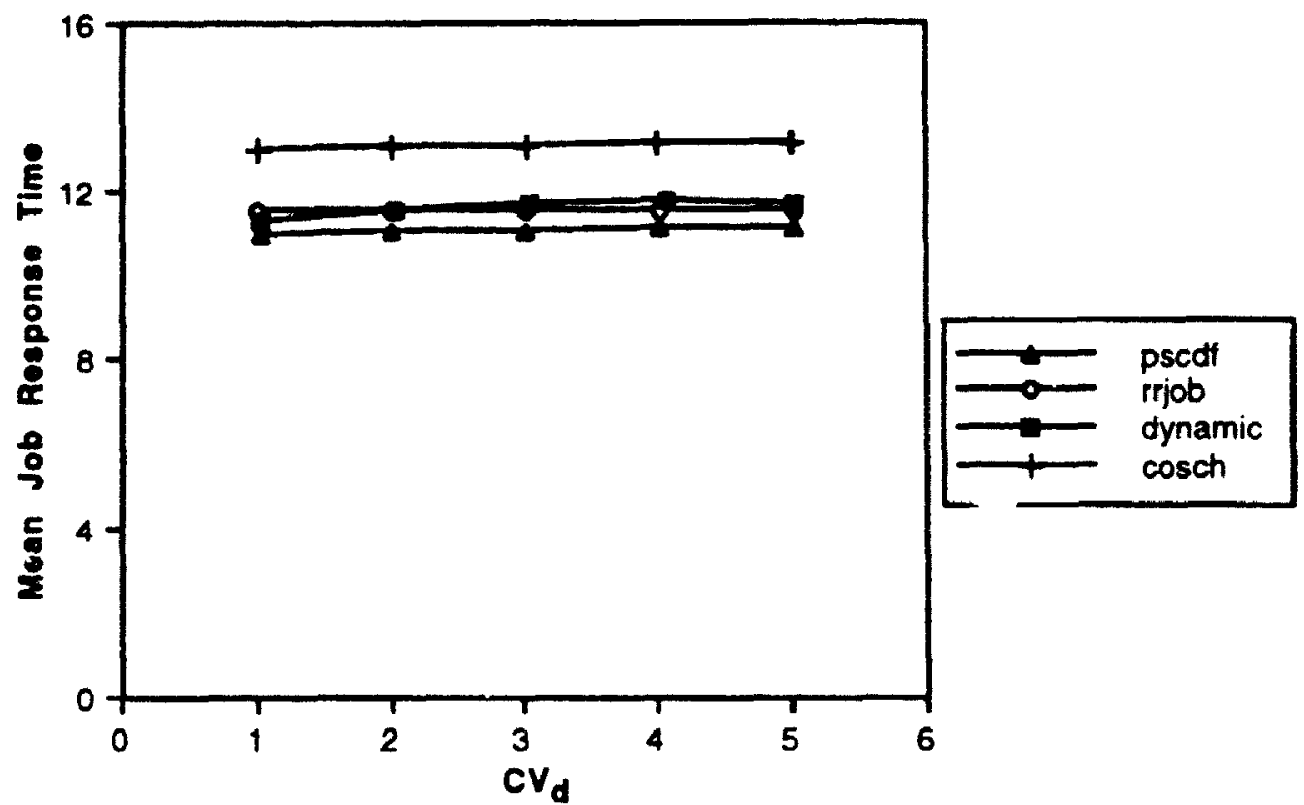

Figure 5.12 Performance of scheduling policies to $C V_{d}$ (UnCor, DC)

Figures 5.11 and 5.12 present the results for the divide-and-conquer job model. All four policies including the dynamic policy are all insensitive in $C V_{d}$. Due to the dynamic nature of this job model, processor reallocation can be used to meet the change in parallelism of a job. Thus, varying $C V_{d}$ does not degrade the performance on the dynamic policies. As mjob and Coscheduling allocate the processing power on a time sharing hasis, varying the $C V_{d}$ does not have effect on their performance.

Figure 5.13 through Figure 5.16 presents the results of the GE and SSS models. The results are similar to the ones for the divide-and-conquer model. 


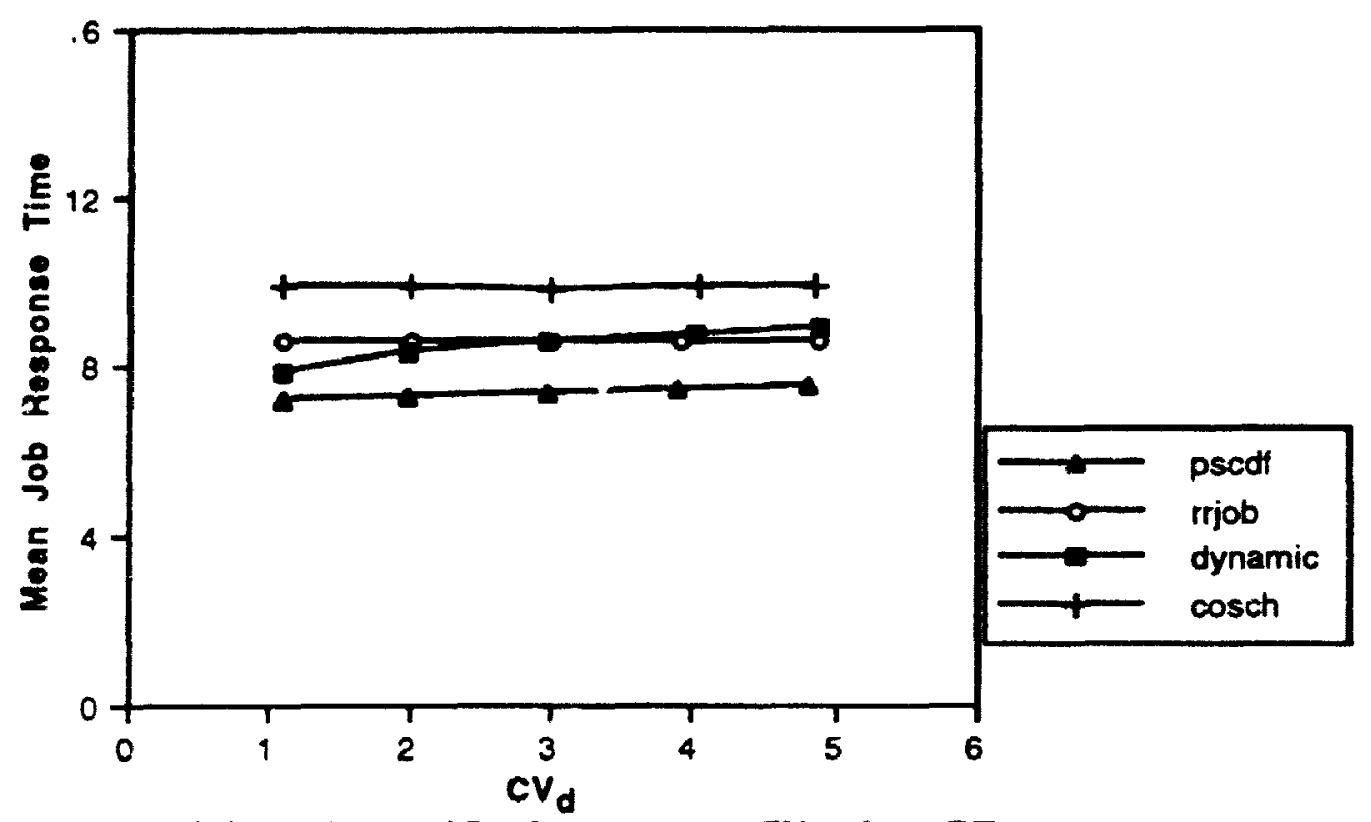

Figure 5.13 Sensitivity of Performance to $C V_{d}$ (Cor, GE)

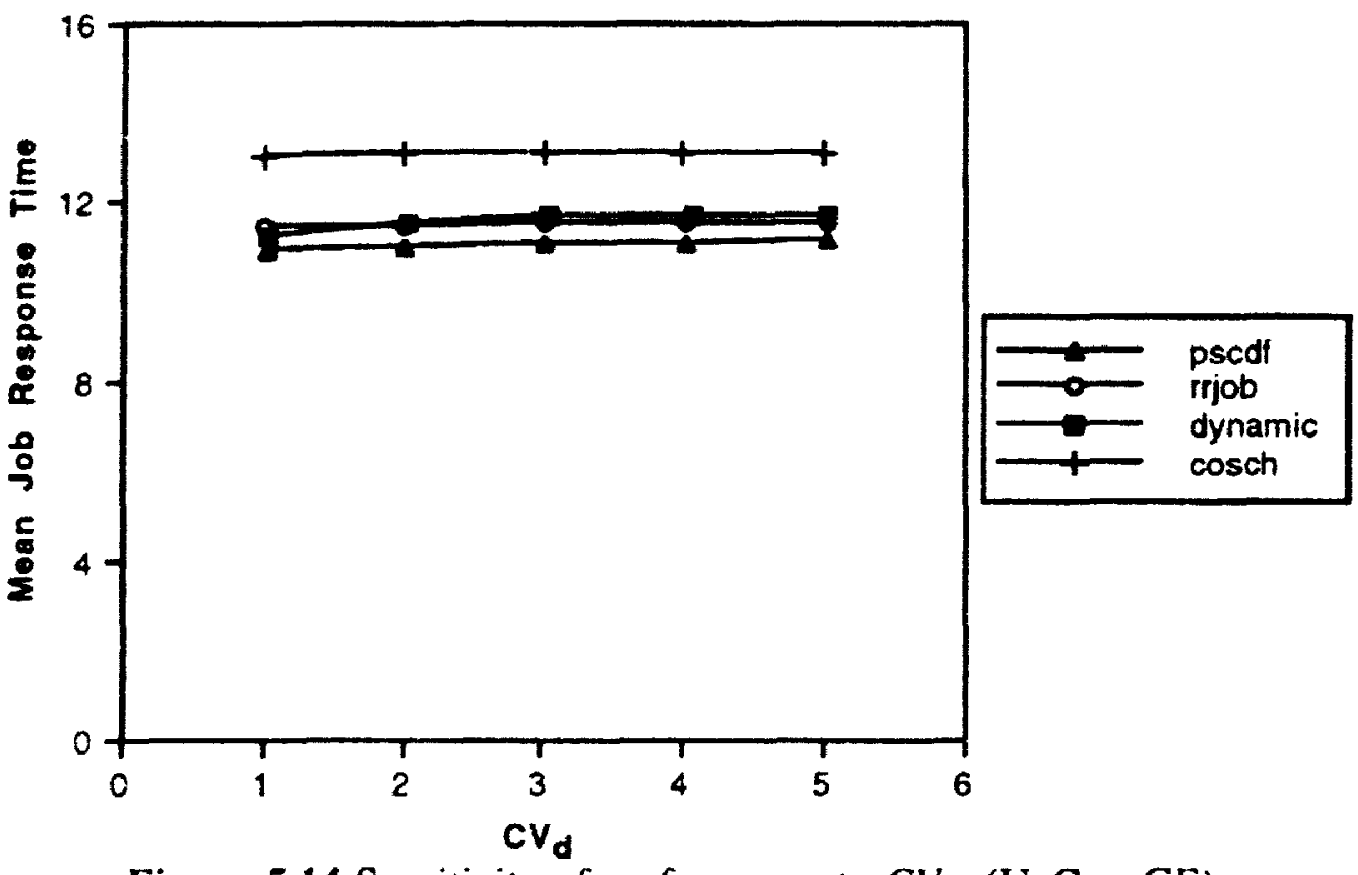

Figure 5.14 Sensitivity of performance to $C V_{d}$ (UnCor, GE) 


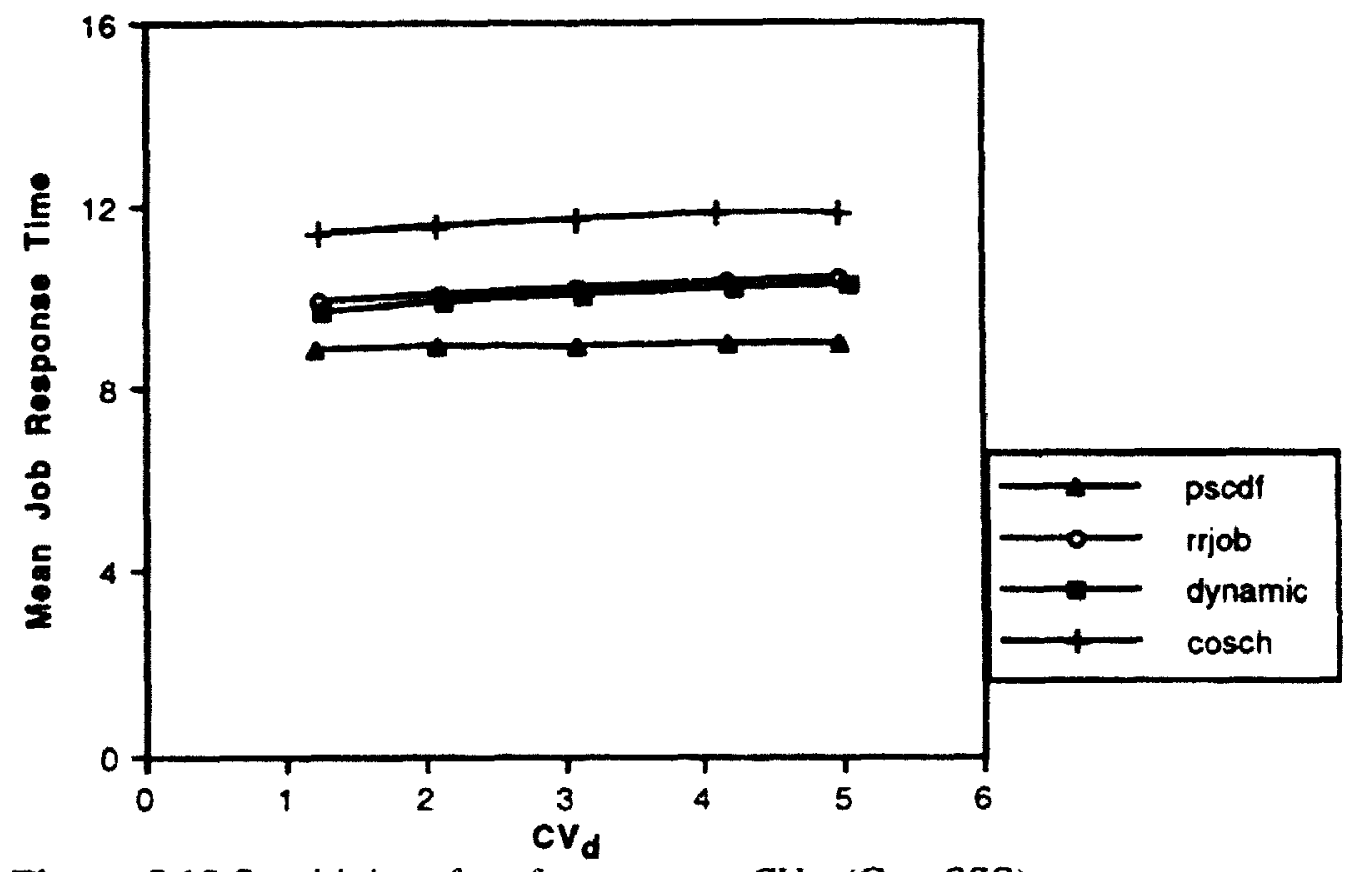

Figure 5.15 Sensitivity of performance to $C V_{d}$ (Cor, SSS)

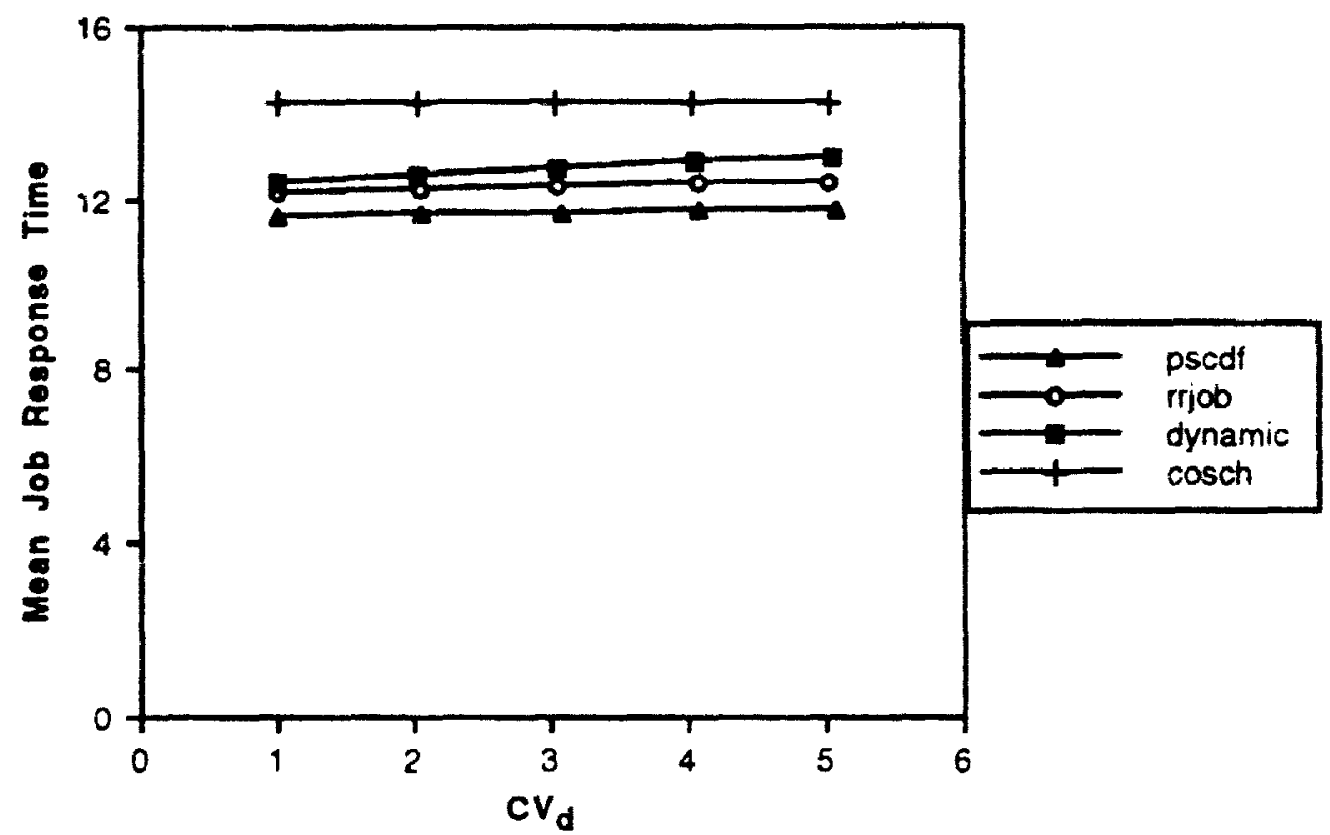

Figure 5.16 Sensitivity of Performance to $C V_{d}$ (UnCor, SSS) 


\subsection{Sensitivity of Performance to $n$}

In this section, we present the sensitivity analysis of $n$. We fix the system utilization level at 70\%. Other parameters are set as follows: $C V_{n}=1.0, d=20.0$ and $C V_{d}=5.0 \ldots$ dll the job models. We perform the experiments were conducted for values of $n=10.0 .15 .0$. 20.030 .0 and 40.0 for both the correlated and uncorrelated workload.

\subsubsection{Results for the Uncorrelated Workload}

The simulation results for the fork-and-join job model is shown in Figure 5.17. The default values of the input parameters for this experiment are given in Table 5.6.

\begin{tabular}{|c|c|c|c|c|}
\hline Input & \multicolumn{4}{|c|}{ Default Values } \\
Parameters & FJ & DC & GE & SSS \\
\hline$m p$ & vary & vary & vary & vary \\
\hline$C V_{m p}$ & 1.0 & 1.0 & 1.0 & 1.0 \\
\hline$d$ & 20.0 & 20.0 & 20.0 & 20.0 \\
\hline$C V_{d}$ & 5.0 & 5.0 & 5.0 & 5.0 \\
\hline$P_{T}$ & 0.0 & 0.0 & 0.0 & 0.5 \\
\hline
\end{tabular}

Table 5.6 Default values of the input parameters (uncorrelated workload)

(Sensitivity of performance to $\mathrm{n}$ ) 


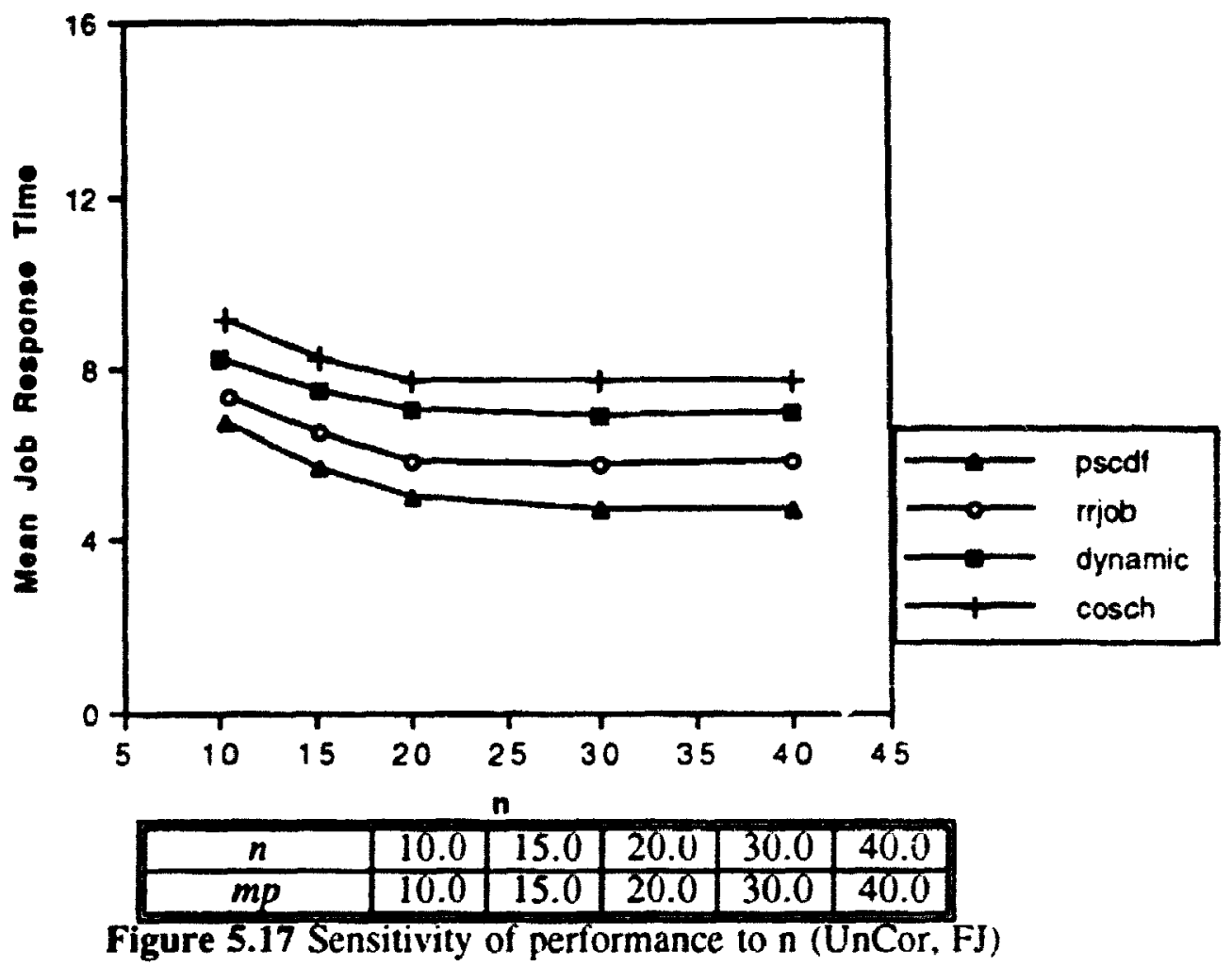

Similar experiments have also been conducted by [Leutenegger 90]. We extend the experiment to consider the cases where $n \geq 20$ (the number of processors in the system). The results of the uncorrelated workload is first presented in this subsection. As $n$ increases the mean job response time decreases up to $n=20.0$ and the mean job response time remains steady after that. An increase in $n$ introduces additional job parallelism and hence a decrease in the response time.

For the coscheduling policy, an increase in job parallelism results in a shorter execution time per job. However, when $n=20.0$ the advantage of additional parallelism is limited by the existing resources and, hence, no further performance improvement can the obtained. The analysis for the rrjob is similar to the coscheduling. For the dynamic policy. an increase in job parallelism improve the ability of each job to utilize the system's 
resources. However, the advantage of additional parallelism is limited hy the number of processors that can be allocated to each job.

Figure 5.18 plots $n$ versus the average job response time for the divide-and-conquer joh model.

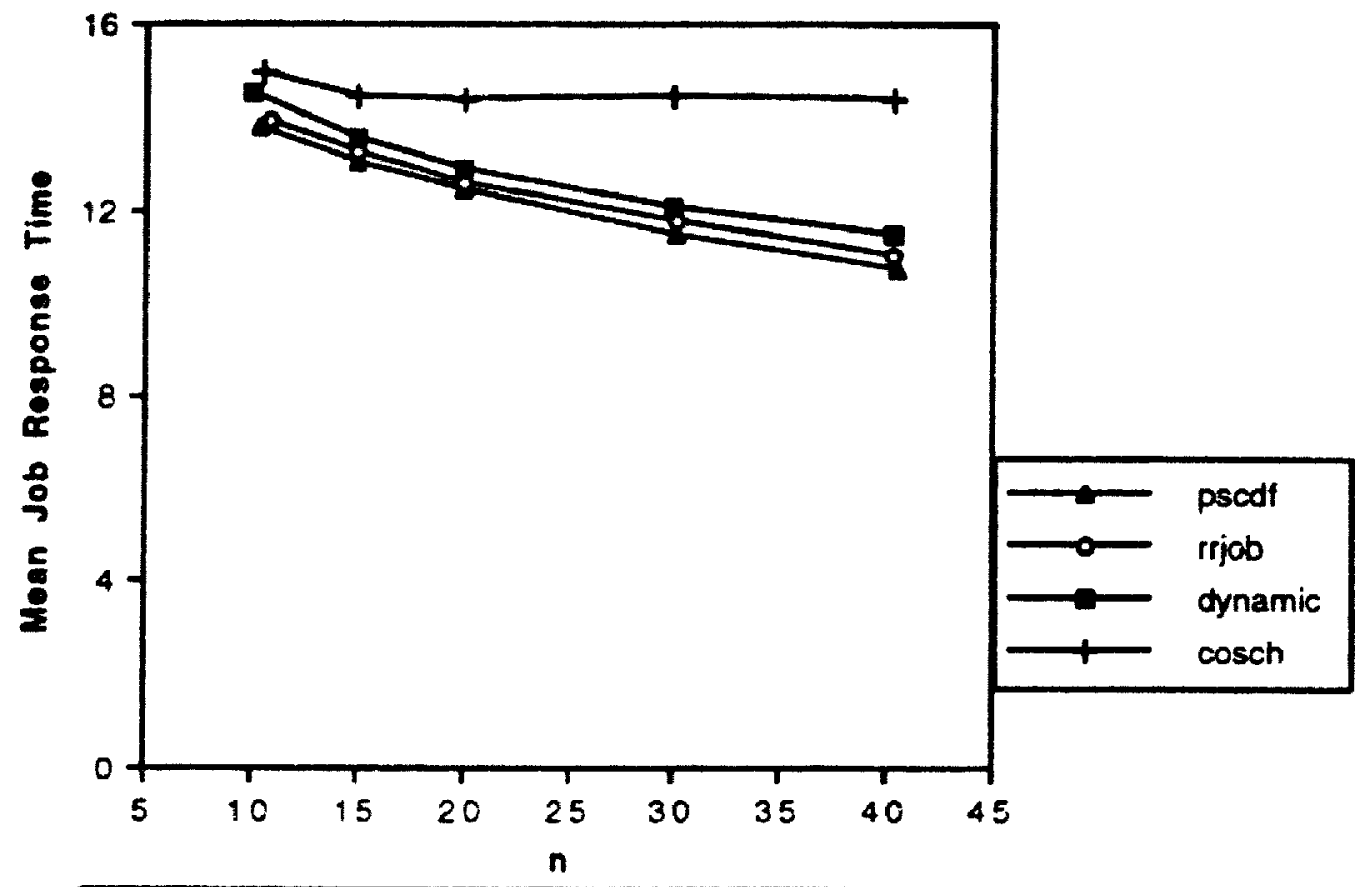

\begin{tabular}{|c|c|c|c|c|c|}
\hline $\mathrm{n}$ & 10.3627 & 16.0435 & 20.038 & 31.052 & 41.464 \\
\hline $\mathrm{mp}$ & 7.5 & 8.0 & 12.444 & 16.0 & 22.5 \\
\hline
\end{tabular}

Figure 5.18 Sensitivity of performance to $\mathrm{n}$ (UnCor, DC)

For the divide-and-conquer job model, within the range of $n$ values considered were, the average maximum parallelism is approximately 16.0 . When $n$ is 40.0 , the parallelism per jut is stili less than the number of processors. Thus, the advantage of additional parallelism is not affected hy the system's resources. For the coscheduling policy, the decrease in mean joh response time is only marginal. Thi, shows that coscheduling is not suitable for divide-and-comquer johs, when jobs have higher degree of parallelism. 
The resuits of the GE and SSS job models are shown in Figure 5.19 and 5.20. The results are is similar to one for the divide-and-conquer job model.

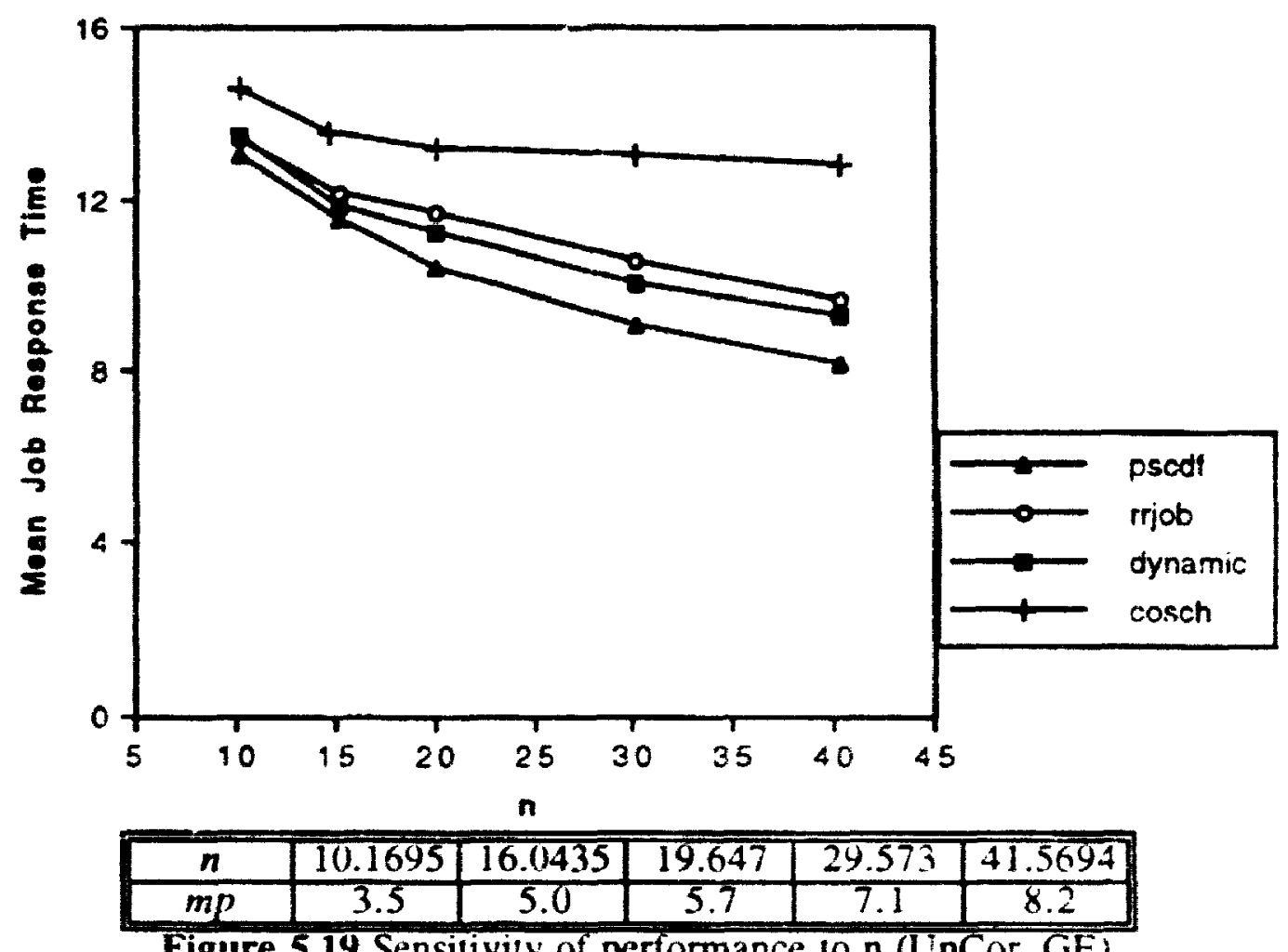

Figure 5.19 Sensitivity of performarce to $n$ (UnCor, GE) 

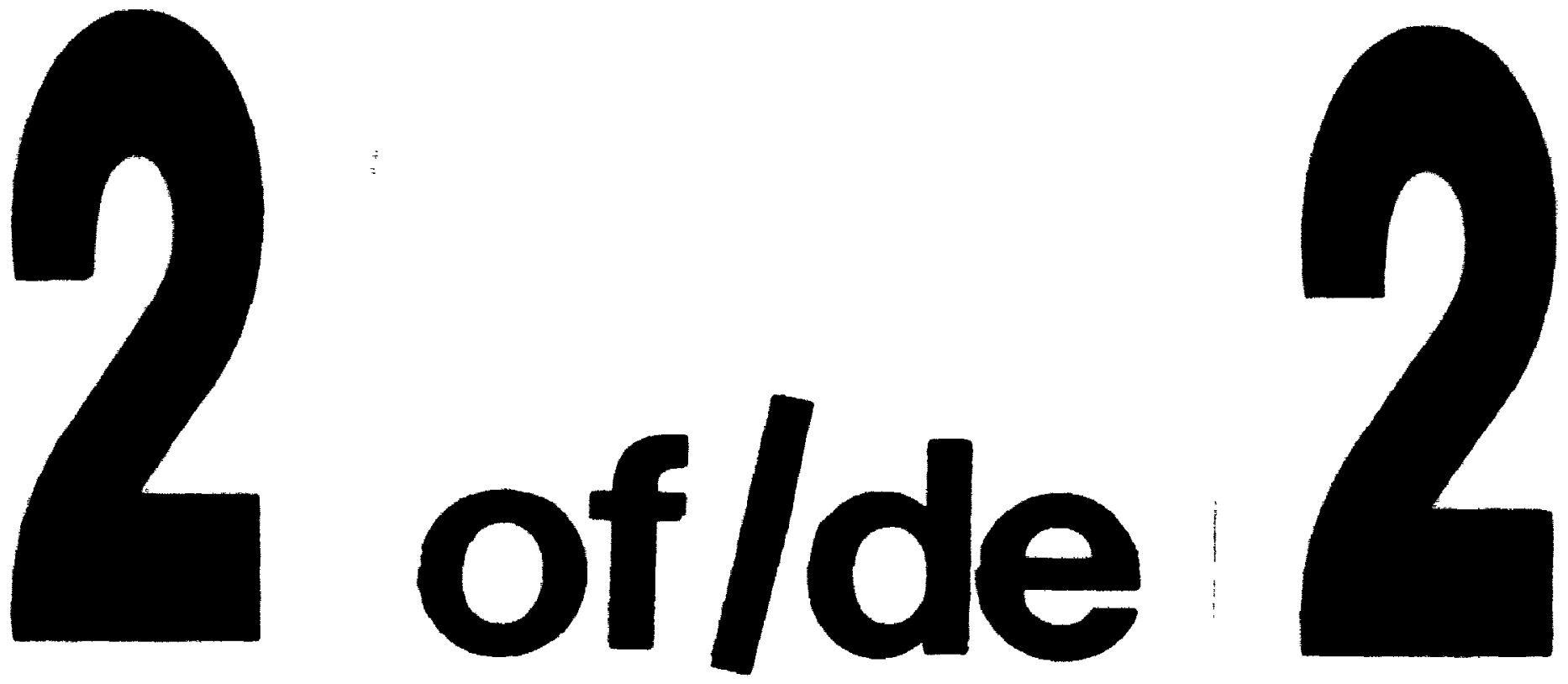

PM.1 3" ":4" PHOTOGRAPHIC MICROCOPY TARGET NAS 1010a ANSI/ISO \#2 EOUIVALENT

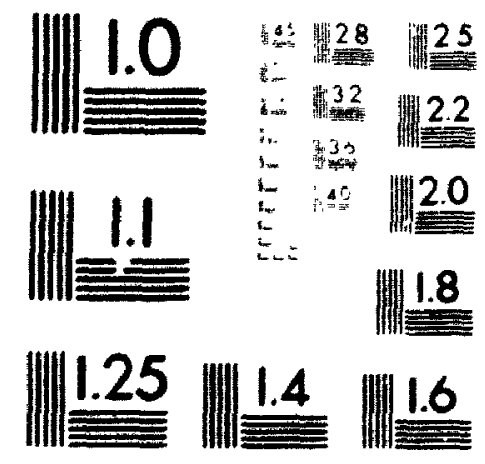

PRECISIONSM RESOLUTION TARGETS 


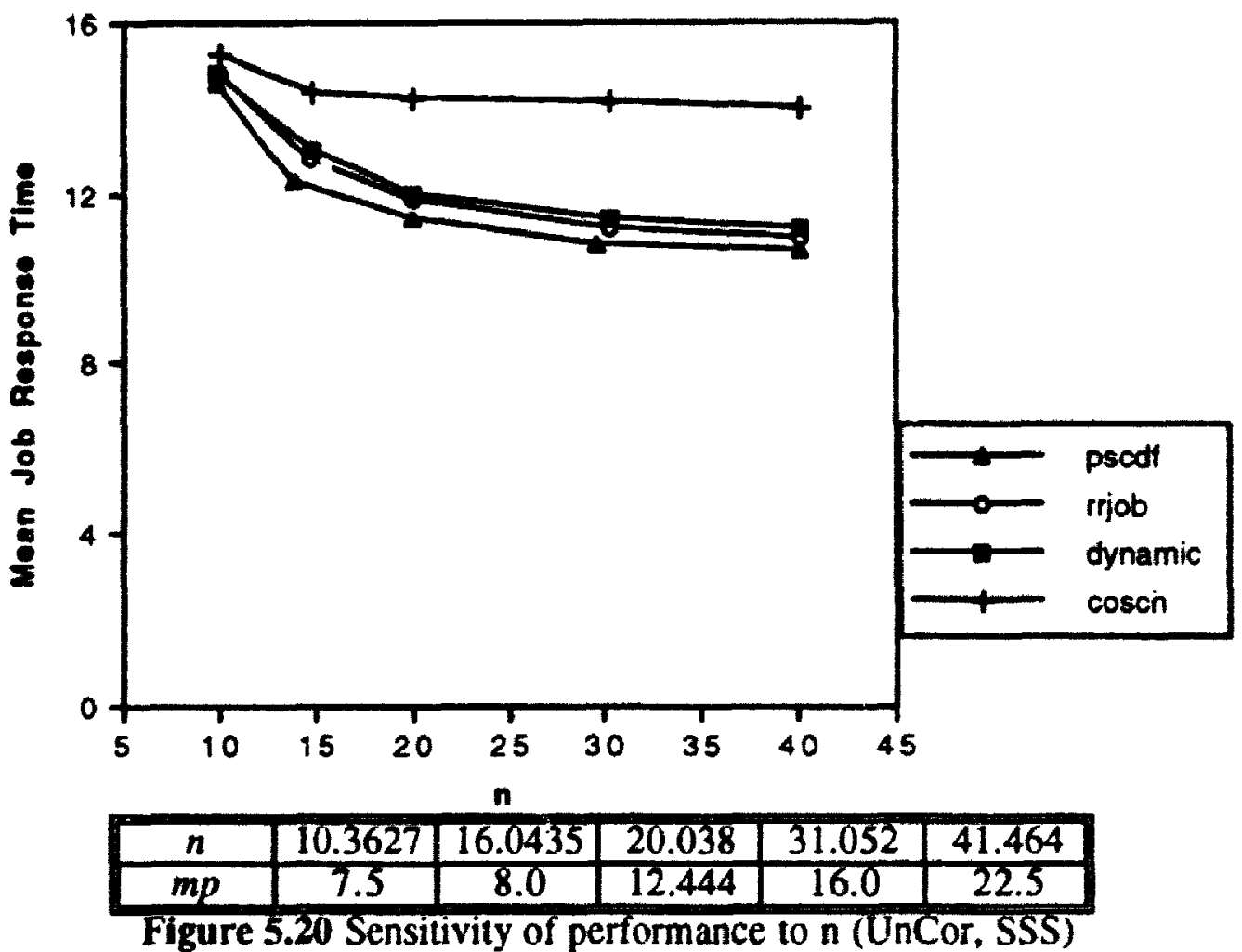

\subsubsection{Results of Correlated Workload}

The sensitivity analysis of $\boldsymbol{n}$ is also performed for correlated workload. The results are presented in Figure 5.21 through 5.24. Note that, in this workload, there is a positive correlation between the service demand and the number of tasks of a job. This is reasonable, since in real situations the service demand of individual tasks shoulc stay unaffected by the number of tasks. Consider the case of a program for Gaus sian elimination. Tasks are responsible for the computation of the dot-product of their associated rows and columns. Thus, an increase in the number of tasks corresponds to an increase in the size of the input matrices, which implies an increase in job service demand. A similar argument can also be made for the divide-and-conquer job model. In the experiment, $\mathrm{n}$ is set equal to 10.0,15.0,20.0,30.0 and 40.0. We vary the arrival rate so that the utilization 
level of the system is approximately equal to $70 \%$, for all values of $n$. The default values of the input parameters are given in Table 5.7.

\begin{tabular}{|c|c|c|c|c|}
\hline Input & \multicolumn{4}{|c|}{ Default Values } \\
Parameters & FJ & DC & GE & SSS \\
\hline$m p$ & vary & vary & vary & vary \\
\hline$C V_{m p}$ & 1.0 & 1.0 & 1.0 & 1.0 \\
\hline$d$ & 1.0 & 1.0 & 1.0 & 1.0 \\
\hline$C V_{d}$ & 5.0 & 5.0 & 5.0 & 5.0 \\
\hline$P_{T}$ & 0.0 & 0.0 & 0.0 & 0.5 \\
\hline
\end{tabular}

Table 5.7 Default values of the input parameters (correlated workload)

(Sensitivity of Performance to $n$ )

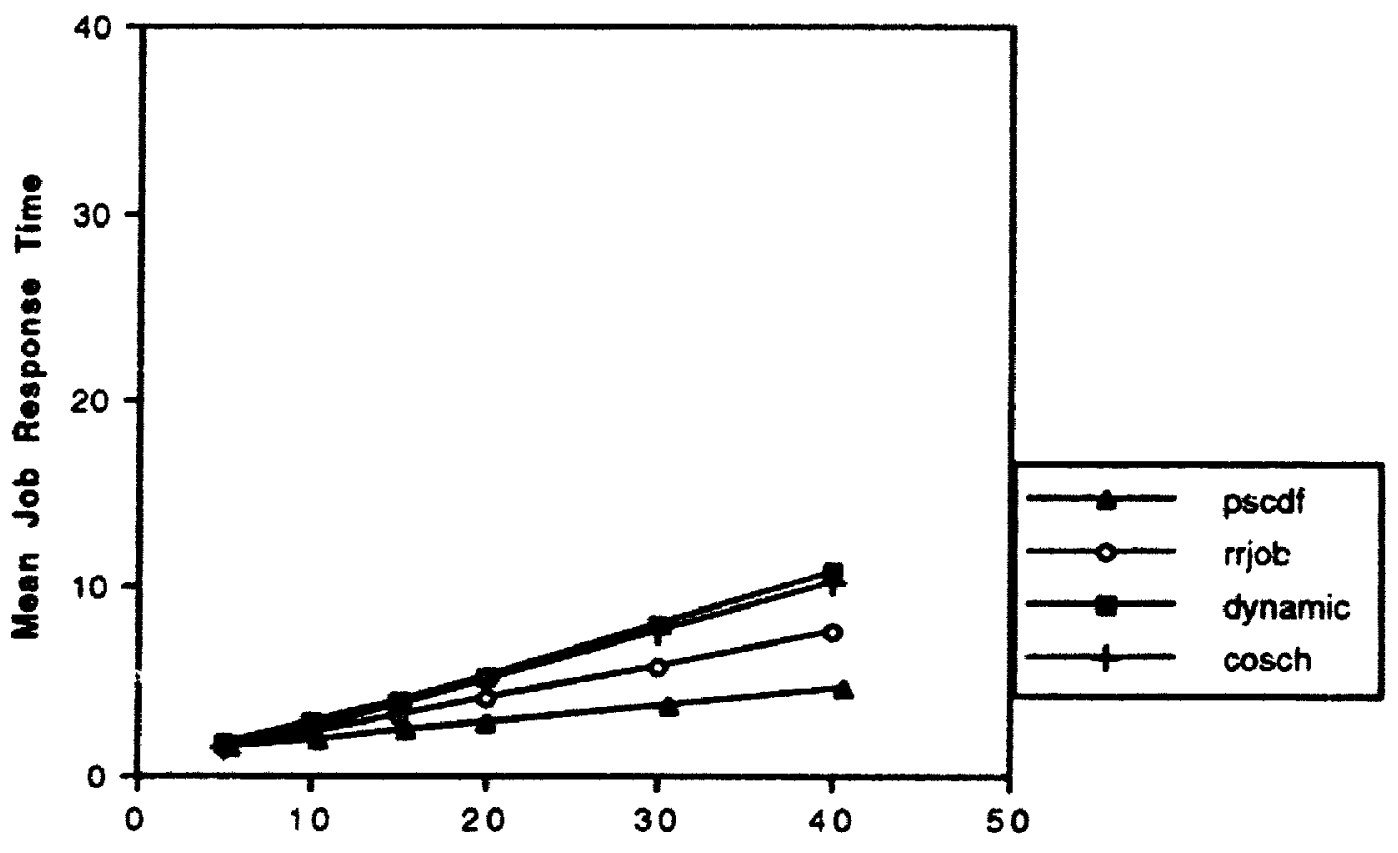

\begin{tabular}{|c|c|c|c|c|c|c|}
\hline$n$ & 5.0 & 10.0 & 15.0 & 20.0 & 30.0 & 40.0 \\
\hline$m p$ & 5.0 & 10.0 & 15.0 & 20.0 & 30.0 & 40.0 \\
\hline$d($ output) & 4.9843 & 9.9731 & 15.006 & 19.973 & 29.943 & 40.003 \\
\hline
\end{tabular}

Figure 5.21 Sensitivity of performance to $n(\mathrm{Cor}, \mathrm{FJ})$ 


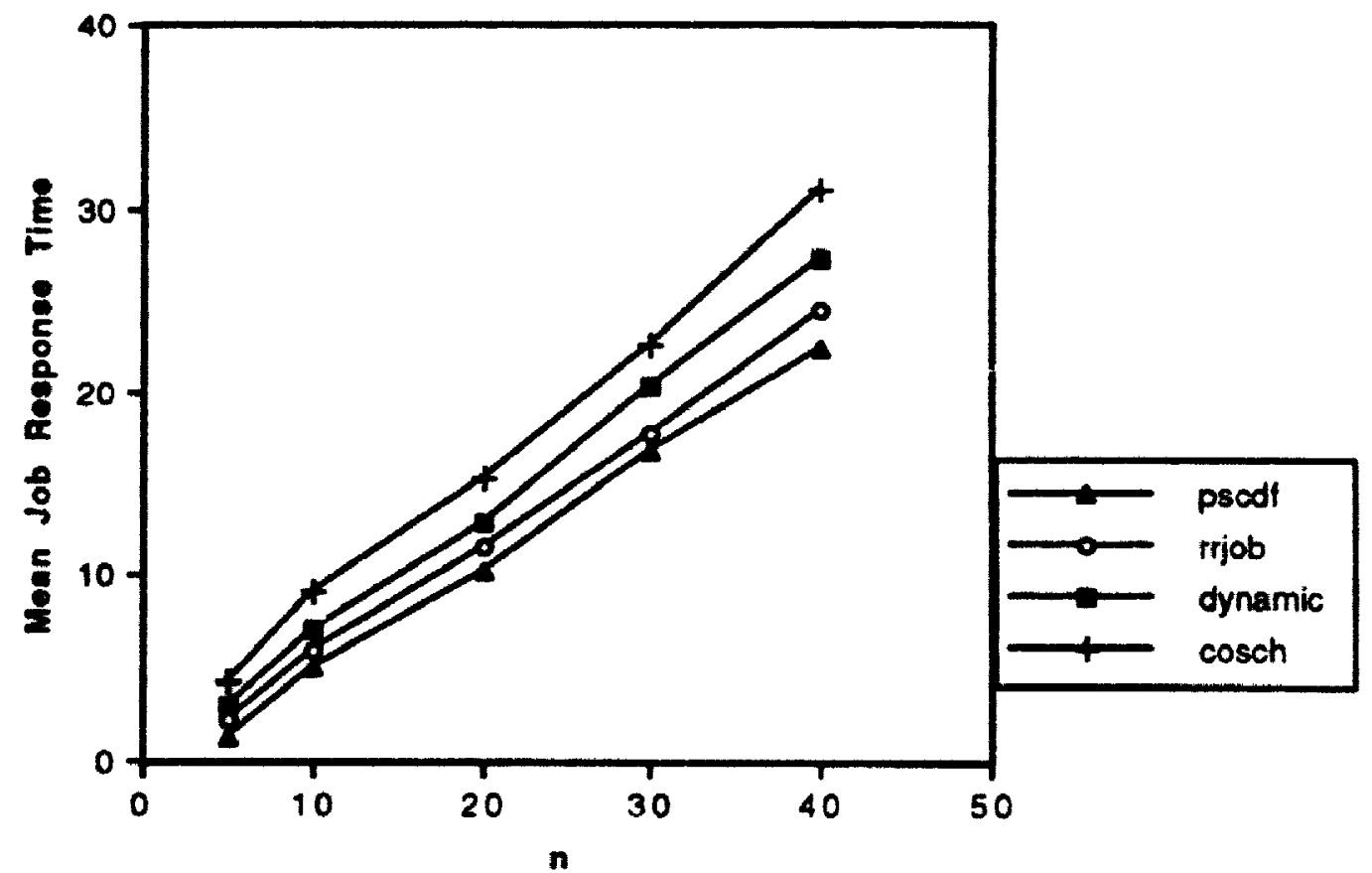

\begin{tabular}{|c|c|c|c|c|c|c|}
\hline$n$ & 5.034 & 10.3627 & 16.0435 & 20.038 & 31.052 & 41.464 \\
\hline$m p$ & 3.54 & 7.5 & 8.0 & 12.444 & 16.0 & 22.5 \\
\hline$d$ (output) & 5.130 & 10.127 & 16.5638 & 20.34 & 30.993 & 40.939 \\
\hline
\end{tabular}

Figure 5.22 Sensitivity performance to $\mathrm{n}$ (Cor, DC) 


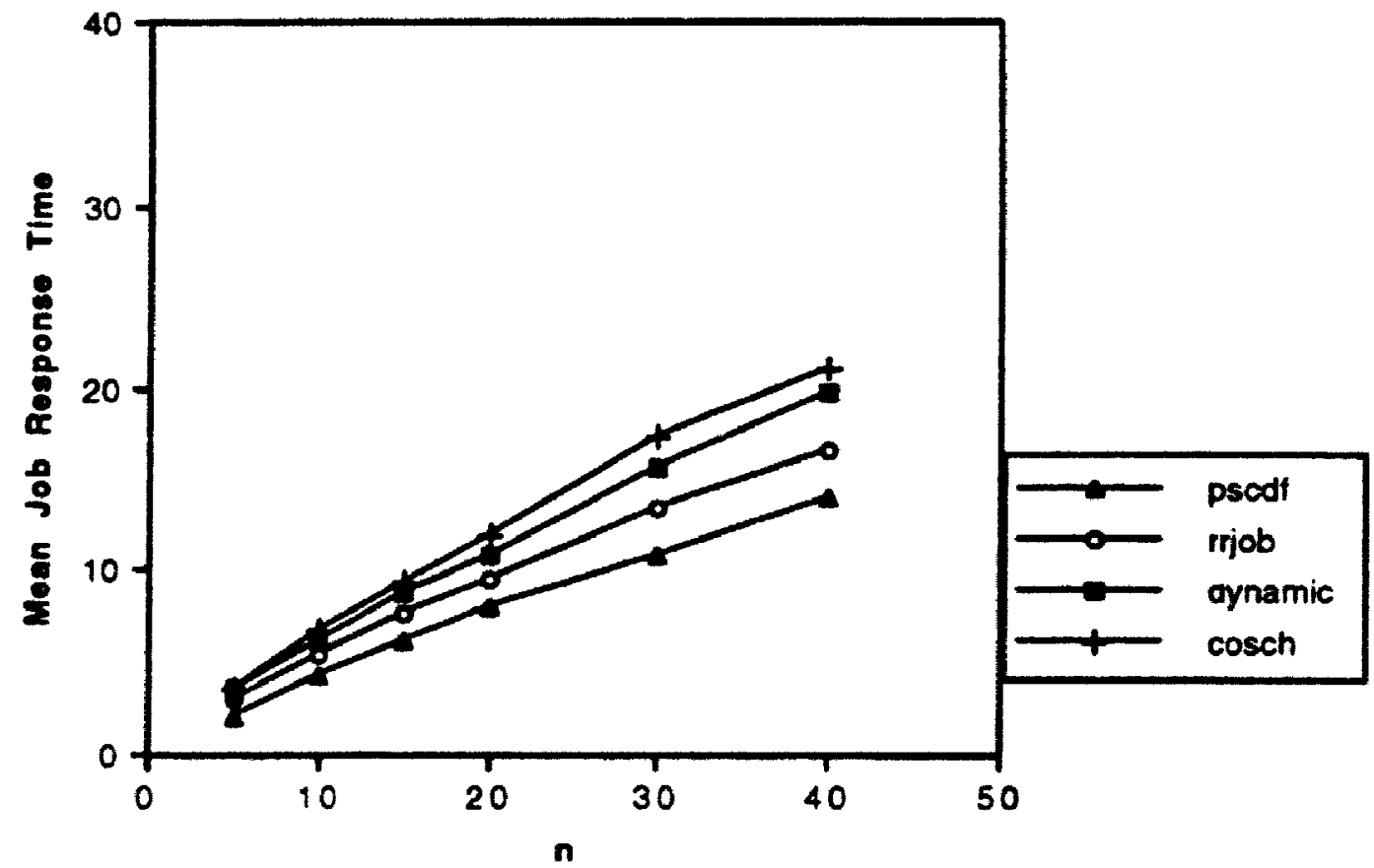

\begin{tabular}{|c|c|c|c|c|c|c|}
\hline$n$ & 5.054 & 10.1695 & 16.0435 & 19.647 & 29.573 & 41.5694 \\
\hline$m p$ & 2.87 & 3.5 & 5.0 & 5.7 & 7.1 & 8.2 \\
\hline$d($ output) & 5.204 & 10.1434 & 16.1133 & 19.754 & 29.773 & 41.3923 \\
\hline
\end{tabular}

Figure 5.23 Sensitivity of performance to $n$ (Cor, GE) 


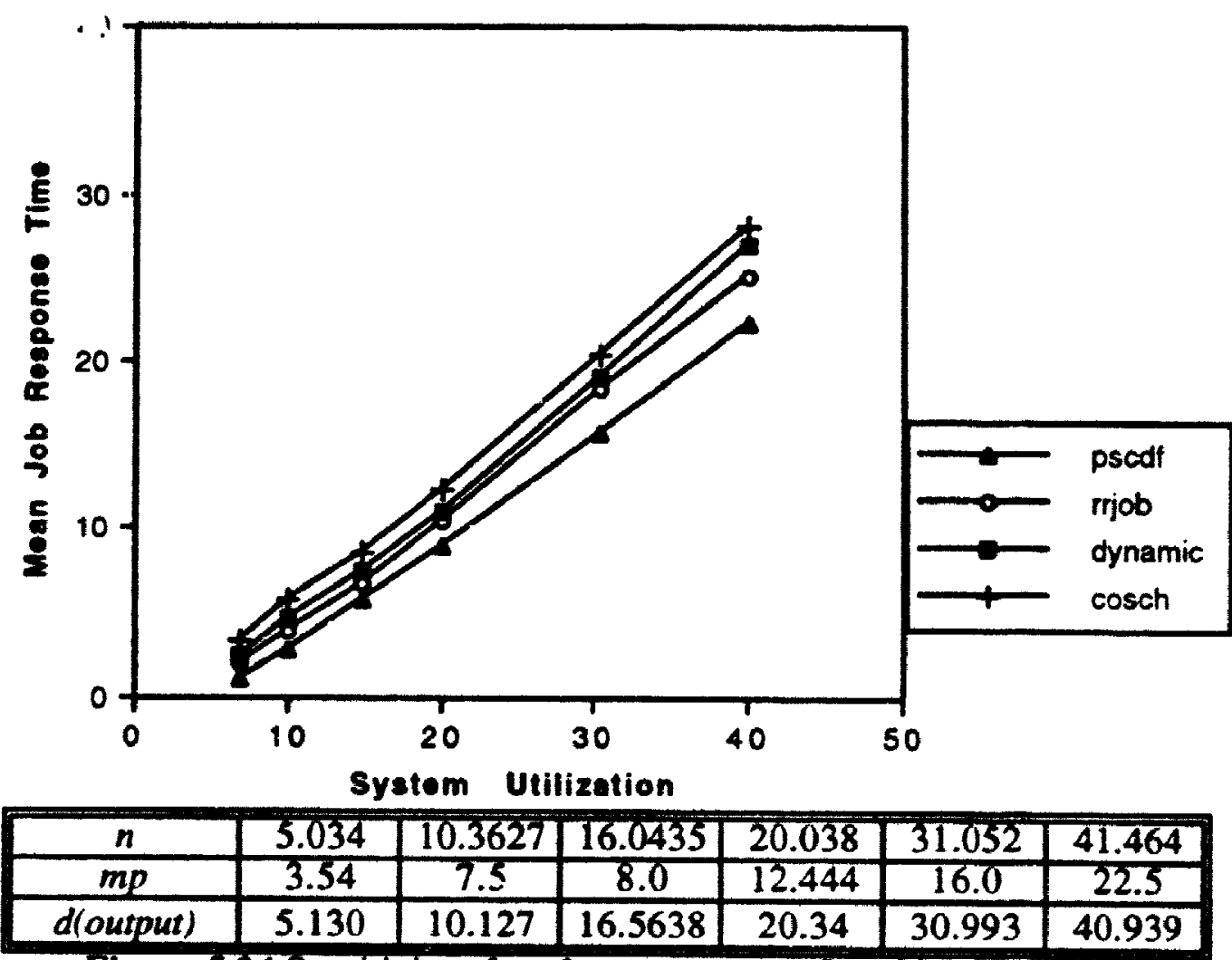

Figure 5.24 Sensitivity of performance to $n$ (Cor, SSS)

Due to the correlation between the job service demand and number of tasks, the mean job response time increases as we increase the values of $n$. Furthermore, there is no change in the relative performance among the policies, as we increase the number of tasks.

\subsection{Impact of $C V_{n}$ on The Performance of Scheduling Policies}

Experiments for different levels of utilization were performed with $C V_{n}=0.0$ and 5.0. $C V_{n}=0.0$ represents the case where all jobs have the same number of tasks. $C V_{n}=5.0$ represents the situation where the workload consists of large number of jobs with sinall number of tasks and small number of jobs with a large number of tasks. The input parameters for this experiment are shown in Tables 5.8 and 5.9. 


\begin{tabular}{|c|c|c|c|c|}
\hline Input & \multicolumn{4}{|c|}{ Default Values } \\
Parameters & FJ & DC & GE & SSS \\
\hline$m p$ & 20.0 & 12.444 & 5.667 & 12.444 \\
\hline$C V_{m p}$ & 0.0 or 5.0 & 0.0 or 5.0 & 0.0 or 5.0 & 0.0 or 5.0 \\
\hline$d$ & 20.0 & 20.0 & 20.0 & 20.0 \\
\hline$C V_{d}$ & 5.0 & 5.0 & 5.0 & 5.0 \\
\hline$P_{T}$ & 0.0 & 0.0 & 0.0 & 0.5 \\
\hline
\end{tabular}

Table 5.8 Default values of the input paraneters (uncorrelated workload)

(Impact of $C V_{n}$ on The Performance of The Scheduling Policies)

\begin{tabular}{|c|c|c|c|c|}
\hline Input & \multicolumn{4}{|c|}{ Default Values } \\
Parameters & FJ & DC & GE & SSS \\
\hline$m p$ & 20.0 & 12.444 & 5.667 & 12.444 \\
\hline$C V_{m p}$ & 0.0 or 5.0 & 0.0 or 5.0 & 0.0 or 5.0 & 0.0 or 5.0 \\
\hline$d$ & 1.0 & 1.0 & 1.0 & 1.0 \\
\hline$C V_{d}$ & 5.0 & 5.0 & 5.0 & 5.0 \\
\hline$P_{T}$ & 0.0 & 0.0 & 0.0 & 0.5 \\
\hline
\end{tabular}

Table 5.9 Default values of the input parameters (uncorrelated workload)

(impact of $C V_{n}$ on The Performance of The Scheduling Policies) 


\subsubsection{Discussion of Results for $C V_{n}=0.0$}

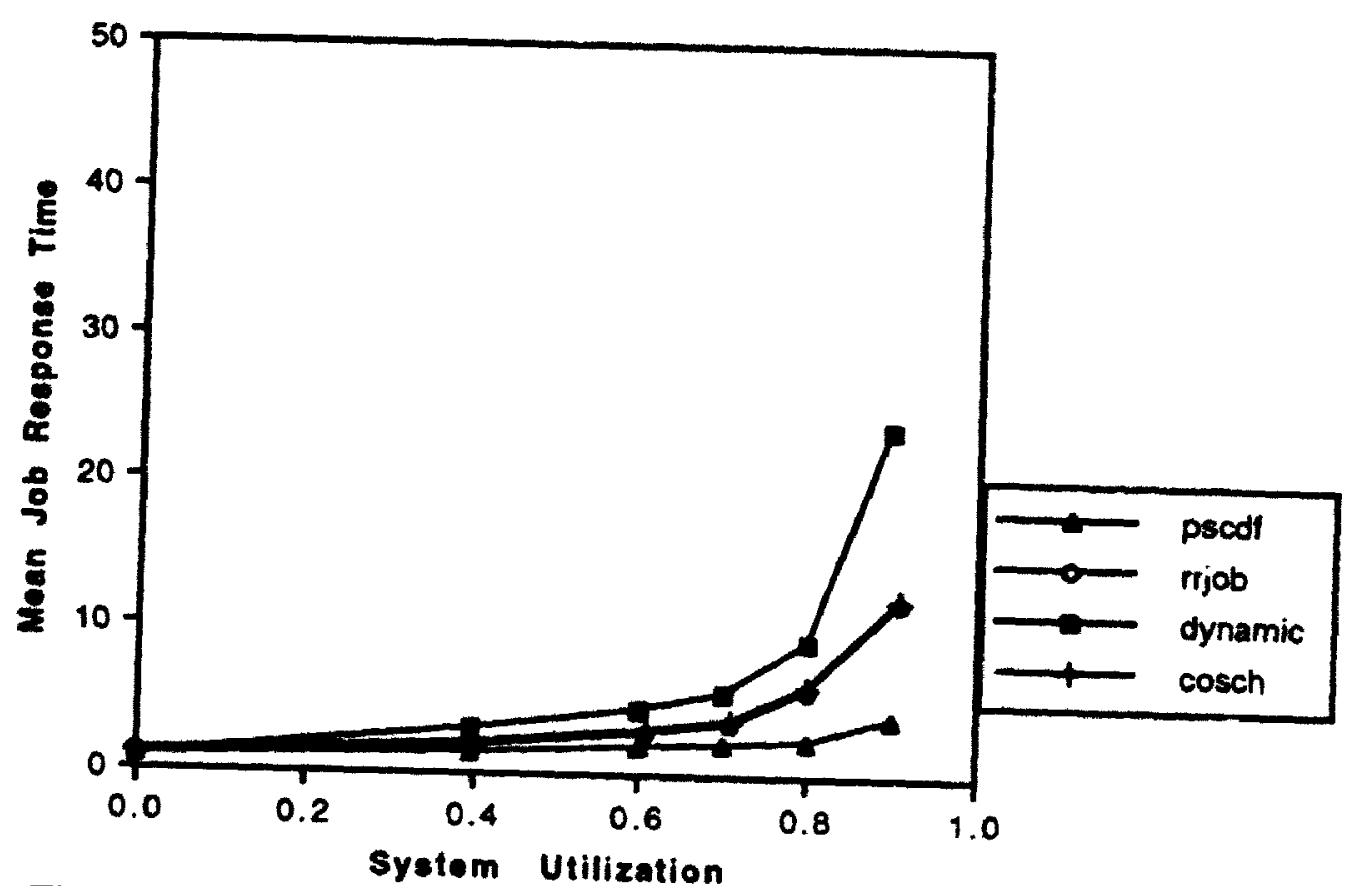

Figure 5.25 Performance of scheduling policies when $C V_{n}=0.0(\mathrm{Cor}, \mathrm{FJ})$

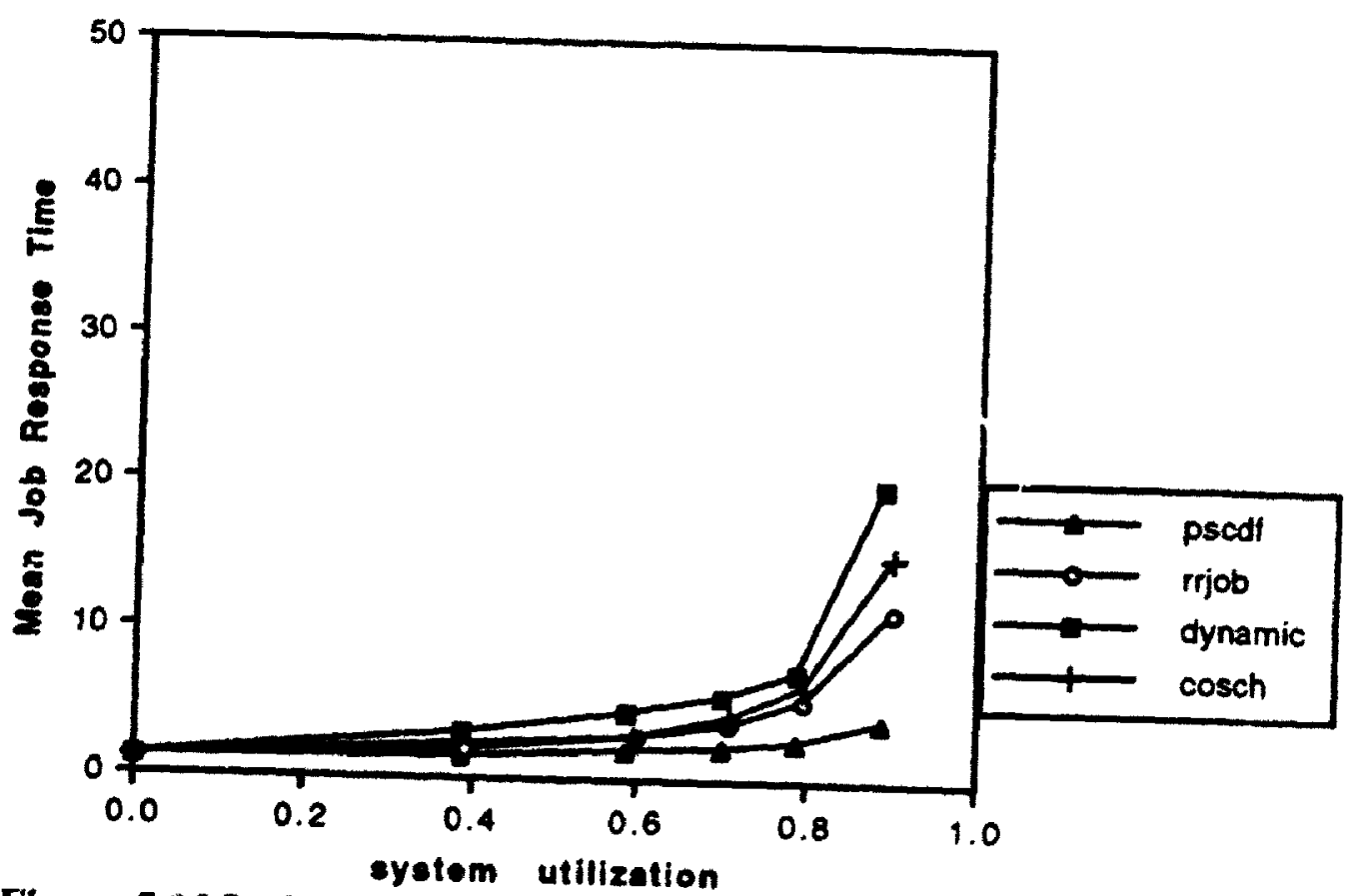

Figure 5.26 Performance of scheduling policies when $C V_{n}=0.0$ (UnCor,FJ) 
Figures 5.25 and 5.26 present the results for the correlated and uncorrelated versions of the fork-and-join job models with $C V_{n}=0.0$. Since the correlation between the service demand and the number of tasks breaks down as a result of using a constant number of tasks per job (because $C V_{n}=0.0$ ), the results for the uncorrelated workload is similar to those for the correlated workload. This can be seen by comparing Figures 5.25 and 5.26.

In a general setting, coscheduling tends to give large jobs consecutive quanta and hence increases the waiting time for the small jobs. Such a problem does not exist when the number of tasks per job is fixed and the performance of the coscheduling gets closer to the rjob. Under these circumstances, the rrjob maintains the same quantum size for each job. Thus, the scheduling sequence of this policy is similar to that in the coscheduling and, therefore, results in a similar performance.

Fixing the number of tasks per job does not yield performance benefits by using to the dynamic policy, as can be seen from the above results. At low to moderate system loads, the jobs with large service demand acquire more processors, as they stay in the system for a longer time. Small jobs that come after the large jobs will be allocated fewer processors than the large jobs. Such an unfair allocation of processors results in increased response time for the small jobs. 


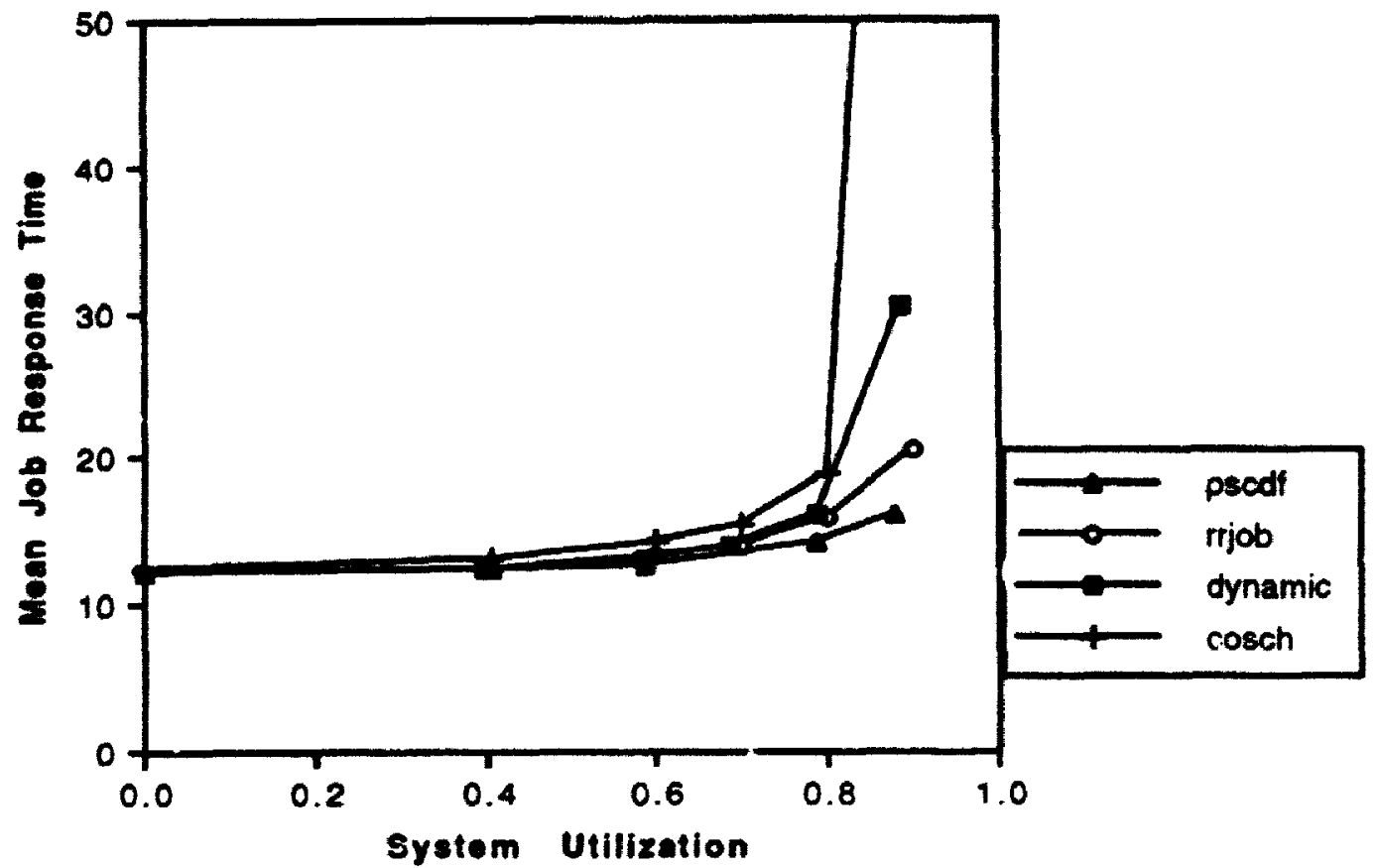

Figure 5.27 Performance of scheduling policies when $C V_{n}=0.0$ (Cor,DC)

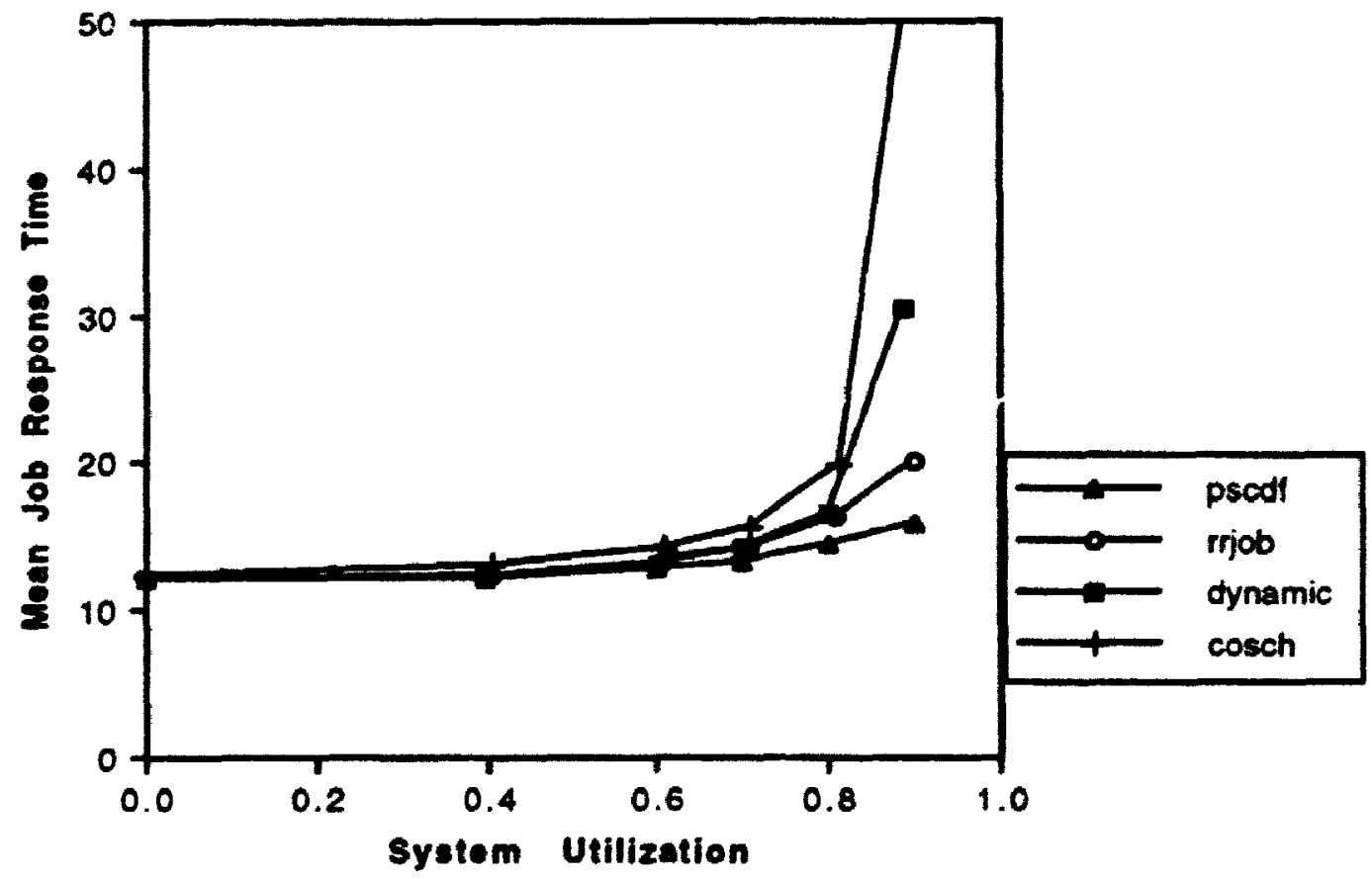

Figure 5.28 Performance of scheduling policies when $\mathrm{CV}_{n}=0.0$ (UnCor,DC)

Figures 5.27 and 5.28 present the results for the correlated and uncorrelated versions of the divide-and-conquer job model. Due to break down of the correlation between the 
service demand and number of tasks of the parallel jobs, the results for the two versions of the workload are similar.

Under the divide-and-conquer workload, the coscheduling policy gives the worst performance among the four policies. It performs worse than the rrjob mainly due to a larger synchronization delay induced by it. Although coscheduling is designed to reduce the synctironization delay of parallel jobs, it fails to do so under the divide-and-conquer workload. The group of tasks created from different branches of a job may be scheduled in different quanta, as each group is placed at different places in the queue. As a result of this, the synchronization delay of the jobs increases. On the other hand, rrjob is implemented in such a way that the tasks of a job is placed together in the job quelle, this results in minimizing synchronization delay of a job. In addition, the large contsxt switch overhead associated with the coscheduling policy also affects its performance.

Coscheduling performs worse than the dynamic mainly because it incurs large amount of context switch overhead. On the other hand, dynamic performs preemption only when a new job arrives or a job changes its parallelism. Therefore, the amount of overhead will be much less than that in the coscheduling policy.

Dynamic has a smaller context switch overhead by minimizing the number of context switches per job. However, it yields a longer synchronization delay per job. On the other hand, although the rrjob gives a shorter synchronization delay per job, its context switch overhead is larger than that of the dynamic policy. These two policies are comparable up to the utilization level of $80 \%$, then the performance of the dynamic degrades as the number of jobs exceeds the number of processors. 


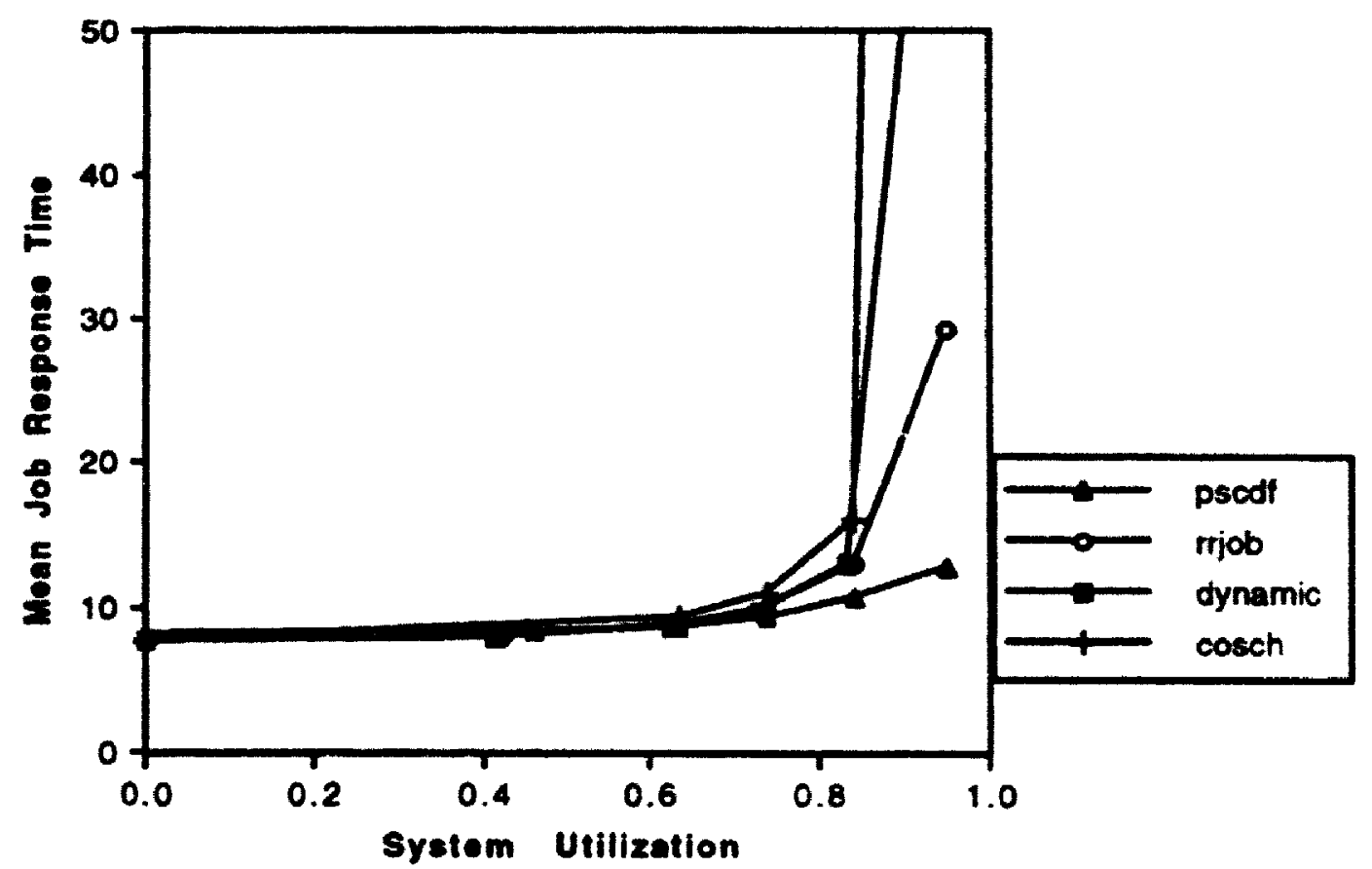

Figure 5.29 Performance of scheduling policies when $C V_{n}=0.0$ (Cor,GE)

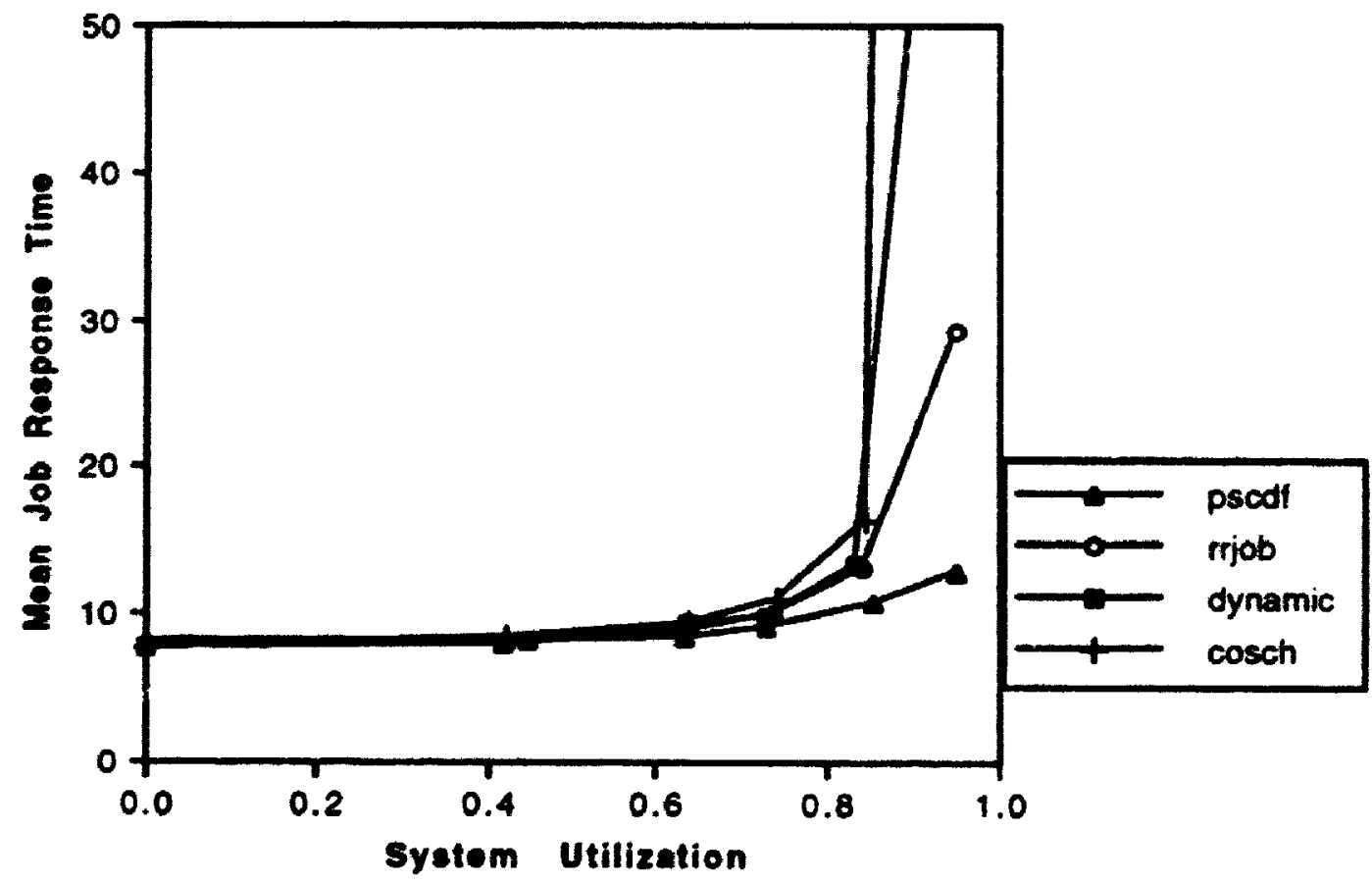

Figure 5.30 Performance of scheduling policies when $C V_{n}=0.0$ (UirCor, GE) 
Figures 5.29 and 5.30 present the results for the correlated and uncorrelated version of the workload for the gaussian elimination job model. The reason for the similar results for these two versions of the workload was discussed previously.

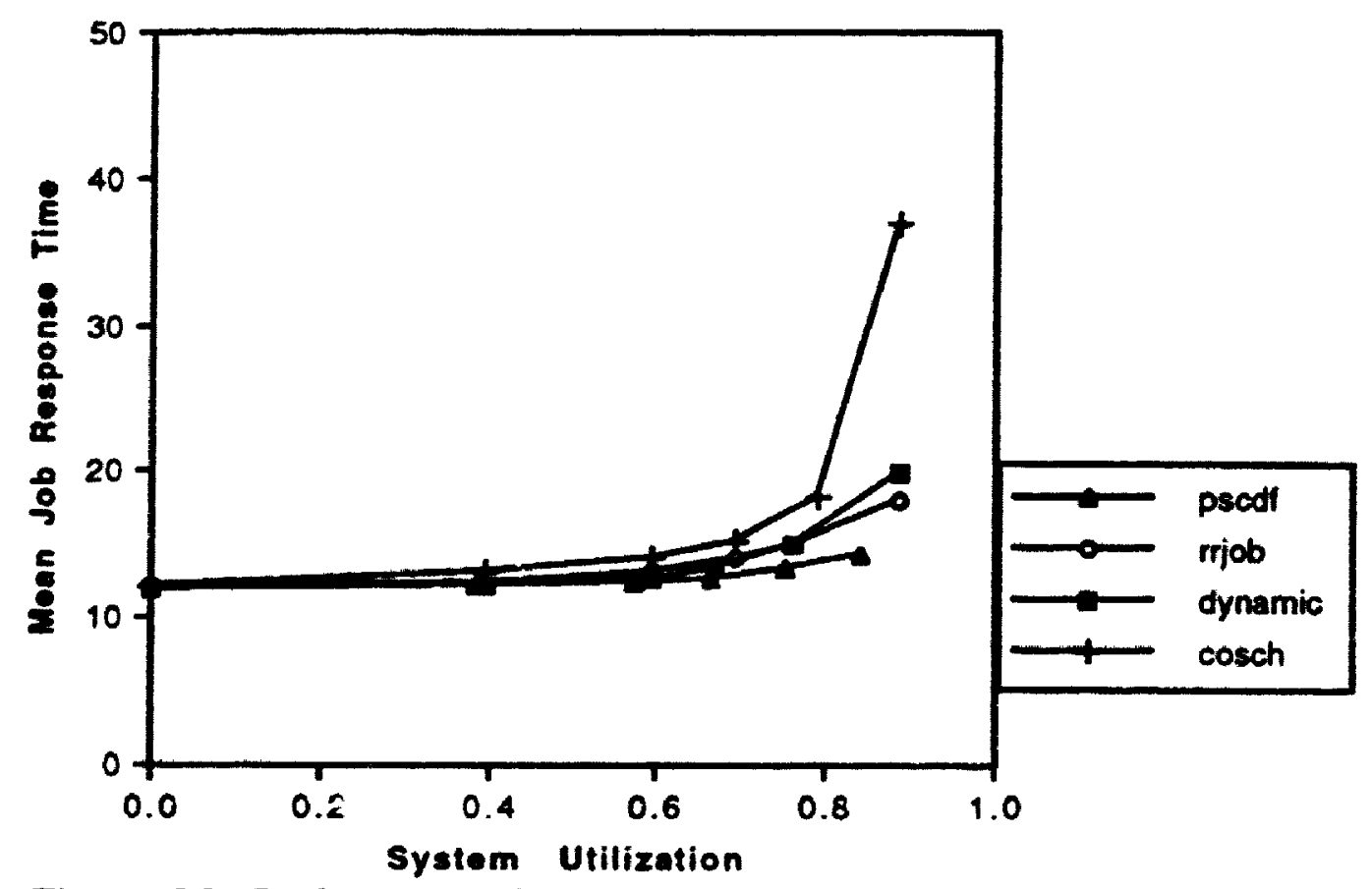

Figure 5.31 Performance of scheduling policies when $C V_{n}=0.0$ (Cor,SSS) 


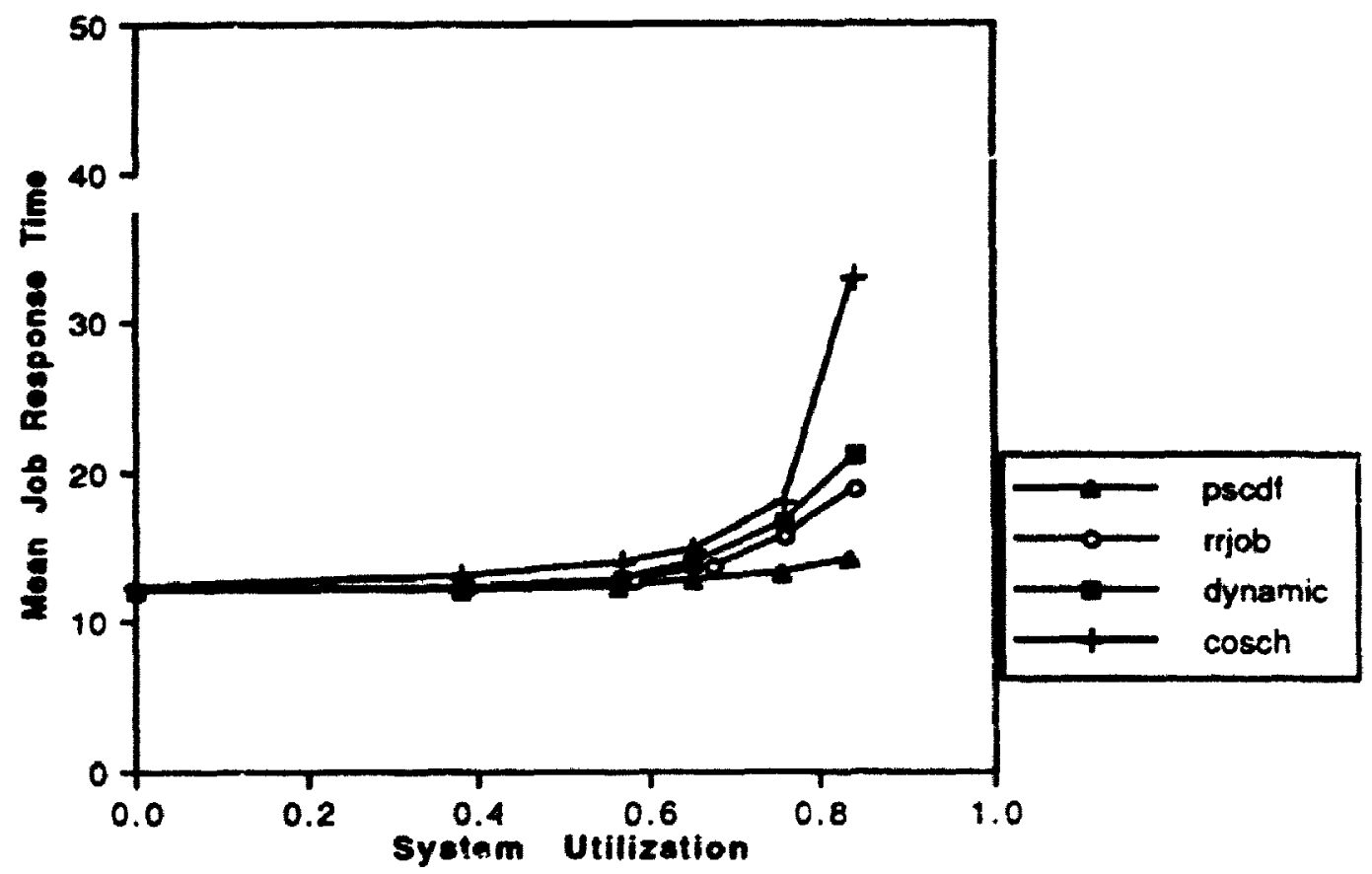

Figure 5.32 Performance of scheduling policies when $C V_{n}=0.0$ (UnCor,SSS)

The relative performance of the four policies under the state-space search job model is similar to that of the divide-and-conquer job model. As mentioned previously, the presence of task abortion does not change the relative performance. The reasons for the poor performance of coscheduling and the similar performance between rrjob and dynamic follow from the discussion of the divide-and-conquer job model.

\subsubsection{Discussion of Results for $C V_{n}=5.0$}

The results of the correlated and uncorrelated versions of the fork-and-join workload model with $C V_{n}=5.0$ are presented in Figure 5.33 and 5.34 . 


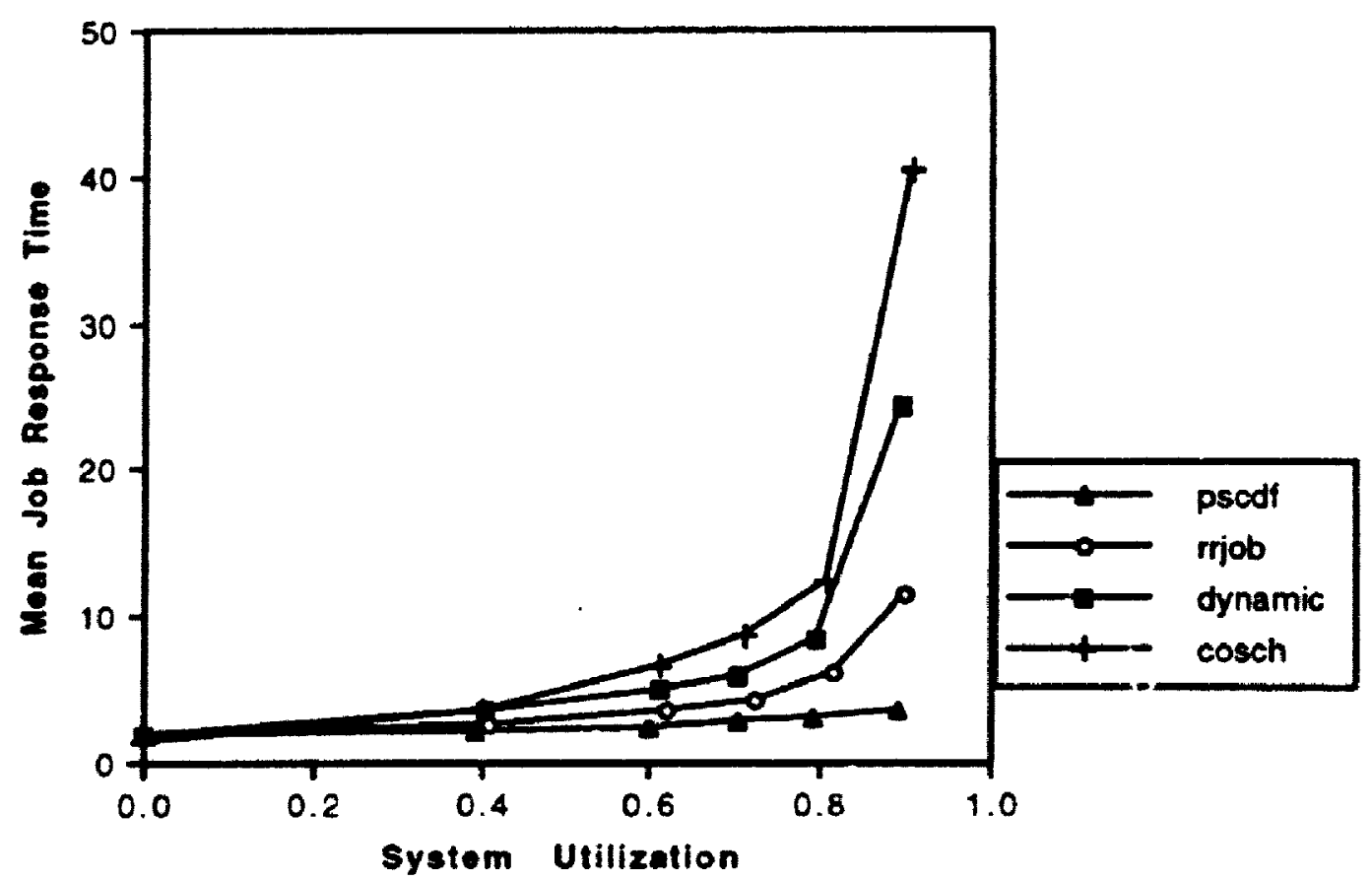

Figure 5.33 Performance of scheduling policies when $C V_{n}=5.0$ (Cor,FJ)

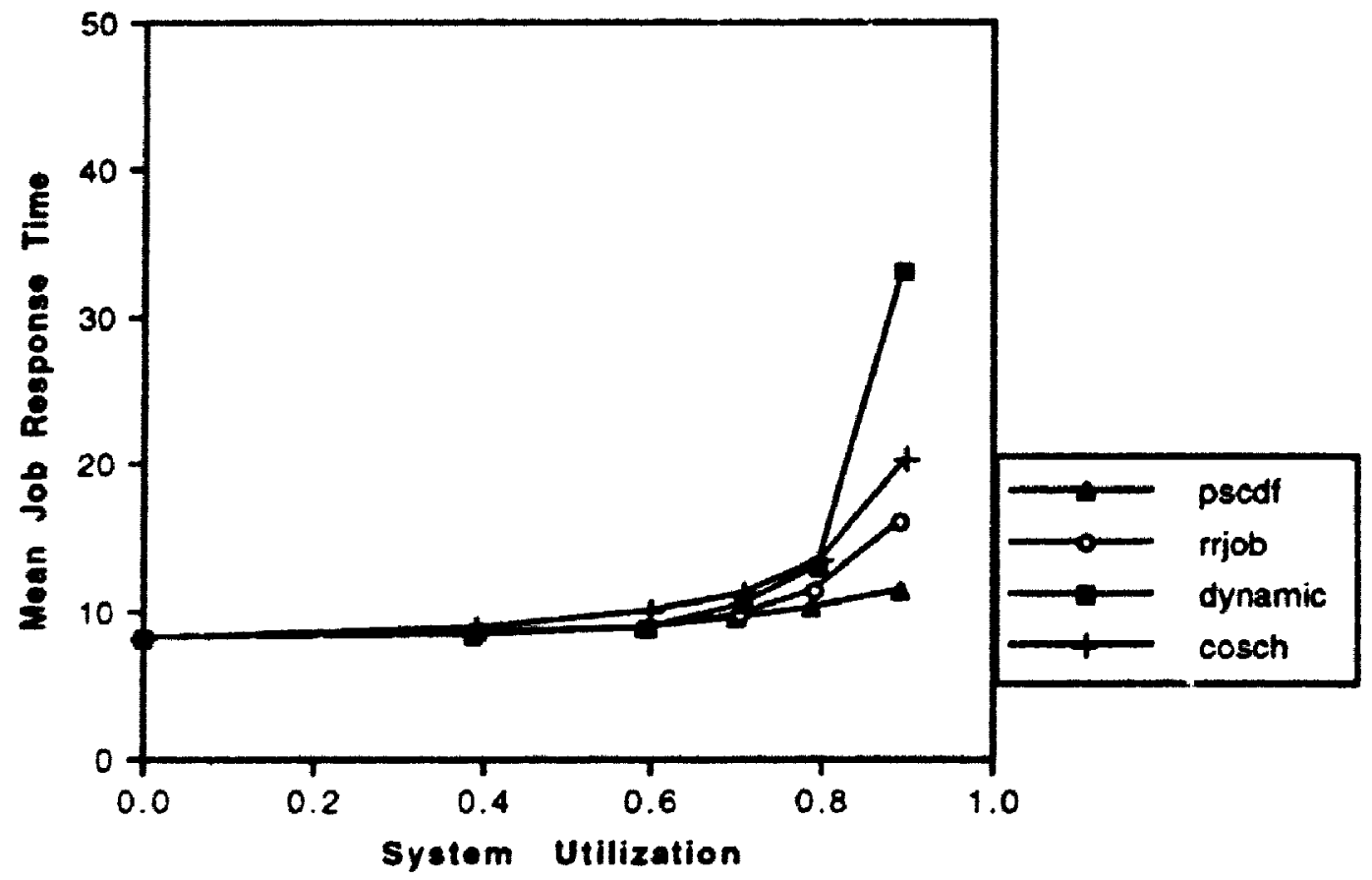

Figure 5.34 Performance of scheduling policies when $C V_{n}=5.0$ (UnCor,FJ) 
The performance of the policies under the correlated workload is discussed first. Under this workload, there are $20.561 \%$ of sequential jobs, $66.159 \%$ of jobs with 1 to 10 tasks, $7.4066 \%$ of jobs with 10 to 20 tasks and $5.381 \%$ of jobs with more than 20 tasks.

When the workload is correlated, jobs with large number of tasks tend to have larger service demand. Thus, coscheduling performs poorly, as a result of providing disproportionate share of the system. As discussed in the previous section, the dynamic policy gives an unequal share of the processing power among the jobs. This causes the policy to perform worse than the rrjob.

On the other hand, under the uncorrelated workload, the performance of the coscheduling is closer to that of the rijob policy. When $C V_{n}=5.0$, there are large number of jobs that are either sequential or have small amount of parallelism. Ioreover, in this case, there is no correlation between the service demand and number of tasks, so the jobs with large number of tasks may not have large service demand. Hence, even though the jobs with large number of tasks may take up series of quanta, their effects will be very limited. The dynamic policy performs poorly at moderate to high utilization levels. Since dynamic only allows as many jobs into the system as there are processors, the remaining jobs are kept in the queue until a job finishes execution. At moderate to high loads, the number of jobs in the system often exceeds the number of processors. As a result, the dynamic poiicy behaves similar to the first-come-first-served discipline and provides performance that is worse than the coscheduling. 


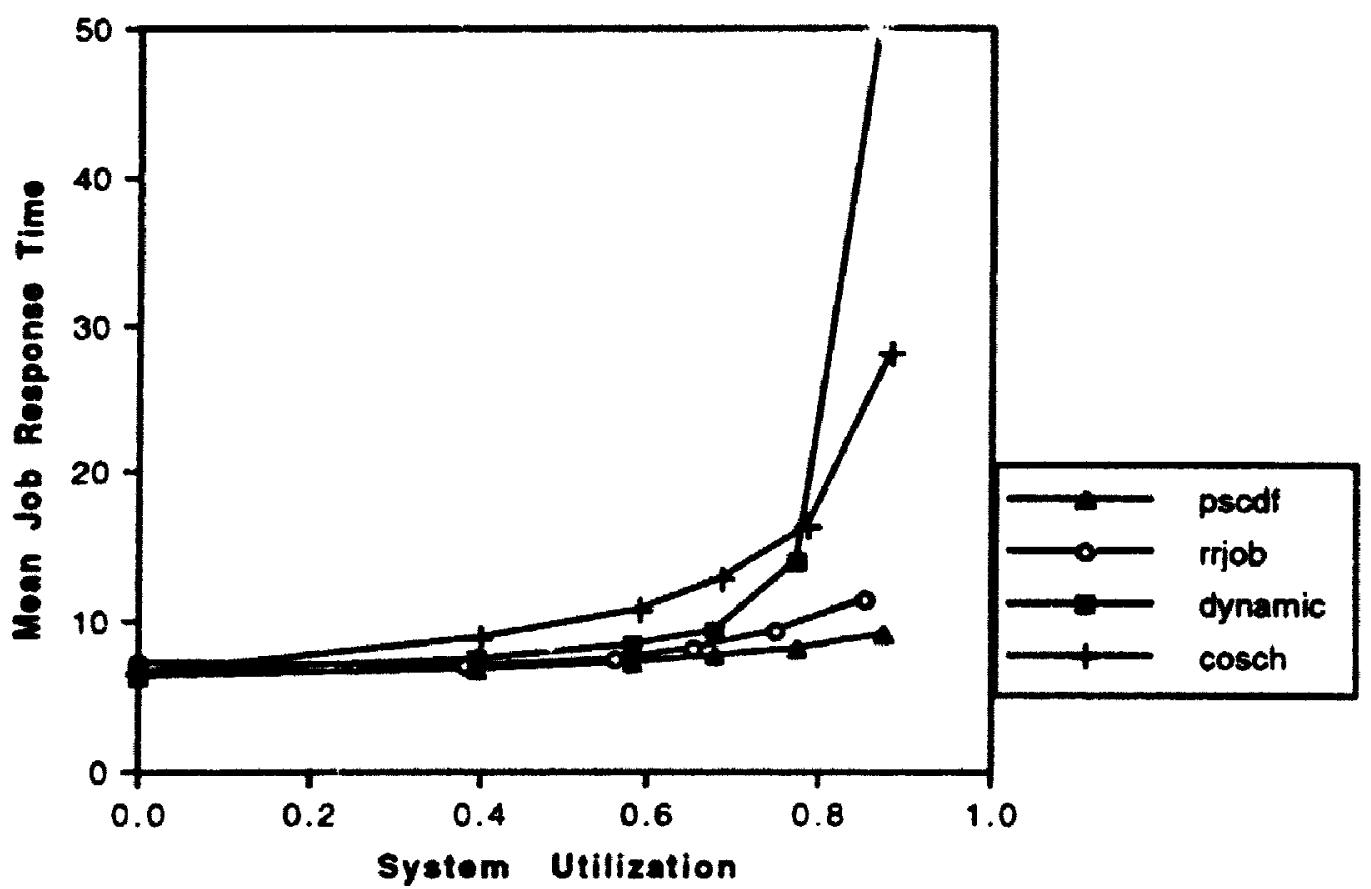

Figure 5.35 Performance of scheduling policies when $C V_{n}=5.0$ (Cor,DC)

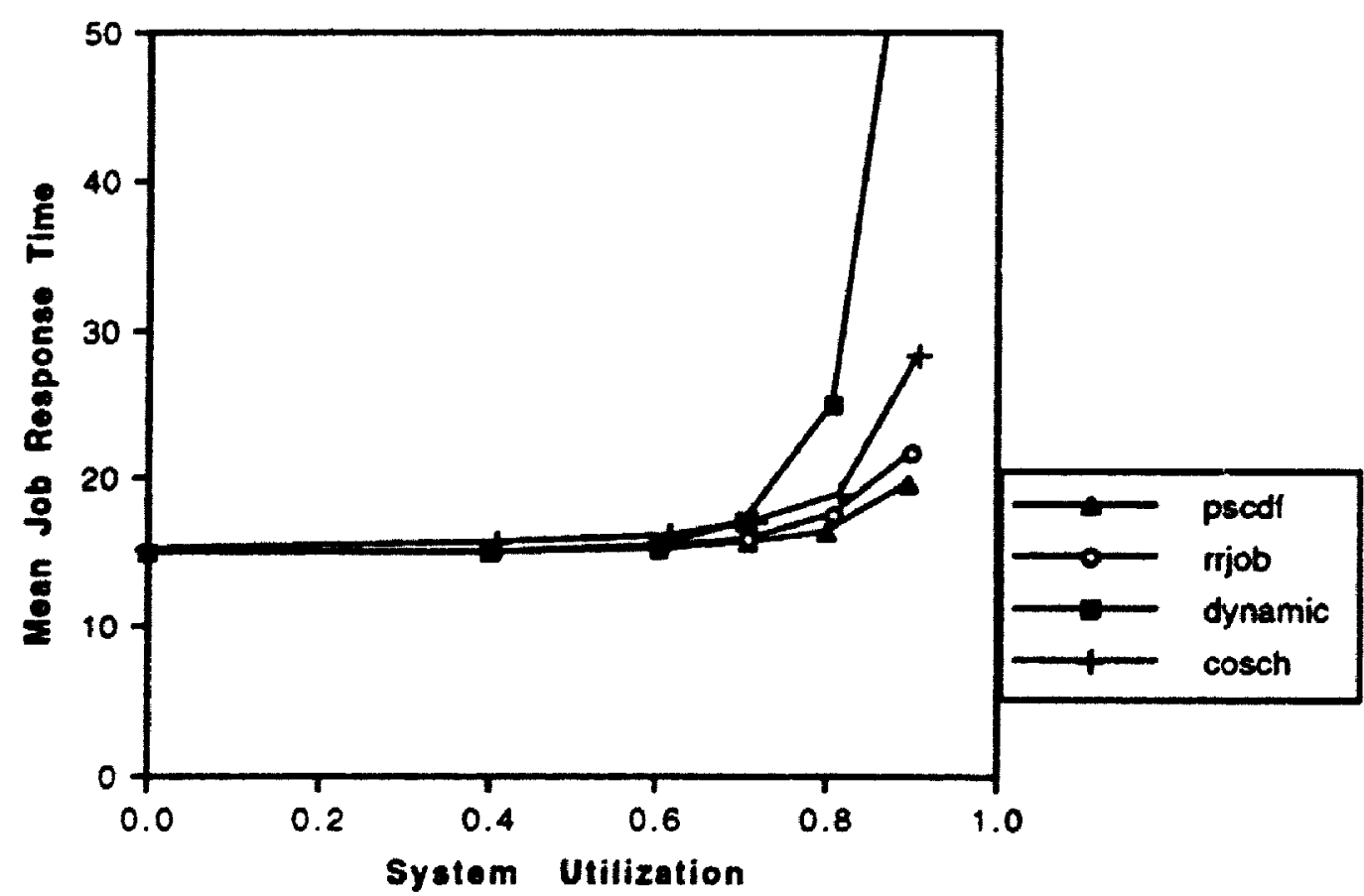

Figure 5.36 Performance of scheduling policies when $C V_{n}=5.0$ (UnCor,DC) 
The results of the correlated and uncorrelated versions of the divide-and-conquer workload model with $C V_{n}=5.0$ is shown in Figures 5.35 and 5.36.

When $C V_{n}=5.0$, there are $28.6718 \%$ of sequential jobs, $58.8366 \%$ of jobs with 1 to 10 tasks, $7.4066 \%$ of jobs with 11 to 20 tasks and $5.085 \%$ of jobs with more than 20 tasks.

Coscheduling performs poorly under the correlated workload. The disproportionate share of the system for the large jobs is the reason for its poor performance. Dynamic performs poorly at high utilization level because the number of jobs in the system often exceeds the number of processors.

When the workload is uncorrelated, the performance of the coscheduling is closer to that of the mjob for the reasons discussed in the last section.

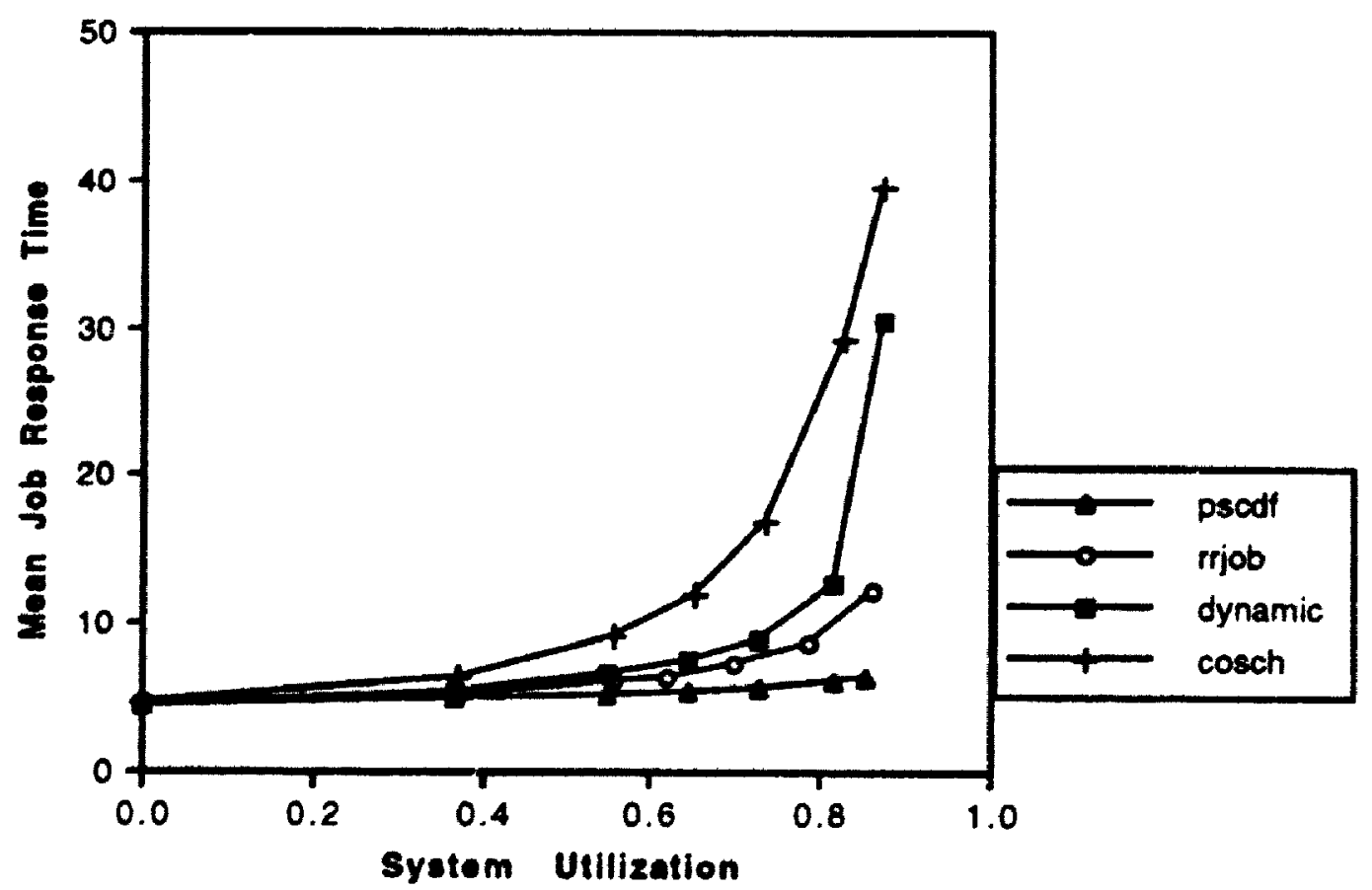

Figure 5.37 Performance of scheduling policies when $C V_{n}=5.0$ (Cor,GE) 


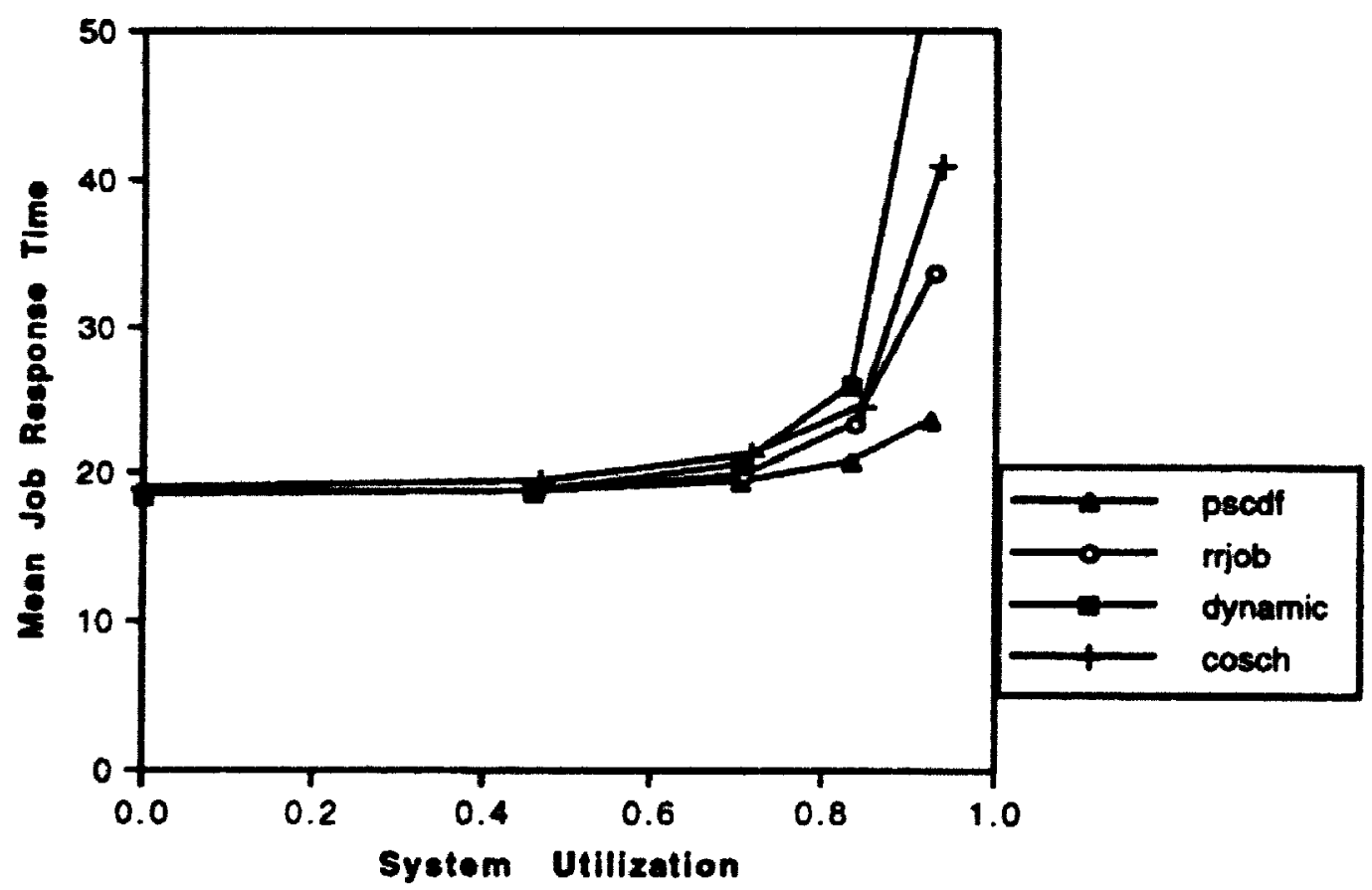

Figure 5.38 Performance of scheduling policies when $C V_{n}=5.0$ (UnCor,GE)

Figures 5.37 and 5.38 plot the results of the correlated and uncorrelated versions of the gaussian elimination workload model. The relative performance of the policies under this workload model is similar to that in the fork-and-join model. 


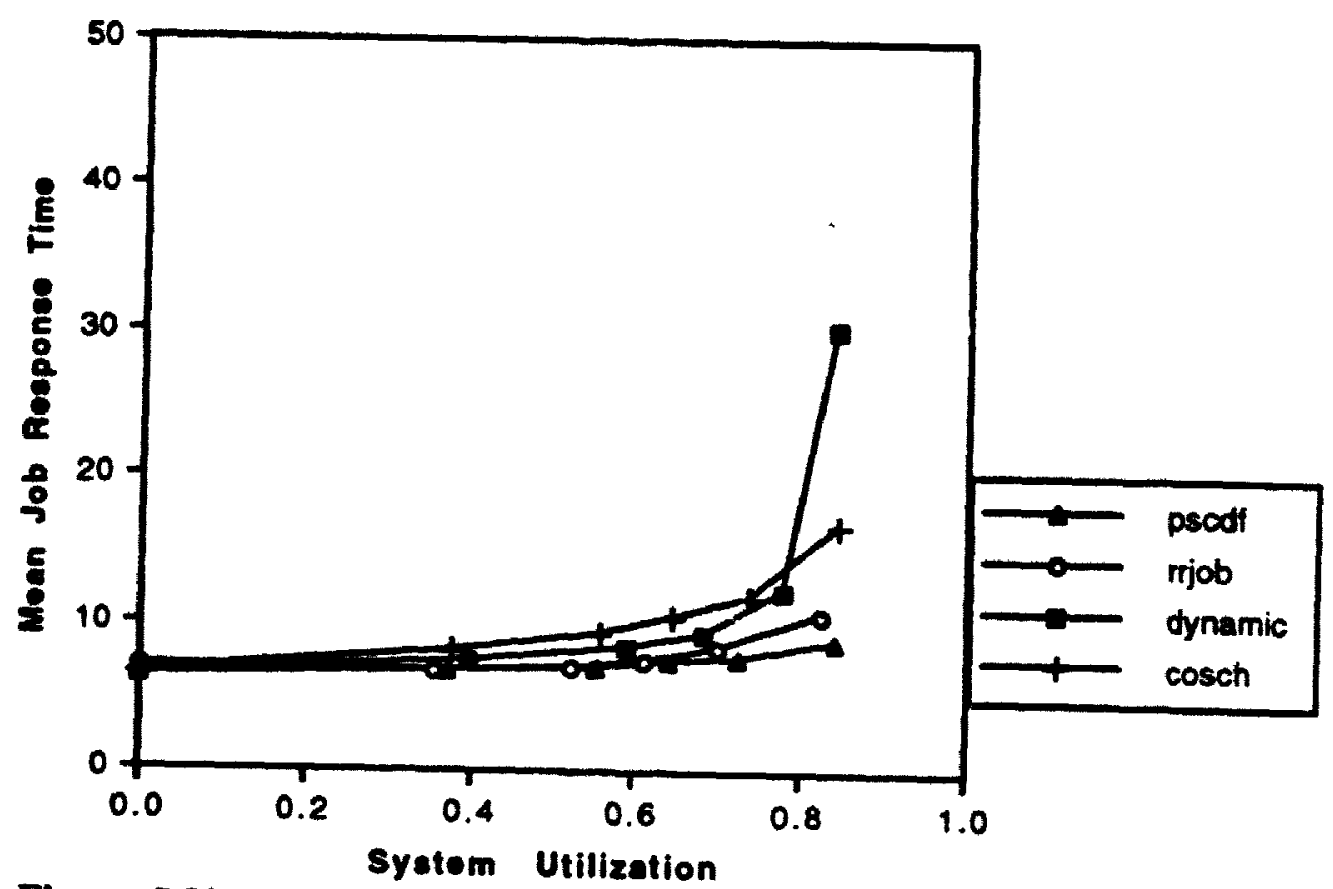

Figure 5.39 Performance of scheduling policies when $C V_{n}=5.0$ (Cor,SSS)

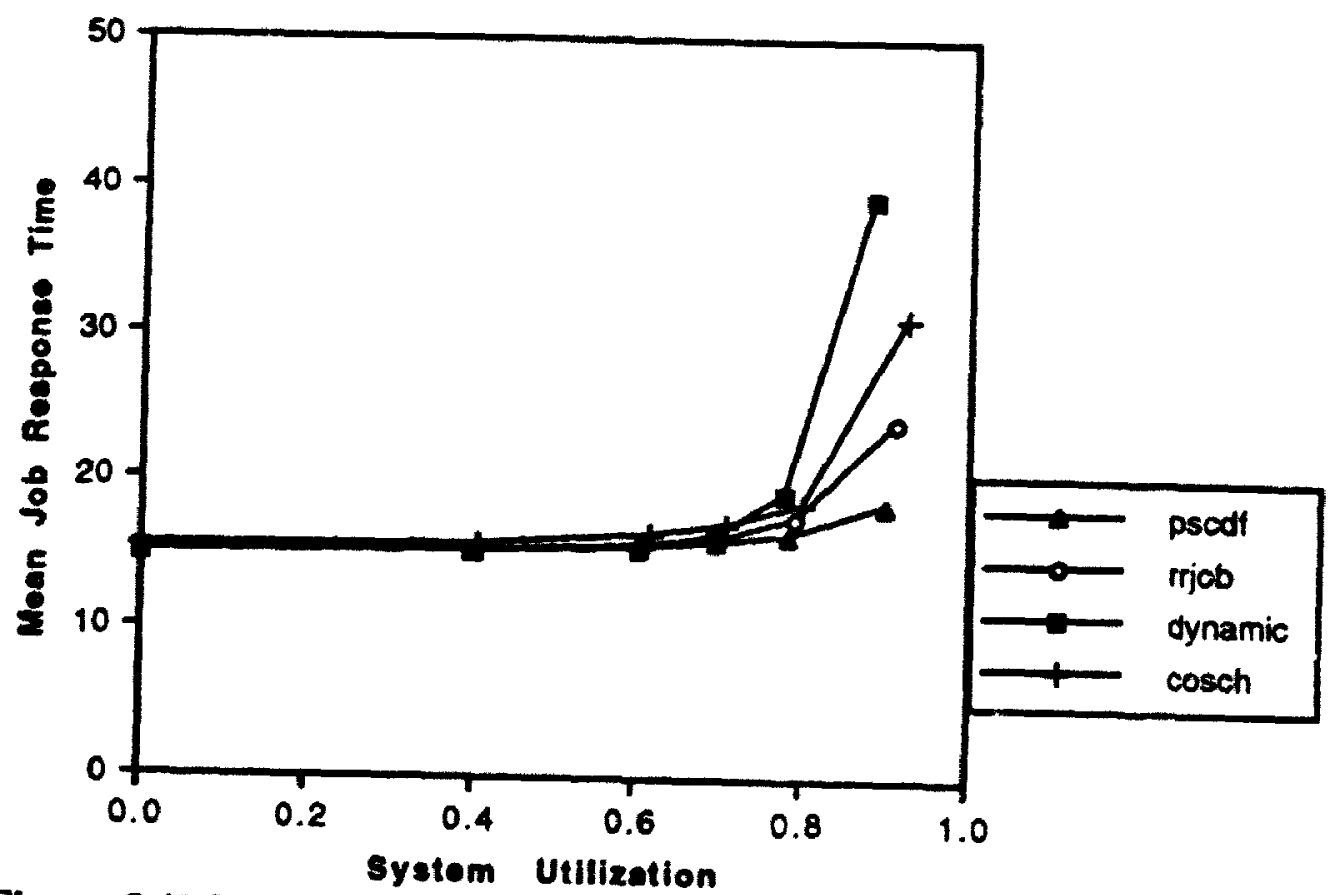

Figure 5.40 Performance of scheduling policies when $C V_{n}=5.0$ (UnCor,SSS)

Figures 5.39 and 5.40 present the results for the correlated and uncorrelated version of the state-space search job model. The relative performance of the policies under this 
workload model is similar to that in the divide-and-conquer job model. Once again it shows that the presence of task abortion does not change the relative performance of the four policies.

The following summarizes that impact of $C V_{n}$ on the performance of the four scheduling policies. For the fork-and-join model, changing the $C V_{n}$ value effects the relative performance. Note that $C V_{n}=0.0$ is a special case where all jobs in the system have the same number of tasks and is not used for the comparison. However, the relative performance of the policies for $C V_{n}=1.0$ and $C V_{n}=5.0$ can be compared.

The coscheduling policy under the fork-and-join workload model is considered first. The performance of coschertuling is close to that of the dynamic policy when $C V_{n}=1.0$. However, it performs worse than the dynamic policy when $C V_{n}=5.0$. This shows that the coscheduling policy is sensitive to variance in job parallelism. Coscheduling gives consecutive quanta to large jobs (in terms of service demand and number of tasks). This results in a disproportionate share of the pr xessing power and longer mean job response time. In general, the relative performance of the policies is not changed by varying the $C V_{n}$ value. This can be seen by comparing the Figures 5.1 and 5.2 to Figures 5.33 and 5.34 .

It can be seen by comparing Figures 5.3 and 5.4 to Figures 5.35 and 5.36 that the relative performance of the four policies is not significantly affected by $C V_{n}$. Except when $C V_{n}=5.0$ and at high utilization levels, the dynamic policy performs worse than the coscheduling. Since dynamic does not time-share the processing power among the jobs, at high utilization levels, parallel jobs need to wait on the queue for a longer time. This results in increased mean job response times.

For the gaussian elimination workload model, changing the $C V_{n}$ values does not affect the relative performance of the policies. This can be seen by comparing Figures 5.5 
and 5.6 to Figures 5.35 and 5.36. Finally, for the state-space search workload model, varying the $C V_{n}$ values has no effect on the relative performance of the policies as well. This can be seen by comparing Figures 5.7 and 5.8 to Figures 5.37 and 5.38 .

\subsection{Conclusions}

From the above experiments and discussions, we have reached some conclusions. They are listed as follows.

- Varying the job structure has significant impact on the performance of the dynamic policy

- Dynamic policy is sensitive to $C V_{d}$ for the fork-and-join model but not for the other models

- Performance of the scheduling policies is not be affected by task-abortion

- Most of the experiments indicate that coscheduling periorms worse than the other policies except when the job structure is fork-and-join and $C V_{n}=0.0$. In this case, the coscheduling policy performs betuer than the others.

- Varying the $C V_{n}$ values does not have significant effect on the relative performance of the policies.

- rjob performs very well for all the job models

- For DC, GE and SSS job models, the performance of dynamic policy is similar to the rjob 


\section{CHAPTER VI}

\section{CONCLUSION}

\subsection{Summary}

Characterizing parallel programs is an important issue for performance evaluation of multiprocessing systems. The goal of this thesis is to investigates the effects of job structures on the performance of scheduling policies for multiprogrammed multiprocessors.

Chapter 1, we discussed various methods of characterizing parallel programs. Chapter 2 is an introduction to scheduling policies and review of the performance of these policies for a simple fork a simple fork-and-join type workload.

Chapter 3 presented a survey of four classes of algorithms and their parallel implementations. They are classified as 1) fork-and-join, 2) divide-and-conquer, 3) Gaussian Elimination and 4) state-space search. An abstract model is then constructed for evaluating the performance of four scheduling policies.

Chapter 4 described the scheduling policies that are selected for performance evaluation.

Simulation experiments are conducted to evaluate the performance of these policies with different job models and the results are reported in Chapter 5.

\subsection{Contribution of the Thesis}

The main contribution of the thesis is in Chapters 3 and 5. Chapter 3 presented an abstract model of parallel programs that incorporates the structure of four categories of parallel programs. Such an approach has never been attempted before. Chapter 5 presented 
the simulation results that compared the performance of the four scheduling policies. We have found that varying the job models affects the scheduling policies only in some special cases. In a normal circumstance, the effect is very limited. In addition, the results also prove thai task abortion does not have any significant effect of the performance of the scheduling policies.

In summary, the results presented show that varying the job structure does not significantly affect the relative performance of the scheduling policies considered. In addition, the dynamic policy is sensitive to $C V_{d}$ for the fork-and-join model but not so for the other models. Moreover the relative performance of the scheduling policies is not affected by the premature task-abortion. For DC, GE and SSS job models, the performance of the dynamic policy is similar to that of the riob.

\subsection{Further Research}

In this thesis, we introduced an abstract model to characterize parallel programs with regular synchronization pattern. We believe that it can also be used to represent programs that belong to other classes. Further research is needed to investigate the representativeness of this model.

The presence of pivot tasks in the GE job model introduces the need for giving priority to these tasks at the application level. It is interesting to investigate its effect on processor scheduling.

Another research direction is to apply this abstract model to study issues other than scheduling such as task routing in parallel distributed systems as the issue of synchronization should have significant impact on the routing decision of such systems. 


\section{References}

[Ak] 89]

Selim G. Akl, The Design and Analysis of Parallel Algorithms , Prentice Hall, Inc., 1989.

[Andrews 83]

Gregory R. Andrews. "Concepts and notations for concurrent programming", ACM Computing Surveys, Vol. 15, No. 1, Mach 1983, pp.3-43.

[AImasi \& Gottlieb 89]

G. Almasi, and A. Gotulieb. Highly Parallel Computing, Benjamin/Cumming, 1989.

[Barr \& Geigenbaum 81]

Avron Barr and Edward A. Geigenbaum. The Handbook of Artificial Intelligence. Vol.1, William Kaufmann, Inc., 1981.

[Bitton et al. 83]

Dina Bitton, Haran Boral, David J. DeWitt and Kevin Wilkinson, "Parailel algorithms for the execution of relational database operations", ACM Trans. on Database Systems, Vol.8, No.3, Sep.1983, pp. 324-353

[Chandy \& Mirsa 81]

K. M. Chandy, and J. Mirsa, "Asynchronous distributed simulation via a sequence of parallel computations", Communications of the ACM, Vol. 24, No. 11, Arpil 1981. pp. 198-206.

[Deminet 82]

Jarek Deminet, "Experience with multiprocessor algorithms", I.E.E.E. Trans. on Comp., Vol. C-31, No.4, April 1982, pp.278-288.

[Evans \& Yousif 85]

D.J. Evans, Nadia Y. Yousif, "Analysis of the performance of the parallel quicksort method", BIT, Vol. 25, 1985 pp.106-112

[Franklin 78]

Mark A. Franklin, "Parallel solution of ordinary differential equations", I.E.E.E. Trans. on Comp., Vol. C-27, No. 5, May 1978, pp. 413-420. 
[Gallivan \& Plemmons 90]

K.A. Gallivan, R.J. Plemmons, and A.H. Sameh. "Parallel algorithms for dense linear algebra computations", Parallel Algorithms for Matrix Computations, 1990. SIAM, pp.1-82.

[George et al. 86]

Alan George, Michael T. Heath and Joesph Liu. "Parallel Cholesky factorization on a shared-memory multiprocessor", Linear Algebra and Its Applications 77, 1986. pp.165-187.

[Gerasoulis \& Nelken 89]

Apostolos Gerasoulis and Izzy Nelken, "Scheduling linear algebra parallel algorithms on MIMD architectures", Proceedings of The Fourth SIAM Conference on Parallel Processing for Scientific Computing, Dec. 1989

[Ghosal et al. 91]

Dipak Ghosal, Giuseppe Serazzi and Satish K. Tripathi. "The processor working set and its use in scheduling multiprocessor systems". IEEE Transactions on Sofrware Engineering, Vol. 17. No. 5. May 1991.

[Golub \& Van Loan 89]

Gene H. Golub and Charles F. Van Loan, Matrix Computations, The Johns Hopkins University Press, 1989.

[Grit \& Mcgraw 85]

Dale H. Grit and James R. Mcgraw, "Programming divide and conquer for a MMD machine", Sofiware-Practice and Experience, Vol. 15(1), Jan. 1985, pp.41-53

[Gupta et al. 91]

Anoop Gupta, Andrew Tucker, and Shigeru Urushibara, "The impact of operating system scheduling policies and synchronization methods on the performance of parallel applications", Proc. of the 1991 ACM SIGMETRICS Conference on Measurement and Modelling of Computer Systems, May 1991, pp.120-132.

[Hachtel \& Moceyunas 88]

Gary D. Hachtel and Peter H. Moceyunas, "Parallel algorithms for answering the tautology question", I.E.E.E. International Conf. on Para. Proc., 1988, pp. 133-138. 
[Hagan et al. 85]

Martin T. Hagan, Howard B. Demuth, Paul H. Singgih, "Parallel signal processing research on the HEP", Proc. of 1985 International Conference on Parallel Processing. pp.599 - 606.

[Horowitz \& Zorat 83]

Ellis Horowitz and Alessandro Zorat, "Divide-and-conquer for parallel processing". I.E.E.E. Trans. on Comp., Vol. C-32, No. 6, June 1983. pp.582-585

[Horowitz \& Sahni 78]

Ellis Horowitz and Sartaj Sahni, Fundamentals of Computer Algorithms, Computer Science Press, 1978.

[Huang \& Ongsakul 91]

G. Huang, W. Ongsakul, Parallel implementation of gauss-seidel type algorithms for power analysis on a SEQUENT parallel computer", Proc. of IEEE Int. Conf. on Para. Proc., Vol. III, 1991, pp.171-174.

[Knecht 89]

Renate Knecht, "Implementation of divide-and-conquer algorithms on multiprocessors". Proceedings of WOPPLOT 89, pp.121-136.

[Knecht 90]

Renate Knecht, "Parallelizing divide-and-conquer algorithms microtasking versus autotasking", Joint International Conference on Vector and Parallel Processing, Zurich, Switzerland, Sep. 1990, pp. 548-557.

[Kobayashi 81]

Hisashi Kobayashi, Modeling and Analysis: An Introduction to System Performance Evaluation Methodology, October 1981. Addison-Wesley Publishing Co.

[Kumar \& Kowalik 84]

Swarn P. Kumar and Janusz S. Kowalik, "Parallel factorization of positive definite matrix on an MIMD computer", Proc. of the 1984 International Conf. on Parallel Processing, pp.410-416. 
[Kung 76]

H. T. Kung, "Synchronized and asynchronous parallel algorithms", in J. F. Traub, Ed., Algorithms and Complexity: New Directions and Recent Results, Academic, New York, 1976, pp. 153-200.

[Lenoski et al. 92]

Daniel Lenoski, James Laudon, Kourosh Gharachorloo, Wolf-Dietrich Weber, Anoop Gupta, John Hennessy, Mark Horowitz, and Monica S. Lam "The Stanford Dash multiprocessor", IEEE Computer, Vol. 25, No. 3, March 1992, pp.63-79.

[Leutenegger 90]

Scott T. Leutenegger. "Issues in multiprogrammed multiprocessor scheduling", Computer Sciences Technical Report \#954, Computer Sciences Dept., University of Wisconsin-Madison, Aug. 1990.

[Leutenegger \& Vernon 90]

Scott T. Leutenegger, and Mary K. Vernon. "The performance of multiprogrammed multiprocessor scheduling policies", Proc. of 1990 ACM SIGMETRICS Conference on Measurement and Modelling of Computer Systems, pp.226 - 236.

[Leutenegger \& Vernon 92]

Scott T. Leutenegger, and Mary K. Vemon, "Multiprogrammed multiprocessor scheduling issues", IBM Research Report \#77799, Feb. 1992.

[Lovett \& Thakker 88]

T. Lovett, and S. Thakker, "The Symmetry multiprocessor system", Proceedings of the 1988 Intemational Conference on Parallel Processing, August, 1988.

[Lord et al. 83]

Lord, R.E., Kowalik, J.S. and Kumar, S.P., Solving Linear A.igebraic Equations on an MMD Computer, Jounral of the ACM, Vol. 30, 1983, pp.103-117.

[Madala \& Sinclair 91]

Sridhar Madala and James B. Sinclair. "Performance of synchronous parallel algorithms with regular structures", IEEE Transactions on Parallel and Distributed Systems, Vol. 2, No. 1, Jan. 1991. pp.105-116. 
[Majumdar et al. 88]

Shikharesh Majumdar, Derek L. Eager and Richard B. Bunt, "Scheduling in multiprogrammed paraiiei systcms", Proc. of 1988 ACM SIGMETRICS Conference on Measurement and Modelling of Computer Systems, pp.104-113.

[Majumdar et al. 91]

Shikharesh Majumdar, Derek L. Eager and Richard B. Bunt, "Characterisation of programs for scheduling in multiprogrammed parallel systems". Performance Evaluation, Vol. 13, 1991, pp.109-130.

[McCann et al. 90]

Cathy McCann, Raj Vaswani, and John Zahorjan, “A dynamic processor allocation policy for multiprogrammed, shared memory multiprocessor", Technical Report 90-0302. Dept. of Comp. Sci. and Eng., University of Washington, March 1990.

[Natarajan 89]

K. S. Natarajan, "Expected Performance of Parallel Search", Proc. of the 1989 Iniernational Conference on Parallel Processing, Vol.3, Aug. 1989, pp.121-125.

[Preparata \& Hong 77]

F. P. Preparata and S. J. Hong, "Convex hulls of finite sets of points in two and three dimensions", Communicarions of the ACM, Feb. 1977, pp. 87 - 93.

[Ostlund et al. 82]

Neil S. Ostlund, Peter G. Hibbard and Robert A. Whitside, "A case study in the application of a ightiy coupled multiprocessor to scientific computations", in Garry Rodrigue, Ed., Parallel Computations, Academic Press, New York. 1982, pp.315364.

[Ousterhout 82]

John K. Ousterhout. "Schedsling techni ues for concurrent systems", Proc. of 3rd Intemational Conference on Distributed Computing Systems , October 1982, p.22-30

[Rose 9(1)

Jonathan Rose, "Parallel global routing for standard cells", IEEE Transactions on Computer-Aided Design, Vol. 9, No. 10, October 1990, pp.1085-1095. 
[Sevcik 89]

Kenneth C. Sevcik, "Characterizations of parallelism in applications and their use in scheduling", Performance Evaluation Review, Vol. 17, No. 1, May 1989, pp.171180.

[Shekhawat \& Chakravarty 90]

Ajay Shekhawat, and Sreejit Chakravarty. "Heuristics for the MSC problem for serial and shared-memory computers". Proc. of the 1990 International Conf. on Parallel Processing, Vol.3, Aug. 1990, pp.64-67.

[Singh et al. 91]

Jaswinder Pal Singh, Wolf-Dietrich Weber and Anoop Gupta, "SPLASH: Stanford parallel applications for shared-memory", Technical Report CSL-TR-91-469, Computer Systems Lab., Stanford University, 1991.

[Simth 78]

B. J. Simth, "A pipelined, shared resource MIMD computer", Proc. of the 1978 Int. Conf. on Parallel Processing, Aug. 1978, pp.6-8.

[Stewart 73]

G. W. Stewart, Introduction to Matrix Computations, 1973, Academic Press, Inc.

[Tucker \& Gupta 89]

Andrew Tucker and Anoop Gupta, "Prscess Control and Scheduling issues for multiprogrammed shared-memory multiprocessors", Proc. of the 12th ACM Symposium on Operating Systems Principles, Dec. 1989, pp. 159-166.

[Zahorjan et al. 91]

John Zahorjan, Edward D. Lazowska and Derek L. Eager, "The effect of scheduling discipline on spin overhead in shared memory parallel systems". IEEE Transactions on Parallel and Distributed Systems, vol. 2, no. 2, April 1991, pp.180-198.

[Zahorjan \& McCann 90]

John Zahorjan and, Cathy McCann. "Processor scheduling in shared memory multiprocessors", Proc. of 1990 ACM SIGMETRICS Conference on Measurement and Modelling of Computer Systems, pp.214-225 

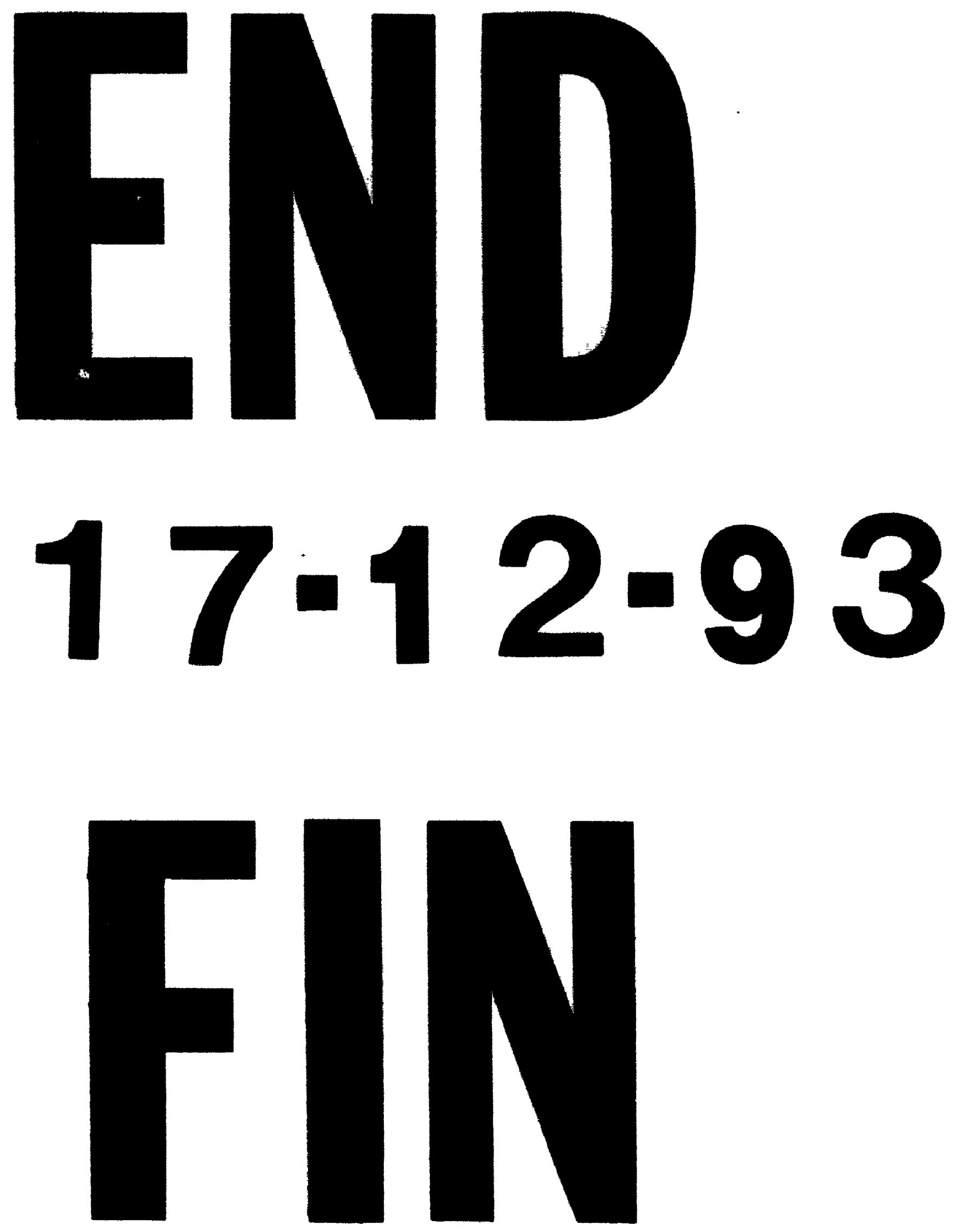